\title{
NILPOTENT BICONE AND CHARACTERISTIC SUBMODULE OF A REDUCTIVE LIE ALGEBRA.
}

\author{
by \\ Jean-Yves Charbonnel \& Anne Moreau
}

\begin{abstract}
Let $\mathfrak{g}$ be a finite dimensional complex reductive Lie algebra and $\mathrm{S}(\mathfrak{g})$ its symmetric algebra. The nilpotent bicone of $\mathfrak{g}$ is the subset of elements $(x, y)$ of $\mathfrak{g} \times \mathfrak{g}$ whose subspace generated by $x$ and $y$ is contained in the nilpotent cone. The nilpotent bicone is naturally endowed with a scheme structure, as nullvariety of the augmentation ideal of the subalgebra of $S(\mathfrak{g}) \otimes_{\mathbb{C}} S(\mathfrak{g})$ generated by the 2 -order polarizations of invariants of $S(\mathfrak{g})$. The main result of this note is that the nilpotent bicone is a complete intersection of dimension $3\left(b_{\mathfrak{g}}-\mathrm{rkg}\right)$, where $\mathrm{b}_{\mathfrak{g}}$ and $\mathrm{rkg}$ are the dimension of Borel subalgebras and the rank of $\mathfrak{g}$ respectively. This affirmatively answers a conjecture of KraftWallach concerning the nullcone $\mathbf{K r W 2}$. In addition, we introduce and study in this note the characteristic submodule of $\mathfrak{g}$. The properties of the nilpotent bicone and the characteristic submodule are known to be very important for the understanding of the commuting variety and its ideal of definition. The main difficulty encountered for this work is that the nilpotent bicone is not reduced. To deal with this problem, we introduce an auxiliary reduced variety, the principal bicone. The nilpotent bicone, as well as the principal bicone, are linked to jet schemes. We study their dimensions using arguments from motivic integration. Namely, we follow methods developed by M. Mustaţă in $\mathbf{M u}$. At last, we give applications of our results to invariant theory.
\end{abstract}

\section{Contents}

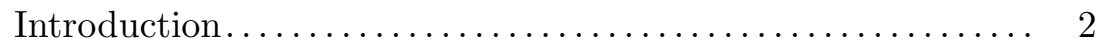

1. Characteristic submodule.......................

2. Principal cone and principal bicone.................. 13

3. Jet schemes and motivic integration................ 20

4. Dimension of the nilpotent bicone via motivic integration . 33

5. Applications to invariant theory.................. 39

6. Additional properties of the nilpotent bicone............ 41

References..................................... 46

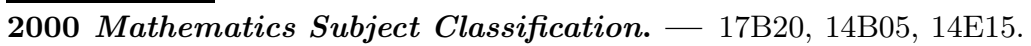

Key words and phrases. — nilpotent cone, nilpotent bicone, polarizations, nullcone, rational singularities, jet scheme, motivic integration. 


\section{Introduction}

1. Main results. - Let $\mathfrak{g}$ be a finite dimensional complex reductive Lie algebra, let $G$ be its adjoint group and let $\mathfrak{N}_{\mathfrak{g}}$ be its nilpotent cone. For $(x, y)$ in $\mathfrak{g} \times \mathfrak{g}$, we denote by $P_{x, y}$ the subspace generated by $x$ and $y$. A subset of $\mathfrak{g} \times \mathfrak{g}$ is called bicone if it is stable under the maps $(x, y) \mapsto(s x, t y)$ where $s$ and $t$ are in $\mathbb{C}^{*}$. The nilpotent bicone of $\mathfrak{g}$ is the subset

$$
\mathcal{N}_{\mathfrak{g}}:=\left\{(x, y) \in \mathfrak{g} \times \mathfrak{g} \mid P_{x, y} \subset \mathfrak{N}_{\mathfrak{g}}\right\} .
$$

Since $\mathfrak{N}_{\mathfrak{g}}$ is a $G$-invariant closed cone of $\mathfrak{g}$, the subset $\mathcal{N}_{\mathfrak{g}}$ is a closed bicone of $\mathfrak{g} \times \mathfrak{g}$, invariant under the diagonal action of $G$ on $\mathfrak{g} \times \mathfrak{g}$.

Let $\mathrm{S}(\mathfrak{g})$ be the symmetric algebra of $\mathfrak{g}$ and let $\mathrm{S}(\mathfrak{g})^{\mathfrak{g}}$ be the subalgebra of $G$ invariant elements of $\mathrm{S}(\mathfrak{g})$. According to a Chevalley's result, the algebra $S(\mathfrak{g})^{\mathfrak{g}}$ is polynomial in $\mathrm{rkg}$ variables, where $\mathrm{rk} \mathfrak{g}$ is the rank of $\mathfrak{g}$. Let $p_{1}, \ldots, p_{\text {rkg }}$ be homogeneous generators of $\mathrm{S}(\mathfrak{g})^{\mathfrak{g}}$ of degrees $d_{1}, \ldots, d_{\mathrm{rkg}}$ respectively. We can suppose that the sequence $d_{1}, \ldots, d_{\mathrm{rkg}}$ is weakly increasing. For $i=1, \ldots, \mathrm{rk} \mathfrak{g}$, the 2 -order polarizations $p_{i, m, n}$ of $p_{i}$ are the unique elements of $\left(\mathrm{S}(\mathfrak{g}) \otimes_{\mathbb{C}} \mathrm{S}(\mathfrak{g})\right)^{\mathfrak{g}}$ satisfying the following relation:

$$
p_{i}(a x+b y)=\sum_{m+n=d_{i}} a^{m} b^{n} p_{i, m, n}(x, y),
$$

for all $(a, b)$ in $\mathbb{C}^{2}$ and $(x, y)$ in $\mathfrak{g} \times \mathfrak{g}$. The nilpotent cone $\mathfrak{N}_{\mathfrak{g}}$ is the nullvariety in $\mathfrak{g}$ of the ideal generated by the polynomials $p_{1}, \ldots, p_{\mathrm{rkg}}$. Therefore, according to Relation (11), $\mathcal{N}_{\mathfrak{g}}$ is the nullvariety in $\mathfrak{g} \times \mathfrak{g}$ of the ideal of $\mathrm{S}(\mathfrak{g}) \otimes_{\mathbb{C}} \mathrm{S}(\mathfrak{g})$ generated by the polynomials $p_{i, m, n}$, for $i=1, \ldots$, rk $\mathfrak{g}$ and $m+n=d_{i}$. Thus, $\mathcal{N}_{\mathfrak{g}}$ is naturally endowed with a scheme structure. From here, we study $\mathcal{N}_{\mathfrak{g}}$ as the subscheme of $\mathfrak{g} \times \mathfrak{g}$ corresponding to the ideal generated by the polynomials $p_{i, m, n}$, for $i=1, \ldots$, rk $\mathfrak{g}$ and $m+n=d_{i}$.

Let $b_{\mathfrak{g}}$ be the dimension of a Borel subalgebra of $\mathfrak{g}$. By a classical result Bou, we have $d_{1}+\cdots+d_{\text {rkg }}=b_{\mathfrak{g}}$. So $\mathcal{N}_{\mathfrak{g}}$ is the nullvariety in $\mathfrak{g} \times \mathfrak{g}$ of $b_{\mathfrak{g}}+\mathrm{rk} \mathfrak{g}$ polynomial functions. As a result, the dimension of any irreducible component of $\mathcal{N}_{\mathfrak{g}}$ is at least $3\left(\mathrm{~b}_{\mathfrak{g}}-\mathrm{rk} \mathfrak{g}\right)$, since $\mathfrak{g} \times \mathfrak{g}$ has dimension $2\left(2 \mathrm{~b}_{\mathfrak{g}}-\mathrm{rk} \mathfrak{g}\right)$. The main result of this note is the following theorem (see Theorem 33, (iv), Proposition 36. (ii), and Theorem 45):

Theorem 1. - The nilpotent bicone is a nonreduced complete intersection in $\mathfrak{g} \times \mathfrak{g}$ of dimension $3\left(\mathrm{~b}_{\mathfrak{g}}-\mathrm{rk} \mathfrak{g}\right)$. Moreover, the images of any irreducible component of $\mathcal{N}_{\mathfrak{g}}$ by the first and second projections from $\mathfrak{g} \times \mathfrak{g}$ to $\mathfrak{g}$ are equal to $\mathfrak{N}_{\mathfrak{g}}$.

This result affirmatively answers a conjecture KrW2 (Section 1) of H. Kraft and N. Wallach concerning the nullcone of the $G$-module $\mathfrak{g} \times \mathfrak{g}$ (see Theorem 41). Clearly, the images of $\mathcal{N}_{\mathfrak{g}}$ by the first and second projections 
from $\mathfrak{g} \times \mathfrak{g}$ to $\mathfrak{g}$ are equal to $\mathfrak{N}_{\mathfrak{g}}$. Theorem 1 specifies that this is true for any irreducible component of $\mathcal{N}_{\mathfrak{g}}$. Notice that this statement is a much stronger result.

We introduce in addition in this note the characteristic submodule of $\mathfrak{g}$. It is a $S(\mathfrak{g}) \otimes_{\mathbb{C}} S(\mathfrak{g})$-submodule of $S(\mathfrak{g}) \otimes_{\mathbb{C}} S(\mathfrak{g}) \otimes_{\mathbb{C}} \mathfrak{g}$. The properties of the nilpotent bicone and the characteristic submodule are known to be very important for the understanding of the commuting variety. Recall that the commuting variety $\mathcal{C}_{\mathfrak{g}}$ of $\mathfrak{g}$ is the set of elements $(x, y)$ of $\mathfrak{g} \times \mathfrak{g}$ such that $[x, y]=0$. The commuting variety has been studied for many years. According to a result of R. W. Richardson Ri1, $\mathcal{C}_{\mathfrak{g}}$ is irreducible. Moreover, $\mathcal{C}_{\mathfrak{g}}$ is the nullvariety of the ideal generated by the elements $(x, y) \mapsto\langle v,[x, y]\rangle$, where $v$ runs through $\mathfrak{g}$. An old unsolved question is to know whether this ideal is prime [LS]. In other words, we want to know if this ideal is the ideal of definition of $\mathcal{C}_{\mathfrak{g}}$ since $\mathcal{C}_{\mathfrak{g}}$ is irreducible. The study of the commuting variety and of its ideal of definition is a main motivation for our work.

2. Description of the paper. - We denote by $\mathfrak{g}_{\mathrm{reg}}$ the subset of regular elements of $\mathfrak{g}$ and we set

$$
\Omega_{\mathfrak{g}}:=\left\{(x, y) \in \mathfrak{g} \times \mathfrak{g} \mid P_{x, y} \backslash\{0\} \subset \mathfrak{g}_{\mathrm{reg}}, \operatorname{dim} P_{x, y}=2\right\} .
$$

The properties of the subset $\Omega_{\mathfrak{g}}$ were studied by A. V. Bolsinov in [Bol] and recently by D. I. Panyushev and O. Yakimova in PYa. The set of smooth points of $\mathcal{N}_{\mathfrak{g}}$ with respect to its scheme structure is the set of elements $(x, y) \in$ $\mathfrak{g} \times \mathfrak{g}$ at which the differentials of the $p_{i, m, n}$, for $i=1, \ldots, \mathrm{rkg}$ and $m+n=$ $d_{i}$, are linearly independent. The set $\Omega_{\mathfrak{g}}$ is an open subset of $\mathfrak{g} \times \mathfrak{g}$ and its intersection with the nilpotent bicone turns out to be the set of smooth points of $\mathcal{N}_{\mathfrak{g}}$ (see Proposition 13 and Remark 3 ). The description of this subset is therefore very important for us. We give various properties of the subset $\Omega_{\mathfrak{g}}$ in Section 1. Next, we introduce in this section the characteristic submodule $\mathrm{B}_{\mathfrak{g}}$ of $\mathfrak{g}$ (see Definition 1) whose study is heavily related to $\Omega_{\mathfrak{g}}$. The characteristic submodule was introduced by the first author a few years ago in a work on the commuting variety. We prove here that $B_{\mathfrak{g}}$ is a free $S(\mathfrak{g}) \otimes_{\mathbb{C}} S(\mathfrak{g})$-module of rank $b_{\mathfrak{g}}$, and we provide an explicit basis for $B_{\mathfrak{g}}$ (see Theorem 11). Even if this result is not directly useful for the nilpotent bicone, it could be interesting in itself.

The results obtained in Section 1 concerning $\Omega_{\mathfrak{g}}$ do not enable to provide "enough" smooth points of $\mathcal{N}_{\mathfrak{g}}$ to apply the criterion of Kostant [Kos2] (see Remark 3). Actually, the intersection of $\mathcal{N}_{\mathfrak{g}}$ and $\Omega_{\mathfrak{g}}$ is an empty set in many cases. This observation makes the study of the scheme $\mathcal{N}_{\mathfrak{g}}$ very difficult. In order to deal with this problem, we introduce an auxiliary reduced scheme, the principal bicone of $\mathfrak{g}$

$$
\mathcal{X}_{\mathfrak{g}}:=\left\{(x, y) \in \mathfrak{g} \times \mathfrak{g} \mid P_{x, y} \subset \mathfrak{X}_{\mathfrak{g}}\right\},
$$


where $\mathfrak{X}_{\mathfrak{g}}$ is the principal cone of $\mathfrak{g}$, that is the Zariski closure of the set of principal semisimple elements (see Definitions 2 and 3 ). The study of the varieties $\mathfrak{X}_{\mathfrak{g}}$ and $\mathcal{X}_{\mathfrak{g}}$ is the main topic of Section 2 .

We observe that $\mathcal{N}_{\mathfrak{g}}$ and $\mathcal{X}_{\mathfrak{g}}$ can be identified with constructible subsets of jet schemes of $\mathfrak{N}_{\mathfrak{g}}$ and $\mathfrak{X}_{\mathfrak{g}}$ respectively. In [Mu], M. Mustaţă uses the theory of motivic integration, as developed by M. Kontsevich [Kon, J. Denef and F. Loeser [DL2], and V. Batyrev [Ba], to prove a result concerning the jet schemes of locally complete intersections. In particular, his result can be applied to $\mathfrak{N}_{\mathfrak{g}}$ and $\mathfrak{X}_{\mathfrak{g}}$. Thus, in the appendix of $[\mathbf{M} \mathbf{u}]$, D. Eisenbud and E. Frenkel extend results of Kostant concerning the nilpotent cone of a reductive Lie algebra in the setting of jet schemes. In Section 3, after reviewing some facts about motivic integration, we state technical results concerning motivic integrals, following methods developed by M. Mustaţă in $[\mathbf{M u}$, which will be useful for Section 4. Using all of this, we prove in Section 4 the following theorem (see Theorem 33, (i), and Proposition 36, (i)):

Theorem 2. - The principal bicone of $\mathfrak{g}$ is a reduced complete intersection of dimension $3\left(b_{\mathfrak{g}}-\mathrm{rkg}+1\right)$. Moreover, the images of any irreducible component of $\mathcal{X}_{\mathfrak{g}}$ by the first and second projection from $\mathfrak{g} \times \mathfrak{g}$ to $\mathfrak{g}$ are equal to the principal cone $\mathfrak{X}_{\mathfrak{g}}$.

Then, we deduce Theorem 1 from Theorem 2, We give in Section 5 applications of our results to invariant theory, mainly in relation with the nullcone of the $G$-module $\mathfrak{g} \times \mathfrak{g}$. In Section 6 , we obtain additional properties about the irreducible components of the nilpotent bicone. We prove that $\mathcal{N}_{\mathfrak{g}}$ is a nonreduced scheme (see Theorem 45) and we give a lower bound for the number of irreducible components of $\mathcal{N}_{\mathfrak{g}}$ (see Proposition [52).

We precise that Section 3 is entirely independent on Sections 1 and 2 and does not deal with Lie algebras.

3. Additional notations and conventions. - In this note, the ground field is $\mathbb{C}$. All topological terms refer to the Zariski topology. If $X$ is an algebraic variety, an open subset of $X$ whose complementary in $X$ has codimension at least 2 is called a big open subset of $X$.

The set of integers, the set of nonnegative integers and the set of positive integers are denoted by $\mathbb{Z}, \mathbb{N}$ and $\mathbb{N}^{*}$ respectively. As usual, the subset of non zero elements of $\mathbb{C}$ is denoted by $\mathbb{C}^{*}$.

If $E$ is a finite set, its cardinality is denoted by $|E|$. 
For $x$ in $\mathfrak{g}$, we denote by $\mathfrak{g}(x)$ the centralizer of $x$ in $\mathfrak{g}$. Thus $x$ belongs to $\mathfrak{g}_{\text {reg }}$ if and only if $\mathfrak{g}(x)$ has dimension rkg.

The action of $G$ in $\mathfrak{g} \times \mathfrak{g}$ will be always the diagonal action. For $x$ in $\mathfrak{g}$ or in $\mathfrak{g} \times \mathfrak{g}$, we denote by $G . x$ its $G$-orbit under the corresponding $G$-action.

If $\mathfrak{g}$ is commutative, then $\mathcal{N}_{\mathfrak{g}}$ is reduced to $\{(0,0)\}$. Henceforth, we suppose that $\mathfrak{g}$ is not commutative.

Let $\mathfrak{g}_{1}, \ldots, \mathfrak{g}_{m}$ be the simple factors of $\mathfrak{g}$. Since the nilpotent cone $\mathfrak{N}_{\mathfrak{g}}$ is the product of the nilpotent cones $\mathfrak{N}_{\mathfrak{g}_{1}}, \ldots, \mathfrak{N}_{\mathfrak{g} m}, \mathcal{N}_{\mathfrak{g}}$ is the product of the nilpotent bicones $\mathcal{N}_{\mathfrak{g}_{1}}, \ldots, \mathcal{N}_{\mathfrak{g}_{m}}$. Furthermore, as the equality

$$
3\left(\mathrm{~b}_{\mathfrak{g}}-\mathrm{rk} \mathfrak{g}\right)=3\left(\mathrm{~b}_{\mathfrak{g}_{1}}-\mathrm{rk}_{\mathfrak{g}_{1}}\right)+\cdots+3\left(\mathrm{~b}_{\mathfrak{g}_{m}}-\mathrm{rk} \mathfrak{g}_{m}\right)
$$

holds, it suffices to prove Theorem 1 in the case where $\mathfrak{g}$ is simple. It will be sometimes useful to make the assumption that $\mathfrak{g}$ is simple (see e.g. Sections 2. 5 and 6).

The first and second projections from $\mathfrak{g} \times \mathfrak{g}$ to $\mathfrak{g}$ are denoted by $\varpi_{1}$ and $\varpi_{2}$ respectively. The following lemma will be very handy throughout the note.

Lemma 3. - Let $Y$ be a $G$-invariant closed bicone in $\mathfrak{g} \times \mathfrak{g}$. Then the subsets $\varpi_{1}(Y)$ and $\varpi_{2}(Y)$ are $G$-invariant closed cones of $\mathfrak{g}$.

Proof. - As $Y$ is a $G$-invariant bicone, $\varpi_{1}(Y)$ and $\varpi_{2}(Y)$ are $G$-invariant cones of $\mathfrak{g}$. Moreover, $\varpi_{1}(Y) \times\{0\}$ and $\{0\} \times \varpi_{2}(Y)$ are the intersections of $Y$ with $\mathfrak{g} \times\{0\}$ and $\{0\} \times \mathfrak{g}$ respectively, since $Y$ is a closed bicone. So $\varpi_{1}(Y)$ and $\varpi_{2}(Y)$ are closed subsets of $\mathfrak{g}$.

We fix a principal $\mathfrak{s l}_{2}$-triple $(e, h, f)$ of $\mathfrak{g}$. Thus, the following relations

$$
[h, e]=2 e, \quad[e, f]=h, \quad[h, f]=-2 f,
$$

are satisfied, $e$ and $f$ are regular nilpotent elements and $h$ is a regular semisimple element of $\mathfrak{g}$. If ad is the adjoint representation of $\mathfrak{g}$, then $\operatorname{ad} h$ induces a $\mathbb{Z}$-grading on $\mathfrak{g}$ and we have $\mathfrak{g}=\sum_{i \in \mathbb{Z}} \mathfrak{g}_{i}$, where $\mathfrak{g}_{i}$ is the $i$-eigenspace of $\operatorname{ad} h$. Moreover, all the eigenvalues of ad $h$ are even integers. The centralizer $\mathfrak{g}(h)=\mathfrak{g}_{0}$ of $h$ in $\mathfrak{g}$ is a Cartan subalgebra, that we will also denote by $\mathfrak{h}$. The unique Borel subalgebra containing $e$ is

$$
\mathfrak{b}:=\mathfrak{h} \oplus \sum_{i>0} \mathfrak{g}_{i}
$$


and the nilpotent radical of $\mathfrak{b}$ is $\mathfrak{u}:=\sum_{i>0} \mathfrak{g}_{i}$. We additionally set

$$
\mathfrak{b}_{-}:=\sum_{i \leq 0} \mathfrak{g}_{i}, \mathfrak{u}_{-}:=\sum_{i<0} \mathfrak{g}_{i}
$$

Let $\mathbf{B}$ and $\mathbf{B}_{-}$be the normalizers of $\mathfrak{b}$ and $\mathfrak{b}_{-}$in $G$ and let $\mathbf{H}$ and $N_{G}(\mathfrak{h})$ be the centralizer and the normalizer of $\mathfrak{h}$ in $G$. Then the quotient $W_{G}(\mathfrak{h})$ of $N_{G}(\mathfrak{h})$ by $\mathbf{H}$ is the Weyl group of $\mathfrak{g}$ with respect to $\mathfrak{h}$.

Let $\mathcal{R}$ be the root system of $(\mathfrak{g}, \mathfrak{h})$, let $\mathcal{R}_{+}$be the positive root system of $\mathcal{R}$ defined by $\mathfrak{b}$, and let $\Pi$ be the basis of $\mathcal{R}_{+}$. For any $\alpha$ in $\mathcal{R}$, we denote by $\mathfrak{g}^{\alpha}$ the corresponding root subspace. Denote by $w_{0}$ the longest element of $W_{G}(\mathfrak{h})$ with respect to $\Pi$. Then $w_{0}(\Pi)$ is equal to $-\Pi$ and for any representative $g_{0}$ of $w_{0}$ in $N_{G}(\mathfrak{h}), g_{0}(\mathfrak{b})$ is equal to $\mathfrak{b}_{-}$.

Let $\langle.,$.$\rangle be a nondegenerate G$-invariant bilinear form on $\mathfrak{g} \times \mathfrak{g}$ which extends the Killing form of the semisimple part of $\mathfrak{g}$. In the remainder of this note, the orthogonality will refer to $\langle.,$.$\rangle .$

Acknowledgment. - We are grateful to Michel Duflo for bringing $\mathbf{M u}$ to our attention. We would like also to thank François Loeser for his e-mails concerning motivic integration questions, and Karin Baur for her comments. In addition, we thank Vladimir Popov for his support and the referees for their numerous and judicious advices and their careful attention to our note.

\section{Characteristic submodule}

In this section, we introduce the characteristic submodule of $\mathfrak{g}$ and we describe some of its properties.

1. Preliminaries. - Let us recall that $p_{1}, \ldots, p_{\mathrm{rkg}}$ are homogeneous generators of $\mathrm{S}(\mathfrak{g})^{\mathfrak{g}}$ of degrees $d_{1}, \ldots, d_{\mathrm{rkg}}$ respectively such that the sequence $d_{1}, \ldots, d_{\mathrm{rkg}}$ is weakly increasing. For $i=1, \ldots, \mathrm{rkg}$, let $\varepsilon_{i}$ be the element of $\mathrm{S}(\mathfrak{g}) \otimes_{\mathbb{C}} \mathfrak{g}$ defined by the following relation:

$$
\left\langle\varepsilon_{i}(x), v\right\rangle=p_{i}^{\prime}(x)(v),
$$

for $x, v$ in $\mathfrak{g}$, where $p_{i}^{\prime}(x)$ is the differential of $p_{i}$ at $x$. The first statement of the following lemma comes from $[\mathbf{R i 3}$ (Lemma 2.1) while the second statement comes from [Kos2] (Theorem 9):

Lemma 4. - Let $x$ be in $\mathfrak{g}$.

i) For $i=1, \ldots, \mathrm{rkg}, \varepsilon_{i}(x)$ belongs to the center of $\mathfrak{g}(x)$. 
ii) The elements $\varepsilon_{1}(x), \ldots, \varepsilon_{\mathrm{rkg}}(x)$ are linearly independent in $\mathfrak{g}$ if and only if $x$ is regular. Moreover, if so, $\varepsilon_{1}(x), \ldots, \varepsilon_{\mathrm{rkg}}(x)$ is a basis of $\mathfrak{g}(x)$.

Let $i$ be in $\{1, \ldots, \mathrm{rkg}\}$. The 2 -order polarizations $p_{i, m, n}$ of $p_{i}$ are defined by the following relation:

$$
p_{i}(a x+b y)=\sum_{m+n=d_{i}} a^{m} b^{n} p_{i, m, n}(x, y),
$$

for any $(a, b)$ in $\mathbb{C}^{2}$ and any $(x, y)$ in $\mathfrak{g} \times \mathfrak{g}$. For $(m, n) \in \mathbb{N}^{2}$ such that $m+$ $n=d_{i}$, let $\varepsilon_{i, m, n}$ be the element of $\mathrm{S}(\mathfrak{g}) \otimes_{\mathbb{C}} \mathrm{S}(\mathfrak{g}) \otimes_{\mathbb{C}} \mathfrak{g}$ such that the linear functional $v \mapsto\left\langle\varepsilon_{i, m, n}(x, y), v\right\rangle$ on $\mathfrak{g}$ is the differential at $x$ of the function $x \mapsto p_{i, m, n}(x, y)$ for $(x, y)$ in $\mathfrak{g} \times \mathfrak{g}$. In particular, $\varepsilon_{i, 0, d_{i}}=0$ for $i=1, \ldots, \mathrm{rkg}$. In addition, since $d_{1}+\cdots+d_{\mathrm{rkg}}$ is equal to $\mathrm{b}_{\mathfrak{g}}$, the cardinality of the family $\left\{\varepsilon_{i, 1, d_{i}-1}, \ldots, \varepsilon_{i, d_{i}, 0}, i=1, \ldots, \mathrm{rkg} \mathfrak{g}\right\}$ is equal to $\mathrm{b}_{\mathfrak{g}}$.

Lemma 5. - For $i=1, \ldots, \operatorname{rkg}$ and $(x, y) \in \mathfrak{g} \times \mathfrak{g}$, we have

$$
\varepsilon_{i}(a x+b y)=\sum_{m=1}^{d_{i}} a^{m-1} b^{d_{i}-m} \varepsilon_{i, m, d_{i}-m}(x, y),
$$

for any $(a, b)$ in $\mathbb{C}^{2}$. In particular, $\varepsilon_{i, 1, d_{i}-1}(x, y)=\varepsilon_{i}(y)$ and $\varepsilon_{i, d_{i}, 0}(x, y)=\varepsilon_{i}(x)$.

Proof. - Let $v$ be in $\mathfrak{g}$. For any $(a, b)$ in $\mathbb{C}^{*} \times \mathbb{C}$, we have

$$
\begin{aligned}
\left\langle\varepsilon_{i}(a x+b y), v\right\rangle & =\left.\frac{\mathrm{d}}{\mathrm{d} t} p_{i}(a x+b y+t v)\right|_{t=0} \\
& =\left.\sum_{m+n=d_{i}} a^{m} b^{n} \frac{\mathrm{d}}{\mathrm{d} t} p_{i, m, n}\left(x+t a^{-1} v, y\right)\right|_{t=0} \\
& =\sum_{m+n=d_{i}} a^{m} b^{n}\left\langle\varepsilon_{i, m, n}(x, y), a^{-1} v\right\rangle,
\end{aligned}
$$

whence the lemma, since $\langle.,$.$\rangle is a nondegenerate G$-invariant bilinear form on $\mathfrak{g} \times \mathfrak{g}$.

Recall that we have introduced the subset

$$
\Omega_{\mathfrak{g}}:=\left\{(x, y) \in \mathfrak{g} \times \mathfrak{g} \mid P_{x, y} \backslash\{0\} \subset \mathfrak{g}_{\text {reg }}, \operatorname{dim} P_{x, y}=2\right\},
$$

which is clearly $G$-invariant. We denote by $\mathfrak{h}^{\prime}, \mathfrak{g}_{2}^{\prime}$ and $\mathfrak{g}_{-2}^{\prime}$ the intersections of $\mathfrak{g}_{\text {reg }}$ with $\mathfrak{h}, \mathfrak{g}_{2}$ and $\mathfrak{g}_{-2}$ respectively. Set:

$$
\mathfrak{u}_{+}:=\sum_{i \geq 4} \mathfrak{g}_{i}
$$

In the following lemma, we explicitly provide elements of $\Omega_{\mathfrak{g}}$. 
Lemma 6. - i) If $x \in \mathfrak{h}^{\prime}+\mathfrak{u}$ and $y \in \mathfrak{g}_{2}^{\prime}+\mathfrak{u}_{+}$, then $(x, y) \in \Omega_{\mathfrak{g}}$.

ii) If $x \in \mathfrak{g}_{-2}^{\prime}$ and $y \in \mathfrak{g}_{2}^{\prime}$, then $(x, y) \in \Omega_{\mathfrak{g}}$.

Proof. - i) The elements $x$ and $y$ are clearly linearly independent. In addition, for any $t$ in $\mathbb{C}, x+t y$ is conjugate to $x$ under $G$. So $x+t y$ is regular for any $t$ in $\mathbb{C}$. Since $y$ is regular, $(x, y)$ belongs to $\Omega_{\mathfrak{g}}$.

ii) The elements $x$ and $y$ are clearly linearly independent. As $x$ is regular, $x+s y$ is regular for any $s$ in an open subset of $\mathbb{C}$ containing 0 . Let $t \mapsto g(t)$ be the one-parameter subgroup of $\mathbf{H}$ generated by ad $h$. As $\mathfrak{g}_{\mathrm{reg}}$ is $G$-invariant, for any $t$ in $\mathbb{C}^{*}, g(t)(x+s y)$ is regular for any $s$ in an open subset of $\mathbb{C}$ containing 0 . So, from the relations $[h, x]=-2 x$ and $[h, y]=2 y$, we deduce that for any $t$ in $\mathbb{C}^{*}, t^{-2} x+s t^{2} y$ is regular for any $s$ in an open subset of $\mathbb{C}$ containing 0 . As $\mathfrak{g}_{\text {reg }}$ is an open cone, $x+s t^{4} y$ is consequently regular for any $s$ in an open subset of $\mathbb{C}$ containing $\{0\}$. So $x+t y$ is regular for any $t$ in $\mathbb{C}$. As $y$ is regular, we deduce that $(x, y)$ belongs to $\Omega_{\mathfrak{g}}$.

Remark 1. - The subset $\Omega_{\mathfrak{g}}$ is stable under the involution $(x, y) \mapsto(y, x)$. So, by Lemma 6, the images of the subsets $\left(\mathfrak{h}^{\prime}+\mathfrak{u}\right) \times\left(\mathfrak{g}_{2}^{\prime}+\mathfrak{u}_{+}\right)$and $\mathfrak{g}_{-2}^{\prime} \times \mathfrak{g}_{2}^{\prime}$ by this involution are contained in $\Omega_{\mathfrak{g}}$. For example, the elements $(e, h),(f, h)$ and $(e, f)$ are in $\Omega_{\mathfrak{g}}$.

The following lemma is well-known:

Lemma 7. - Let $x$ be in $\mathfrak{g}_{\mathrm{reg}}$.

i) If $x$ belongs to $\mathfrak{b}$, then $\mathfrak{g}(x)$ is contained in $\mathfrak{b}$.

ii) If $x$ belongs to $\mathfrak{u}$, then $\mathfrak{g}(x)$ is contained in $\mathfrak{u}$.

For $(x, y)$ in $\mathfrak{g} \times \mathfrak{g}$, we denote by $\mathfrak{V}(x, y)$ the subspace generated by the elements

and we set:

$$
\varepsilon_{i, 1, d_{i}-1}(x, y), \ldots, \varepsilon_{i, d_{i}, 0}(x, y), i=1, \ldots, \mathrm{rkg},
$$

$$
\mathfrak{V}^{\prime}(x, y):=\sum_{(a, b) \in \mathbb{C}^{2} \backslash(0,0)} \mathfrak{g}(a x+b y) .
$$

We collect in the following lemma some results concerning the subset $\Omega_{\mathfrak{g}}$ in part obtained by A. V. Bolsinov in Bol and recently by D. I. Panyushev and O. Yakimova in $[\mathbf{P Y a}$ :

Lemma 8. - Let $(x, y)$ be in $\mathfrak{g} \times \mathfrak{g}$.

i) The subspace $\mathfrak{V}(x, y)$ has dimension at most $\mathrm{b}_{\mathfrak{g}}$. Moreover, it has dimension $\mathrm{b}_{\mathfrak{g}}$ if and only if $(x, y)$ belongs to $\Omega_{\mathfrak{g}}$.

ii) The subspace $\mathfrak{V}(x, y)$ is contained in $\mathfrak{V}^{\prime}(x, y)$. Moreover, the equality occurs when $(x, y)$ belongs to $\Omega_{\mathfrak{g}}$.

iii) If $(x, y)$ belongs to $\mathfrak{b} \times \mathfrak{b}$, then $\mathfrak{V}(x, y)$ is contained in $\mathfrak{b}$. 
Proof. - i) As the sum of $d_{1}, \ldots, d_{\mathrm{rkg}}$ is equal to $\mathrm{b}_{\mathfrak{g}}, \mathfrak{V}(x, y)$ has dimension at most $b_{\mathfrak{g}}$. Let us suppose that $(x, y)$ belongs to $\Omega_{\mathfrak{g}}$. Then by $\mathbf{P Y a}$ (Theorem 2.4 ), the subspace $\mathfrak{V}(x, y)$ has dimension $b_{\mathfrak{g}}$. Conversely, let us suppose that $\mathfrak{V}(x, y)$ has dimension $b_{\mathfrak{g}}$. In particular, $\varepsilon_{1}(x), \ldots, \varepsilon_{\mathrm{rkg}}(x)$ are linearly independent. Hence by Lemma 4, (ii), $x$ is regular. By Lemma 5, for any $(a, b)$ in $\mathbb{C}^{2} \backslash\{(0,0)\}, \mathfrak{V}(x, y)$ is equal to $\mathfrak{V}(a x+b y, y)$. Hence $(x, y)$ belongs to $\Omega_{\mathfrak{g}}$.

ii) By Lemma 4, $\mathfrak{V}^{\prime}(x, y)$ contains $\varepsilon_{i}(a x+b y)$ for $i=1, \ldots$, rkg and any $(a, b)$ in $\mathbb{C}^{2}$. Hence by Lemma 5 , $\mathfrak{V}^{\prime}(x, y)$ contains $\mathfrak{V}(x, y)$. Moreover, by (i) and Lemma 4, $\mathfrak{V}(x, y)$ is equal to $\mathfrak{V}^{\prime}(x, y)$ when $(x, y)$ belongs to $\Omega_{\mathfrak{g}}$.

iii) Let us suppose that $(x, y)$ belongs to $\mathfrak{b} \times \mathfrak{b}$. By Lemma 6 and Remark 1 . the intersection of $\Omega_{\mathfrak{g}}$ and $\mathfrak{b} \times \mathfrak{b}$ is a nonempty open subset. Moreover, when $(x, y)$ belongs to this intersection, for any $(a, b)$ in $\mathbb{C}^{2} \backslash\{(0,0)\}, \mathfrak{g}(a x+b y)$ is contained in $\mathfrak{b}$, by Lemmas 4 , (ii) and 7 , (i). Hence for $i=1, \ldots$, rk $\mathfrak{g}$ and $m=1, \ldots, d_{i}, \varepsilon_{i, m, d_{i}-m}(x, y)$ belongs to $\mathfrak{b}$, by (ii). So $\mathfrak{V}(x, y)$ is contained in $\mathfrak{b}$.

2. Closed irreducible subsets of $\mathfrak{s} \times \mathfrak{s}$ invariant under the actions of $\mathbf{S}$ and $\mathrm{GL}_{2}(\mathbb{C})$. - The following automorphisms give an action of $\mathrm{GL}_{2}(\mathbb{C})$ in $\mathfrak{g} \times \mathfrak{g}$ :

$$
(x, y) \longmapsto(a x+b y, c x+d y), \text { where } g=\left[\begin{array}{ll}
a & b \\
c & d
\end{array}\right] .
$$

Let $\mathfrak{s}$ be the subspace of $\mathfrak{g}$ generated by $e, h, f$ so that $\mathfrak{s}$ is isomorphic to $\mathfrak{s l}_{2}(\mathbb{C})$. Let $\mathbf{S}$ be the closed connected subgroup of $G$ whose Lie algebra is ad $\mathfrak{s}$. We start this subsection by describing the closed irreducible subsets of $\mathfrak{s} \times \mathfrak{s}$ invariant under the actions of $\mathbf{S}$ and $\mathrm{GL}_{2}(\mathbb{C})$. This will be used in Corollary 10 and Lemma 18 .

Let $T_{3}$ be the closed bicone of $\mathfrak{s} \times \mathfrak{s}$ generated by the diagonal of $\mathfrak{N}_{\mathfrak{s}} \times \mathfrak{N}_{\mathfrak{s}}$, let $T_{4}$ be the closed bicone of $\mathfrak{s} \times \mathfrak{s}$ generated by the diagonal of $\mathfrak{s} \times \mathfrak{s}$ and let $T_{5}$ be the subset of elements $(x, y)$ of $\mathfrak{s} \times \mathfrak{s}$ such that $x$ and $y$ belong to the same Borel subalgebra of $\mathfrak{s}$. As defined, the sets $T_{3}, T_{4}$ and $T_{5}$ are irreducible closed subsets of $\mathfrak{s} \times \mathfrak{s}$, invariant under the actions of $\mathbf{S}$ and $\mathrm{GL}_{2}(\mathbb{C})$. Moreover, they have dimension 3,4 and 5 respectively. The verification of these claims is left to the reader.

Lemma 9. - The subsets $\{0\}, T_{3}, T_{4}, T_{5}$ and $\mathfrak{s} \times \mathfrak{s}$ are the only nonempty irreducible closed subsets of $\mathfrak{s} \times \mathfrak{s}$, invariant under the actions of $\mathbf{S}$ and $\mathrm{GL}_{2}(\mathbb{C})$.

Proof. - Let us first remark that $\mathfrak{N}_{\mathfrak{s}}$ is the only proper $\mathbf{S}$-invariant closed cone of $\mathfrak{s}$. Let $T$ be a nonempty irreducible closed subset of $\mathfrak{s} \times \mathfrak{s}$, invariant under the actions of $\mathbf{S}$ and $\mathrm{GL}_{2}(\mathbb{C})$. By Lemma 3 and the preceding remark, if $0<\operatorname{dim} T<4$, then $\varpi_{1}(T)$ is equal to $\mathfrak{N}_{\mathfrak{s}}$, whence $T=T_{3}$ by $\mathrm{GL}_{2}(\mathbb{C})$ invariance. We assume now $\operatorname{dim} T \geq 4$. As $\varpi_{1}(T) \supset \mathfrak{N}_{\mathfrak{s}}$ and $\operatorname{dim} T \geq 4, T$ 
contains an element $(e, a e+b f+c h)$, with $b$ or $c$ different from 0 . Hence $T$ contains $(e, b f+c h)$. If $c \neq 0$, then $\varpi_{1}(T)$ contains $b f+c h$ which is semisimple. Otherwise, $T$ contains $(e, f)$ and $\varpi_{1}(T)$ contains $e+f$ which is semisimple, too. So, in any case, $\varpi_{1}(T)=\mathfrak{s}$. In particular, $T \supset T_{4}$ and the equality holds as soon $\operatorname{as} \operatorname{dim} T=4$. At last, let us suppose $\operatorname{dim} T \geq 5$. As $\varpi_{1}(T)=\mathfrak{s}$, there exist $a, b, c$ in $\mathbb{C}$ such that $(h, a e+b f+c h)$ belongs to $T$ with $a$ or $b$ different from 0 . If $a b=0$, using the invariance of $T$ under $\mathbf{S}$ and $\mathrm{GL}_{2}(\mathbb{C})$, we deduce that $\{h\} \times \mathfrak{b}_{\mathfrak{s}}$ is contained in $T$. In this case, $T$ contains $T_{5}$. If $a b \neq 0$, using the invariance of $T$ under $\mathrm{GL}_{2}(\mathbb{C})$ and the one-parameter subgroup of $\mathbf{H}$ generated by ad $h$, we deduce that $\left(h, a t^{2} e+b t^{-2} f\right)$ belongs to $T$ for any $t$ in $\mathbb{C}^{*}$. So $\{h\} \times \mathfrak{s}$ is contained in $T$ and $T$ has dimension 6 , whence the lemma.

Corollary 10. - The subset $\Omega_{\mathfrak{g}}$ is a big open subset of $\mathfrak{g} \times \mathfrak{g}$.

Proof. - Suppose that $\Omega_{\mathfrak{g}}$ is not a big open subset of $\mathfrak{g} \times \mathfrak{g}$. Then, the complementary of $\Omega_{\mathfrak{g}}$ in $\mathfrak{g} \times \mathfrak{g}$ has an irreducible component $\Sigma$ of codimension 1 in $\mathfrak{g} \times \mathfrak{g}$. As $\Omega_{\mathfrak{g}}$ is invariant under the action of $G$ and $\mathrm{GL}_{2}(\mathbb{C}), \Sigma$ is invariant under these actions too. The intersection $T$ of $\Sigma$ and $\mathfrak{s} \times \mathfrak{s}$ contains $(0,0)$. So $T$ is a nonempty closed cone of $\mathfrak{s} \times \mathfrak{s}$, invariant under the actions of $\mathbf{S}$ and $\mathrm{GL}_{2}(\mathbb{C})$. As $\Sigma$ is an hypersurface of $\mathfrak{g} \times \mathfrak{g}, T$ has codimension at most 1 in $\mathfrak{s} \times \mathfrak{s}$. Hence Lemma 9, (ii), implies that $(h, e)$ belongs to $T$. But by Lemma 6. (i), $(h, e)$ belongs to $\Omega_{\mathfrak{g}}$, whence the expected contradiction.

Remark 2. - We can also deduce Corollary 10 from [PYa. Indeed, according to $\left[\mathbf{P Y a}\right.$ (Lemma 3.1), for any $x \in \mathfrak{g}_{\text {reg }}$, the set $\left\{y \in \mathfrak{g} \mid(x, y) \in \Omega_{\mathfrak{g}}\right\}$ is a big open subset of $\mathfrak{g}$, whence we can readily deduce Corollary 10 .

3. Characteristic submodule. - By a result of J. Dixmier Di ( $(\S 2)$, the $\mathrm{S}(\mathfrak{g})$-submodule of elements $\varphi$ in $\mathrm{S}(\mathfrak{g}) \otimes_{\mathbb{C}} \mathfrak{g}$ such that $\varphi(x)$ belongs to $\mathfrak{g}(x)$ for all $x$ in $\mathfrak{g}$ is a free module of basis $\varepsilon_{1}, \ldots, \varepsilon_{\mathrm{rkg}}$.

Definition 1. - The characteristic submodule $\mathrm{B}_{\mathfrak{g}}$ of $\mathfrak{g}$ is the $\mathrm{S}(\mathfrak{g}) \otimes_{\mathbb{C}} \mathrm{S}(\mathfrak{g})$ submodule of elements $\varphi$ in $\mathrm{S}(\mathfrak{g}) \otimes_{\mathbb{C}} \mathrm{S}(\mathfrak{g}) \otimes_{\mathbb{C}} \mathfrak{g}$ such that $\varphi(x, y)$ belongs to $\mathfrak{V}^{\prime}(x, y)$, for any $(x, y)$ in a nonempty open subset of $\mathfrak{g} \times \mathfrak{g}$.

The following result can be viewed as a generalization of the previous result of J. Dixmier.

Theorem 11. - The submodule $\mathrm{B}_{\mathfrak{g}}$ of $\mathrm{S}(\mathfrak{g}) \otimes_{\mathbb{C}} \mathrm{S}(\mathfrak{g}) \otimes_{\mathbb{C}} \mathfrak{g}$ is a free $\mathrm{S}(\mathfrak{g}) \otimes_{\mathbb{C}} \mathrm{S}(\mathfrak{g})$-module of rank $\mathrm{b}_{\mathfrak{g}}$. Moreover, the family $\left\{\varepsilon_{i, 1, d_{i}-1}, \ldots, \varepsilon_{i, d_{i}, 0}\right.$, $i=1, \ldots, \mathrm{rk} \mathfrak{g}\}$ is a basis of $\mathrm{B}_{\mathfrak{g}}$. 
Proof. - By Lemma 8, (ii), for $i=1, \ldots$, rkg and $m=1, \ldots, d_{i}, \varepsilon_{i, m, d_{i}-m}$ belongs to $\mathrm{B}_{\mathfrak{g}}$. Moreover, by Lemma 8 , (i), these elements are linearly independent over $S(\mathfrak{g}) \otimes_{\mathbb{C}} S(\mathfrak{g})$. It remains to prove that they generate $B_{\mathfrak{g}}$ as $\mathrm{S}(\mathfrak{g}) \otimes_{\mathbb{C}} \mathrm{S}(\mathfrak{g})$-module. Let $\varphi$ be in $\mathrm{B}_{\mathfrak{g}}$. By Lemma 8 , (i) and (ii), $\varphi(x, y)$ belongs to $\mathfrak{V}(x, y)$ for any $(x, y)$ in $\Omega_{\mathfrak{g}}$. So there exist regular functions $\psi_{i, m, d_{i}-m}$ on $\Omega_{\mathfrak{g}}$ for $i=1, \ldots, \mathrm{rkg}$ and $m=1, \ldots, d_{i}$, such that:

$$
\varphi(x, y)=\sum_{\substack{i=1, \ldots, \mathrm{rkg} \\ m=1, \ldots, d_{i}}} \psi_{i, m, d_{i}-m}(x, y) \varepsilon_{i, m, d_{i}-m}(x, y),
$$

for any $(x, y)$ in $\Omega_{\mathfrak{g}}$. By Corollary 10, $\Omega_{\mathfrak{g}}$ is a big open subset of $\mathfrak{g} \times \mathfrak{g}$. Hence the regular functions $\psi_{i, m, d_{i}-m}$ have regular extensions to $\mathfrak{g} \times \mathfrak{g}$ since $\mathfrak{g} \times \mathfrak{g}$ is normal. As a result, the family $\left\{\varepsilon_{i, 1, d_{i}-1}, \ldots, \varepsilon_{i, d_{i}, 0}, i=1, \ldots, \mathrm{rk} \mathfrak{g}\right\}$ is a basis of $\mathrm{B}_{\mathfrak{g}}$.

4. Smooth points of $\mathcal{N}_{\mathfrak{g}}$. - In this subsection, we establish a link between the open subset $\Omega_{\mathfrak{g}}$ and the nilpotent bicone. For $i=1, \ldots$, rk $\mathfrak{g}$, we denote by $\sigma_{i}$ the map

$$
\mathfrak{g} \times \mathfrak{g} \longrightarrow \mathbb{C}^{d_{i}+1},(x, y) \mapsto\left(p_{i, 0, d_{i}}(x, y), \ldots, p_{i, d_{i}, 0}(x, y)\right) .
$$

Lemma 12. - For $(x, y)$ in $\mathfrak{g} \times \mathfrak{g}$ and $i=1, \ldots, \mathrm{rk} \mathfrak{g}$, the differential of $\sigma_{i}$ at $(x, y)$ is the linear map

$$
\begin{gathered}
(v, w) \longmapsto\left(\left\langle\varepsilon_{i, 1, d_{i}-1}(x, y), w\right\rangle,\left\langle\varepsilon_{i, 1, d_{i}-1}(x, y), v\right\rangle+\left\langle\varepsilon_{i, 2, d_{i}-2}(x, y), w\right\rangle,\right. \\
\left.\quad \cdots,\left\langle\varepsilon_{i, d_{i}-1,1}(x, y), v\right\rangle+\left\langle\varepsilon_{i, d_{i}, 0}(x), w\right\rangle,\left\langle\varepsilon_{i}(x), v\right\rangle\right) .
\end{gathered}
$$

Proof. - For $(x, y)$ in $\mathfrak{g} \times \mathfrak{g}$, we denote by $p_{i, m, d_{i}-m}^{\prime}(x, y)$ the differential of $p_{i, m, d_{i}-m}$ at $(x, y)$. From Lemma 5 and the equality

$$
p_{i}(t x+y)=\sum_{m=0}^{d_{i}} t^{m} p_{i, m, d_{i}-m}(x, y)
$$

for $(x, y)$ in $\mathfrak{g} \times \mathfrak{g}$, we deduce the equality

$$
\sum_{m=1}^{d_{i}} t^{m}\left\langle\varepsilon_{i, m, d_{i}-m}(x, y), v+t^{-1} w\right\rangle=\sum_{m=0}^{d_{i}} t^{m} p_{i, m, d_{i}-m}^{\prime}(x, y)(v, w),
$$

for $x, y, v, w$ in $\mathfrak{g}$ and $t$ in $\mathbb{C}^{*}$, since $p_{i, 0, d_{i}}$ is the map $(x, y) \mapsto p_{i}(y)$. Hence we get, for $m=0, \ldots, d_{i}-1$,

$$
p_{i, m, d_{i}-m}^{\prime}(x, y)(v, w)=\left\langle\varepsilon_{i, m, d_{i}-m}(x, y), v\right\rangle+\left\langle\varepsilon_{i, m+1, d_{i}-m-1}(x, y), w\right\rangle .
$$

In addition $p_{i, d_{i}, 0}^{\prime}(x, y)$ is the linear functional $(v, w) \mapsto\left\langle\varepsilon_{i}(x), v\right\rangle$. 
Let $\sigma$ be the map:

$$
\mathfrak{g} \times \mathfrak{g} \longrightarrow \mathbb{C}^{\mathrm{b}_{\mathfrak{g}}+\mathrm{rkg}},(x, y) \mapsto\left(\sigma_{1}(x, y), \ldots, \sigma_{\mathrm{rkg}}(x, y)\right) .
$$

Proposition 13. - Let $(x, y)$ be in $\mathfrak{g} \times \mathfrak{g}$. Then $\sigma$ is smooth at $(x, y)$ if and only if $(x, y)$ belongs to $\Omega_{\mathfrak{g}}$.

Proof. - We denote by $\sigma^{\prime}(x, y)$ the differential of $\sigma$ at $(x, y)$ and we denote by $\operatorname{ker} \sigma^{\prime}(x, y)$ its kernel in $\mathfrak{g} \times \mathfrak{g}$. Let us suppose that $(x, y)$ belongs to $\Omega_{\mathfrak{g}}$. For $v$ in $\mathfrak{g}$, we denote by $X_{v}$ the subset of elements $w$ in $\mathfrak{g}$ such that $(v, w)$ is in $\operatorname{ker} \sigma^{\prime}(x, y)$. By Lemmas 12 and 4 , (ii), $v$ belongs to the orthogonal complement of $\mathfrak{g}(x)$ in $\mathfrak{g}$. In addition, $X_{v}$ is an affine subspace whose tangent space is equal to the orthogonal complement of $\mathfrak{V}(x, y)$ in $\mathfrak{g}$. By Lemma 8 , (i), $\mathfrak{V}(x, y)$ has dimension $\mathrm{b}_{\mathfrak{g}}$ since $(x, y)$ belongs to $\Omega_{\mathfrak{g}}$. Consequently, $\operatorname{ker} \sigma^{\prime}(x, y)$ has dimension at most $\mathrm{b}_{\mathfrak{g}}-\mathrm{rk} \mathfrak{g}+2\left(\mathrm{~b}_{\mathfrak{g}}-\mathrm{rk} \mathfrak{g}\right)=3\left(\mathrm{~b}_{\mathfrak{g}}-\mathrm{rk} \mathfrak{g}\right)$, since $x$ is regular. Hence the image of $\sigma^{\prime}(x, y)$ has dimension at least $b_{\mathfrak{g}}+\mathrm{rkg}$. So $\sigma^{\prime}(x, y)$ is surjective and $\sigma$ is smooth at $(x, y)$.

Conversely, suppose that $\sigma$ is smooth at $(x, y)$. For $(a, b)$ in $\mathbb{C}^{2}$, we denote by $\pi_{a, b}$ the linear map

$$
\left(z_{i, 0, d_{i}}, \ldots, z_{i, d_{i}, 0}, i=1, \ldots, \mathrm{rk} \mathfrak{g}\right) \longmapsto\left(\sum_{m+n=d_{i}} a^{m} b^{n} z_{i, m, n}, i=1, \ldots, \mathrm{rk} \mathfrak{g}\right)
$$

from $\mathbb{C}^{\mathrm{b}_{\mathfrak{g}}+\mathrm{rkg}}$ to $\mathbb{C}^{\mathrm{rkg}}$. The linear map $\pi_{a, b}$ is surjective as soon as $(a, b) \neq(0,0)$. Since $\sigma$ is smooth at $(x, y)$, we deduce that the compound map $\sigma_{a, b}:=\pi_{a, b^{\circ} \sigma}$ is smooth at $(x, y)$, for any $(a, b)$ in $\mathbb{C}^{2} \backslash\{(0,0)\}$. As

$$
\sum_{m+n=d_{i}} a^{m} b^{n} p_{i, m, n}\left(x^{\prime}, y^{\prime}\right)=p_{i}\left(a x^{\prime}+b y^{\prime}\right),
$$

for $i=1, \ldots, \mathrm{rkg}$ and $\left(x^{\prime}, y^{\prime}\right)$ in $\mathfrak{g} \times \mathfrak{g}, \sigma_{a, b}$ maps $\left(x^{\prime}, y^{\prime}\right)$ to $\left(p_{1}\left(a x^{\prime}+b y^{\prime}\right), \ldots, p_{\mathrm{rkg}}\left(a x^{\prime}+b y^{\prime}\right)\right)$. Moreover, it is the compound map of the two following maps:

$$
\begin{array}{r}
\mathfrak{g} \times \mathfrak{g} \longrightarrow \mathfrak{g},\left(x^{\prime}, y^{\prime}\right) \mapsto\left(a x^{\prime}+b y^{\prime}\right) \\
\mathfrak{g} \longrightarrow \mathbb{C}^{\mathrm{rkg}}, z \mapsto\left(p_{1}(z), \ldots, p_{\mathrm{rkg}}(z)\right) .
\end{array}
$$

Therefore, the second map is smooth at $(a x+b y)$, for any $(a, b)$ in $\mathbb{C}^{2} \backslash\{(0,0)\}$. So, by Lemma 4, (ii), $(x, y)$ belongs to $\Omega_{\mathfrak{g}}$.

Remark 3. - Recall that $\mathcal{N}_{\mathfrak{g}}$ is the subscheme of $\mathfrak{g} \times \mathfrak{g}$ defined by the ideal generated by the polynomials $p_{i, m, n}$. Therefore, by Proposition 13, the intersection of $\mathcal{N}_{\mathfrak{g}}$ and $\Omega_{\mathfrak{g}}$ is nothing but the set of smooth points of $\mathcal{N}_{\mathfrak{g}}$. The elements of $\Omega_{\mathfrak{g}}$ provided by Lemma 6, (i), do not belong to $\mathcal{N}_{\mathfrak{g}}$. Consider the 
elements described in Lemma 6. (ii). If $\mathfrak{g}$ is equal to $\mathfrak{s l}_{3}$, the subset $\mathfrak{g}_{-2}^{\prime} \times \mathfrak{g}_{2}^{\prime}$ has a nonempty intersection with $\mathcal{N}_{\mathfrak{g}}$. Indeed, if we set

$$
e:=\left[\begin{array}{lll}
0 & 1 & 0 \\
0 & 0 & 1 \\
0 & 0 & 0
\end{array}\right], h:=\left[\begin{array}{ccc}
2 & 0 & 0 \\
0 & 0 & 0 \\
0 & 0 & -2
\end{array}\right], x:=\left[\begin{array}{ccc}
0 & 0 & 0 \\
1 & 0 & 0 \\
0 & -1 & 0
\end{array}\right],
$$

then $(x, e) \in \mathcal{N}_{\mathfrak{g}} \cap\left(\mathfrak{g}_{-2}^{\prime} \times \mathfrak{g}_{2}^{\prime}\right)$. But in general this intersection can be empty. For instance it is an empty set if $\mathfrak{g}=\mathfrak{s l}_{n}$ for many $n>3$. Whatever the case, we will see a posteriori that there exists at least one irreducible component of $\mathcal{N}_{\mathfrak{g}}$ which has an empty intersection with $\Omega_{\mathfrak{g}}$ (see Theorem 45). So we cannot hope to apply the criterion of Kostant [Kos2]. This observation was originally the reason why we introduced an other subscheme (see the following section).

\section{Principal cone and principal bicone}

In this section, we suppose that $\mathfrak{g}$ is simple. Then we can suppose that $p_{1}$ is the Casimir element of $\mathrm{S}(\mathfrak{g})^{\mathfrak{g}}$, that is $p_{1}(x)=\langle x, x\rangle$, for any $x$ in $\mathfrak{g}$. Recall that $(e, h, f)$ is a principal $\mathfrak{s l}_{2}$-triple of $\mathfrak{g}$ and use the notations of the Introduction, \$3.

1. Principal cone. - Since $e$ is a regular nilpotent element of $\mathfrak{g}$, the nilpotent cone $\mathfrak{N}_{\mathfrak{g}}$ is the $G$-invariant closed cone generated by $e$. According to Kostant's results Kos2, the nilpotent cone is a complete intersection of codimension rkg. Moreover, it is proved in $[\mathbf{H e}]$ that it has rational singularities. In this subsection, we intend to prove analogous properties for the principal cone introduced in:

Definition 2. - The principal cone $\mathfrak{X}_{\mathfrak{g}}$ of $\mathfrak{g}$ is the $G$-invariant closed cone generated by $h$.

Recall that $w_{0}$ is the longest element of the Weyl group $W_{G}(\mathfrak{h})$. The simple following well-known lemma turns out to be useful.

Lemma 14. - The element $w_{0}(h)$ is equal to $-h$. Moreover, there exists a representative $g_{0}$ of $w_{0}$ in $N_{G}(\mathfrak{h})$ such that $g_{0}(e)$ is equal to $f$.

For $i=2, \ldots, \mathrm{rk} \mathfrak{g}$, we define the element $q_{i}$ of $\mathrm{S}(\mathfrak{g})^{\mathfrak{g}}$ as follows:

$$
q_{i}= \begin{cases}p_{i}, & \text { if } d_{i} \text { is odd } \\ p_{1}(h)^{d_{i} / 2} p_{i}-p_{i}(h) p_{1}^{d_{i} / 2}, & \text { otherwise }\end{cases}
$$

The polynomial $q_{i}$ is homogeneous of degree $d_{i}$. As the eigenvalues of ad $h$ are integers, not all equal to zero, $p_{1}(h) \neq 0$. In addition, as

$$
p_{i}(h)=p_{i}\left(w_{0}(h)\right)=p_{i}(-h)=(-1)^{d_{i}} p_{i}(h),
$$


$p_{i}(h)=0$ as soon as $d_{i}$ is odd. This forces $q_{i}(h)=0$, for any $i=2, \ldots$, rk $\mathfrak{g}$. So $\mathfrak{X}_{\mathfrak{g}}$ is contained in the nullvariety of the functions $q_{2}, \ldots, q_{\mathrm{rkg}}$.

Lemma 15. - i) The nullvariety of $p_{1}$ in $\mathfrak{X}_{\mathfrak{g}}$ is equal to $\mathfrak{N}_{\mathfrak{g}}$.

ii) The principal cone $\mathfrak{X}_{\mathfrak{g}}$ is the nullvariety of the functions $q_{2}, \ldots, q_{\mathrm{rkg}}$.

iii) The subset $\left(\mathfrak{X}_{\mathfrak{g}} \backslash \mathfrak{g}_{\text {reg }}\right)$ is equal to $\left(\mathfrak{N}_{\mathfrak{g}} \backslash\right.$ G.e) and the codimension of $\left(\mathfrak{X}_{\mathfrak{g}} \backslash \mathfrak{g}_{\text {reg }}\right)$ in $\mathfrak{X}_{\mathfrak{g}}$ is equal to 3 .

Proof. - i) Prove first that $\mathfrak{N}_{\mathfrak{g}}$ is contained in $\mathfrak{X}_{\mathfrak{g}}$. Since $\mathfrak{X}_{\mathfrak{g}}$ is a $G$-invariant closed cone, the relation $\exp (-\operatorname{tad} e)(h)=h+2$ te for any $t \in \mathbb{C}$, implies $e \in \mathfrak{X}_{\mathfrak{g}}$. So $\mathfrak{N}_{\mathfrak{g}}$ is contained in $\mathfrak{X}_{\mathfrak{g}}$ as closure of G.e. Let $X$ be the nullvariety of the functions $q_{2}, \ldots, q_{\mathrm{rkg}}$. As $p_{1}(h) \neq 0$, the nullvariety of $p_{1}$ in $X$ is the nullvariety of $p_{1}, \ldots, p_{\mathrm{rkg}}$. So $\mathfrak{N}_{\mathfrak{g}}$ is the nullvariety of $p_{1}$ in $X$. Moreover, $\mathfrak{N}_{\mathfrak{g}}$ is also the nullvariety of $p_{1}$ in $\mathfrak{X}_{\mathfrak{g}}$ since $\mathfrak{X}_{\mathfrak{g}}$ is a subset of $X$ which contains $\mathfrak{N}_{\mathfrak{g}}$.

ii) Let $X$ be as in (i). We need to prove that $X$ is contained in $\mathfrak{X}_{\mathfrak{g}}$. Let $x$ be an element of $X$ which is not nilpotent. By (i), $p_{1}(x) \neq 0$. So there exists $t \in \mathbb{C}^{*}$ such that $p_{1}(t x)=p_{1}(h)$. Then $p_{i}(t x)=p_{i}(h)$, for $i=1, \ldots$, rk $\mathfrak{g}$ since $x$ is in $X$. As $h$ is regular and semisimple, $t x$ and $h$ are $G$-conjugate. Hence $x$ belongs to $\mathfrak{X}_{\mathfrak{g}}$.

iii) By (i) and (ii), the subset of elements of $\mathfrak{X}_{\mathfrak{g}}$ which are not regular in $\mathfrak{g}$ is equal to $\left(\mathfrak{N}_{\mathfrak{g}} \backslash\right.$ G.e). As this subset has codimension 2 in $\mathfrak{N}_{\mathfrak{g}}$, it has codimension 3 in $\mathfrak{X}_{\mathfrak{g}}$ by (i).

Recall that $\mathfrak{b}_{-}$is the Borel subalgebra containing $\mathfrak{h}$ and "opposite" to $\mathfrak{b}$, with nilpotent radical $\mathfrak{u}_{-}$. Let $\mathfrak{b}_{0}$ be the subspace of $\mathfrak{b}$ generated by $h$ and $\mathfrak{u}$ and let $\mathfrak{b}_{0,-}$ be the subspace of $\mathfrak{b}_{-}$generated by $h$ and $\mathfrak{u}_{-}$. The following results are partially proved in $[\mathbf{R i 2}$ (Proposition 10.3) as pointed out in Remark 4 below.

Corollary 16. - The principal cone of $\mathfrak{g}$ is normal and it is a complete intersection of codimension $\mathrm{rk} \mathfrak{g}-1$ in $\mathfrak{g}$. The regular elements of $\mathfrak{g}$ which belong to $\mathfrak{X}_{\mathfrak{g}}$ are smooth points of $\mathfrak{X}_{\mathfrak{g}}$. At last, $\mathfrak{X}_{\mathfrak{g}}$ has rational singularities.

Proof. - By Lemma 15, (i) and (ii), $\mathfrak{X}_{\mathfrak{g}}$ is a complete intersection of codimension rkg -1 . By Lemma 4, (ii), the differentials at $x$ of $p_{1}, \ldots, p_{\text {rkg }}$ are linearly independent as soon as $x$ is a regular element of $\mathfrak{g}$. Hence the same goes for the differentials at $x$ of $q_{2}, \ldots, q_{\mathrm{rkg}}$. Then, any $x$ in the union of G.e and $\left(\mathbb{C}^{*}\right) G . h$ is a smooth point of $\mathfrak{X}_{\mathfrak{g}}$. So by Lemma 15, (ii), $\mathfrak{X}_{\mathfrak{g}}$ is regular in codimension 1. By Serre's normality criterion $[\mathbf{M a}]\left(\right.$ Ch. 8, Theorem 23.8), $\mathfrak{X}_{\mathfrak{g}}$ is a normal subvariety.

The subalgebra $\mathfrak{b}_{0}$ is an ideal of $\mathfrak{b}$. The contracted product $G \times_{\mathbf{B}} \mathfrak{b}_{0}$ is defined as the quotient of $G \times \mathfrak{b}_{0}$ under the right action of $\mathbf{B}$ given by $(g, x) . b=$ $\left(g b, b^{-1}(x)\right)$. The map $(g, x) \mapsto g(x)$ from $G \times \mathfrak{b}_{0}$ to $\mathfrak{g}$ factorizes through the canonical map from $G \times \mathfrak{b}_{0}$ to $G \times \times_{\mathbf{B}} \mathfrak{b}_{0}$. Since $G \cdot \mathfrak{b}_{0}$ is equal to $\mathfrak{X}_{\mathfrak{g}}$, we get 
a surjective morphism $\pi$ from $G \times_{\mathbf{B}} \mathfrak{b}_{0}$ to $\mathfrak{X}_{\mathfrak{g}}$. Moreover, the morphism $\pi$ is proper since $G / \mathbf{B}$ is a projective variety. Let $K$ be the field of rational functions on $G \times_{\mathbf{B}} \mathfrak{b}_{0}$ and let $K_{0}$ be the field of rational functions on $\mathfrak{X}_{\mathfrak{g}}$. Let $g_{0}$ be as in Lemma 14. By Lemma 14, $g_{0}(h)$ is equal to $-h$. In particular, the fiber of $\pi$ at $h$ has at least two elements. Let $(g, x)$ be an element of $G \times \mathfrak{b}_{0}$ such that $g(x)$ is equal to $h$. In particular, $x$ is a regular semisimple element of $\mathfrak{g}$ which belongs to $\mathfrak{b}_{0}$ Hence there exists $b$ in $\mathbf{B}$ such that $b(x)$ belongs to the line generated by $h$. As ad $h$ and $\operatorname{ad} b(x)$ have the same eigenvalues, $b(x)$ is equal to $h$ or $-h$, since the eigenvalues of ad $h$ are integers, not all equal to zero. If $b(x)=h$, then $(g, x)$ and $\left(\mathbf{1}_{\mathfrak{g}}, h\right)$ are equal in $G \times{ }_{\mathbf{B}} \mathfrak{b}_{0}$. Otherwise, $(g, x)$ and $\left(g_{0},-h\right)$ are equal in $G \times \times_{\mathbf{B}} \mathfrak{b}_{0}$. Hence the fiber of $\pi$ at $h$ has two elements. So the same applies for the fiber of $\pi$ at any element of the $G$-invariant cone generated by $h$ since $\pi$ is $G$-equivariant. Hence $K$ is an algebraic extension of degree 2 of $K_{0}$. In particular, it is a Galois extension. Let $A$ be the integral closure of $\mathbb{C}\left[\mathfrak{X}_{\mathfrak{g}}\right]$ in $K$ and let $\mathfrak{X}_{\mathfrak{g}}^{\prime}$ be an affine algebraic variety such that $\mathbb{C}\left[\mathfrak{X}_{\mathfrak{g}}^{\prime}\right]$ is equal to $A$. Then $A$ is stable under the Galois group of the extension $K$ of $K_{0}$ and $\mathbb{C}\left[\mathfrak{X}_{\mathfrak{g}}\right]$ is the subalgebra of invariant elements of this action since $\mathfrak{X}_{\mathfrak{g}}$ is normal. Hence by [E12 (Lemma 1), it is enough to prove that $\mathfrak{X}_{\mathfrak{g}}^{\prime}$ has rational singularities.

The variety $G \times_{\mathbf{B}} \mathfrak{b}_{0}$ is smooth as a vector bundle over $G / \mathbf{B}$. Hence the morphism $\pi$ factors through the canonical morphism from $\mathfrak{X}_{\mathfrak{g}}^{\prime}$ to $\mathfrak{X}_{\mathfrak{g}}$. Let $\pi^{\prime}$ be the morphism from $G \times{ }_{\mathbf{B}} \mathfrak{b}_{0}$ to $\mathfrak{X}_{\mathfrak{g}}^{\prime}$ such that $\pi$ is the compound map of $\pi^{\prime}$ with the canonical morphism from $\mathfrak{X}_{\mathfrak{g}}^{\prime}$ to $\mathfrak{X}_{\mathfrak{g}}$. Then $\left(G \times_{\mathbf{B}} \mathfrak{b}_{0}, \pi^{\prime}\right)$ is a desingularization of $\mathfrak{X}_{\mathfrak{g}}^{\prime}$ since $\pi^{\prime}$ is proper and birational. By the corollary of [He] (Theorem B), for $i \in \mathbb{N}^{*}$, the $i$-th cohomology group $\mathrm{H}^{i}\left(G \times_{\mathbf{B}} \mathfrak{b}_{0}, \mathcal{O}_{G \times \times_{\mathbf{B}} \mathfrak{b}_{0}}\right)$ is equal to zero. Hence by $[\mathbf{H a}]\left(\right.$ Ch. III, Proposition 8.5), for $i \in \mathbb{N}^{*}, \mathrm{R}^{i} \pi_{*}^{\prime}\left(\mathcal{O}_{G \times{ }_{\mathbf{B}} \mathfrak{b}_{0}}\right)=0$ and $\mathfrak{X}_{\mathfrak{g}}^{\prime}$ has rational singularities since $\mathfrak{X}_{\mathfrak{g}}^{\prime}$ is normal.

Remark 4. - The $G$-invariant closed cone generated by a semisimple element of $\mathfrak{g}$ is not a normal variety in general [Ri2 (Proposition 10.1). Nevertheless, whenever $x$ is the semisimple element of a $\mathfrak{s l}_{2}$-triple of $\mathfrak{g}$, the closed cone generated by the regular orbit whose closure contains $x$ is normal and Cohen-Macaulay [Ri2](Proposition 10.3).

2. Principal bicone. - We study in this subsection various properties of the principal bicone.

Definition 3. - The principal bicone of $\mathfrak{g}$ is the subset

$$
\begin{aligned}
\mathcal{X}_{\mathfrak{g}} & :=\left\{(x, y) \in \mathfrak{g} \times \mathfrak{g} \mid P_{x, y} \subset \mathfrak{X}_{\mathfrak{g}}\right\} \\
& =\left\{(x, y) \in \mathfrak{g} \times \mathfrak{g} \mid a x+b y \in \mathfrak{X}_{\mathfrak{g}}, \forall(a, b) \in \mathbb{C}^{2}\right\} .
\end{aligned}
$$


As $\mathcal{N}_{\mathfrak{g}}$, the subset $\mathcal{X}_{\mathfrak{g}}$ is a closed bicone of $\mathfrak{g} \times \mathfrak{g}$, and it is invariant under the actions of $G$ and $\mathrm{GL}_{2}(\mathbb{C})$. For $i=2, \ldots$, rkg $\mathfrak{g}$, we define the elements $q_{i, m, n}$ of $\left(S(\mathfrak{g}) \otimes_{\mathbb{C}} S(\mathfrak{g})\right)^{\mathfrak{g}}$ by the following relation:

$$
q_{i}(a x+b y)=\sum_{m+n=d_{i}} a^{m} b^{n} q_{i, m, n}(x, y),
$$

for $(a, b)$ in $\mathbb{C}^{2}$ and $(x, y)$ in $\mathfrak{g} \times \mathfrak{g}$. For $w$ in $W_{G}(\mathfrak{h})$, we denote by $\mathfrak{b}_{0, w}$ the subspace generated by $w(h)$ and $\mathfrak{u}$. It is worth recalling the following wellknown result for the understanding of the principal bicone.

Lemma 17. - If $x$ is in $\mathfrak{h}$, then the intersection of $G . x$ and $\mathfrak{h}$ is the orbit of $x$ under $W_{G}(\mathfrak{h})$.

Our purpose is to prove that $\mathcal{X}_{\mathfrak{g}}$ is a complete intersection of dimension $3\left(b_{\mathfrak{g}}-\mathrm{rkg}+1\right)$, what we will do in Section 4 .

Lemma 18. - i) The subset $\mathcal{X}_{\mathfrak{g}}$ is the nullvariety of the polynomial functions $q_{i, m, n}$, for $i=2, \ldots$, rkg and $m+n=d_{i}$. In particular, any irreducible component of $\mathcal{X}_{\mathfrak{g}}$ has dimension at least $3\left(\mathrm{~b}_{\mathfrak{g}}-\mathrm{rk} \mathfrak{g}+1\right)$.

ii) The subset $\mathfrak{s} \times \mathfrak{s}$ is contained in $\mathcal{X}_{\mathfrak{g}}$.

iii) For $x$ in $\mathfrak{b},(w(h), x)$ belongs to $\mathcal{X}_{\mathfrak{g}}$ if and only if $x$ is in $\mathfrak{b}_{0, w}$.

Proof. - i) As $\mathfrak{X}_{\mathfrak{g}}$ is the nullvariety of the $q_{i}$ by Lemma 15, (ii), $\mathcal{X}_{\mathfrak{g}}$ is the nullvariety of the polynomial functions $q_{i, m, n}$, for $i=2, \ldots$, rk $\mathfrak{g}$ and $m+n=d_{i}$. As $d_{1}=2, \mathcal{X}_{\mathfrak{g}}$ is the nullvariety in $\mathfrak{g} \times \mathfrak{g}$ of $b_{\mathfrak{g}}-\mathrm{rk} \mathfrak{g}-3$ regular functions, whence the second statement.

ii) Let $T$ be the intersection of $\mathcal{X}_{\mathfrak{g}}$ and $\mathfrak{s} \times \mathfrak{s}$. Then $T$ is a closed, subset of $\mathfrak{s} \times \mathfrak{s}$ invariant under $\mathrm{GL}_{2}(\mathbb{C})$ and $\mathbf{S}$. For any $t$ in $\mathbb{C}, h+$ te belongs to G.h. So $T$ contains $(h, e)$. Hence, by Lemma $9, \operatorname{dim} T \geq 5$ and, $T=T_{5}$ if and only if $\operatorname{dim} T=5$. As $t h$ and $e+t^{2} f$ are in the same $G$-orbit for any $t \in \mathbb{C}^{*},(e, f)$ is in $\mathcal{X}_{\mathfrak{g}}$. So $T$ is equal to $\mathfrak{s} \times \mathfrak{s}$ since $(e, f)$ is not in $T_{5}$.

iii) For any $t$ in $\mathbb{C}^{*}$ and any $x$ in $\mathfrak{u}, t w(h)+x$ belongs to the $G$-orbit of $t w(h)$. Hence $(w(h), t w(h)+x)$ belongs to $\mathcal{X}_{\mathfrak{g}}$. So $\{w(h)\} \times \mathfrak{b}_{0, w}$ is contained in $\mathcal{X}_{\mathfrak{g}}$. Let $\left(x_{1}, x_{2}\right)$ be in $\mathfrak{h} \times \mathfrak{u}$. We suppose that $\left(w(h), x_{1}+x_{2}\right)$ is in $\mathcal{X}_{\mathfrak{g}}$. Then for any $t$ in $\mathbb{C}, w(h)+t x_{1}+t x_{2}$ is in $\mathfrak{X}_{\mathfrak{g}}$. In particular, $x_{1}+x_{2}$ is tangent at $w(h)$ to the cone $\mathfrak{X}_{\mathfrak{g}}$. The tangent space at $w(h)$ of the cone $\mathfrak{X}_{\mathfrak{g}}$ is generated by $w(h)$ and $[w(h), \mathfrak{g}]$. So $x_{1}$ and $w(h)$ are collinear.

3. Smooths points of $\mathcal{X}_{P}$. - As the nilpotent cone $\mathfrak{N}_{\mathfrak{g}}$ is contained in the principal cone $\mathfrak{X}_{\mathfrak{g}}$, the nilpotent bicone $\mathcal{N}_{\mathfrak{g}}$ is contained in the principal bicone $\mathcal{X}_{\mathfrak{g}}$. We introduce now two other varieties "squeezed in between" $\mathcal{N}_{\mathfrak{g}}$ and $\mathcal{X}_{\mathfrak{g}}$. 
Set

$$
\begin{aligned}
\mathcal{Y}_{\mathfrak{g}} & :=\left\{(x, y) \in \mathcal{X}_{\mathfrak{g}} \mid\langle x, y\rangle=0\right\} \\
\mathcal{Z}_{\mathfrak{g}} & :=\left\{(x, y) \in \mathcal{Y}_{\mathfrak{g}} \mid y \in \mathfrak{N}_{\mathfrak{g}}\right\} .
\end{aligned}
$$

Lemma 19. - i) The nilpotent bicone is the nullvariety in $\mathcal{X}_{\mathfrak{g}}$ of $p_{1,2,0}, p_{1,1,1}, p_{1,0,2}$.

ii) The subset $\mathcal{Y}_{\mathfrak{g}}$ is the nullvariety in $\mathcal{X}_{\mathfrak{g}}$ of $p_{1,1,1}$. Moreover, $\mathcal{Y}_{\mathfrak{g}}$ is a $G$ invariant closed bicone and any irreducible component of $\mathcal{Y}_{\mathfrak{g}}$ has dimension at least $3\left(\mathrm{~b}_{\mathfrak{g}}-\mathrm{rkg}\right)+2$.

iii) The subset $\mathcal{Z}_{\mathfrak{g}}$ is the nullvariety in $\mathcal{Y}_{\mathfrak{g}}$ of $p_{1,0,2}$. Moreover, $\mathcal{Z}_{\mathfrak{g}}$ is a $G$ invariant closed bicone and any irreducible component of $\mathcal{Z}_{\mathfrak{g}}$ has dimension at least $3\left(\mathrm{~b}_{\mathfrak{g}}-\mathrm{rk} \mathfrak{g}\right)+1$.

Proof. - i) As $\mathfrak{N}_{\mathfrak{g}}$ is the nullvariety of $p_{1}$ in $\mathfrak{X}_{\mathfrak{g}}$, by Lemma 15 (i), $\mathcal{N}_{\mathfrak{g}}$ is the nullvariety in $\mathcal{X}_{\mathfrak{g}}$ of the 2 -order polarizations of $p_{1}$, that is $p_{1,2,0}, p_{1,1,1}, p_{1,0,2}$.

ii) As $p_{1,1,1}$ is the Killing form, $\mathcal{Y}_{\mathfrak{g}}$ is the nullvariety of $p_{1,1,1}$ in $\mathcal{X}_{\mathfrak{g}}$. Moreover, $\mathcal{Y}_{\mathfrak{g}}$ is a $G$-invariant closed bicone since $p_{1,1,1}$ is $G$-invariant and bihomogeneous. By Lemma 18, (i), any irreducible component of $\mathcal{Y}_{\mathfrak{g}}$ has dimension at least $3\left(\mathrm{~b}_{\mathfrak{g}}-\mathrm{rk} \mathfrak{g}\right)+2$.

iii) By Lemma 15, (i), $\mathfrak{N}_{\mathfrak{g}}$ is the nullvariety of $p_{1}$ in $\mathfrak{X}_{\mathfrak{g}}$. Hence $\mathcal{Z}_{\mathfrak{g}}$ is the nullvariety of $p_{1,0,2}$ in $\mathcal{Y}_{\mathfrak{g}}$. Moreover, $\mathcal{Z}_{\mathfrak{g}}$ is a $G$-invariant closed bicone since $p_{1,0,2}$ is $G$-invariant and bihomogeneous. By (ii), any irreducible component of $\mathcal{Z}_{\mathfrak{g}}$ has dimension at least $3\left(\mathrm{~b}_{\mathfrak{g}}-\mathrm{rk} \mathfrak{g}\right)+1$.

Remark 5. - By Lemma 19, the subsets $\mathcal{Y}_{\mathfrak{g}}$ and $\mathcal{Z}_{\mathfrak{g}}$ inherit a natural structure of scheme.

Let $P$ be a subset of the set of polynomials

$$
\left\{p_{1,2,0}, p_{1,1,1}, p_{1,0,2}, q_{i, m, d_{i}-m}, i=2, \ldots, \mathrm{rkg}, m=0, \ldots, d_{i}\right\} .
$$

We denote by $\mathbb{C}[P]$ the subalgebra of $\mathrm{S}(\mathfrak{g}) \otimes_{\mathbb{C}} \mathrm{S}(\mathfrak{g})$ generated by $P$, we denote by $\sigma_{P}$ the morphism of affine varieties whose comorphism is the canonical injection from $\mathbb{C}[P]$ to $\mathrm{S}(\mathfrak{g}) \otimes_{\mathbb{C}} \mathrm{S}(\mathfrak{g})$ and we denote by $\mathcal{X}_{P}$ the nullvariety of $P$ in $\mathfrak{g} \times \mathfrak{g}$.

Lemma 20. - Let $(x, y)$ be in $\Omega_{\mathfrak{g}}$.

i) The morphism $\sigma_{P}$ is smooth at $(x, y)$.

ii) If $(x, y)$ is in $\mathcal{X}_{P}$, then $(x, y)$ is a smooth point of $\mathcal{X}_{P}$. Moreover, the unique irreducible component of $\mathcal{X}_{P}$ which contains $(x, y)$ has dimension $2 \operatorname{dim} \mathfrak{g}-|P|$.

Proof. - i) Let $\rho$ be the morphism whose comorphism is the canonical injection from $\mathbb{C}[P]$ to the subalgebra generated by the polynomials $p_{i, m, d_{i}-m}$ 
where $i=1, \ldots, \operatorname{rkg}$ and $m=0, \ldots, d_{i}$. Then $\sigma_{P}$ is equal to $\rho \circ \sigma$, where $\sigma$ is the morphism introduced in Subsection 4. As $q_{i, m, d_{i}-m}$ is a linear combination of $p_{i, m, d_{i}-m}$ and homogeneous elements in the subalgebra generated by $p_{1,2,0}$, $p_{1,1,1}, p_{1,0,2}$, for $i=2, \ldots$, rk $\mathfrak{g}$ and $m=0, \ldots, d_{i}$, the morphism $\rho$ is smooth. Then by Proposition 13, $\sigma_{P}$ is a smooth morphism at $(x, y)$.

ii) We suppose that $(x, y)$ is in $\mathcal{X}_{P}$. By definition, $\mathcal{X}_{P}$ is the fiber at 0 of the morphism $\sigma_{P}$. So by (i), $(x, y)$ is a smooth point of $\mathcal{X}_{P}$ and the codimension in $\mathfrak{g} \times \mathfrak{g}$ of the irreducible component of $\mathcal{X}_{P}$ which contains $(x, y)$ is equal to the dimension of $\mathbb{C}[P]$. By Proposition 13 , the polynomials $p_{i, m, d_{i}-m}$ are algebraically independent for $i=1, \ldots, \operatorname{rkg}$ and $m=0, \ldots, d_{i}$. So the elements of $P$ are algebraically independent since $q_{i, m, d_{i}-m}$ is a linear combination of $p_{i, m, d_{i}-m}$ and homogeneous elements in the subalgebra generated by $p_{1,2,0}, p_{1,1,1}, p_{1,0,2}$, for $i=2, \ldots$, rk $\mathfrak{g}$ and $m=0, \ldots, d_{i}$. Hence the irreducible component of $\mathcal{X}_{P}$ containing $(x, y)$ has dimension $2 \operatorname{dim} \mathfrak{g}-|P|$.

The principal bicone $\mathcal{X}_{\mathfrak{g}}$ has irreducible components of the expected dimension as the following proposition shows. Actually this proposition will be not used in the other sections.

Proposition 21. - Let $w$ be in $W_{G}(\mathfrak{h})$. There is an unique irreducible component $\chi(w)$ of $\mathcal{X}_{\mathfrak{g}}$ containing $(w(h), e)$ and $\chi(w)$ satisfies the following properties:

1) $\chi(w)$ has dimension $3\left(\mathrm{~b}_{\mathfrak{g}}-\mathrm{rk} \mathfrak{g}+1\right)$,

2) $\chi\left(w w_{0}\right)$ is equal to $\chi(w)$,

3) $\chi(w)$ contains $\left(w_{0} w(h), f\right),(e, w(h)),\left(f, w_{0} w(h)\right)$.

Moreover, $\chi(w)$ contains $\mathfrak{s} \times \mathfrak{s}$ if $w=1$ or $w=w_{0}$.

Proof. - Let $P$ be the subset $\left\{q_{i, m, d_{i}-m}, i=2, \ldots, \mathrm{rkg}, m=0, \ldots, d_{i}\right\}$. Then $\mathcal{X}_{P}$ is equal to $\mathcal{X}_{\mathfrak{g}}$ by Lemma 18, (i). By Lemma 18, (iii), $\mathcal{X}_{\mathfrak{g}}$ contains $(w(h), e)$ and by Lemma 6, (i), $(w(h), e)$ belongs to $\Omega_{\mathfrak{g}}$. Hence by Lemma 20, (ii), $(w(h), e)$ is a smooth point of $\mathcal{X}_{\mathfrak{g}}$. So there is an unique irreducible component of $\mathcal{X}_{\mathfrak{g}}$ which contains $(w(h), e)$. Moreover, this component has dimension $3\left(\mathrm{~b}_{\mathfrak{g}}-\mathrm{rk} \mathfrak{g}+1\right)$. Let us denote it by $\chi(w)$. Then $\chi(w)$ satisfies condition $(1)$. As $w w_{0}(h)$ is equal to $-w(h)$, by Lemma 14 $\chi(w)$ contains $\left(w w_{0}(h), e\right)$ since $\chi(w)$ is a bicone. Moreover, by Lemma 14, $\chi(w)$ contains $\left(w_{0} w(h), f\right)$ since $\chi(w)$ is $G$-invariant and $g_{0} \cdot(w(h), e)$ is equal to $\left(w_{0} w(h), f\right)$. As $\chi(w)$ is also $\mathrm{GL}_{2}(\mathbb{C})$-invariant, it is invariant under the involution $(x, y) \mapsto(y, x)$. So $\chi(w)$ contains $(e, w(h))$ and $\left(f, w_{0} w(h)\right)$.

By Lemma 18, (ii), $\mathfrak{s} \times \mathfrak{s}$ is contained in $\mathcal{X}_{\mathfrak{g}}$. Hence there exists an irreducible component of $\mathcal{X}_{\mathfrak{g}}$ which contains $\mathfrak{s} \times \mathfrak{s}$. But $\mathfrak{s} \times \mathfrak{s}$ contains $(h, e)$, which is a smooth point of $\mathcal{X}_{\mathfrak{g}}$. So $\chi(w)$ contains $\mathfrak{s} \times \mathfrak{s}$ if $w(h)$ is colinear to $h$, that is to say $w=1$ or $w=w_{0}$. 
Remark 6. - When $\mathfrak{g}$ is simple of type $B_{\ell}, C_{\ell}, D_{2 \ell}, E_{7}, E_{8}, F_{4}$ or $G_{2}, w_{0}$ is equal to -1 . So in these cases, for any $w$ in $W_{G}(\mathfrak{h}), \chi(w)$ contains $(w(h), f)$ and $(f, w(h))$ since $\chi(w)$ is a bicone. A quick computation shows that $\mathcal{X}_{\mathfrak{g}}$ precisely has $\left|W_{G}(\mathfrak{h})\right| / 2$ irreducible components when $\mathfrak{g}$ is equal to $\mathfrak{s l}_{n}(\mathbb{C})$ for $n=2,3$.

4. Proof of Corollary 23, - The goal of this subsection is to prove Corollary 23. This corollary will be crucial for the study of the dimension of $\mathcal{X}_{\mathfrak{g}}$ (see Section 4).

Lemma 22. - Let $\mathcal{Z}$ be an irreducible closed subset of $\mathcal{Z}_{\mathfrak{g}}$ satisfying the two following conditions:

1) $\varpi_{1}(\mathcal{Z})$ contains a non zero semisimple element of $\mathfrak{g}$,

2) $\varpi_{2}(\mathcal{Z})$ contains a regular nilpotent element of $\mathfrak{g}$.

Then $\mathcal{Z}$ has a nonempty intersection with $\Omega_{\mathfrak{g}}$.

Proof. - By Condition (1), the subset $\mathcal{Z}_{1}$ of elements $(x, y)$ in $\mathcal{Z}$ such that $x$ is a non zero semisimple element of $\mathfrak{g}$ is a nonempty open subset of $\mathcal{Z}$ since the subset of semisimple elements of $\mathfrak{g}$ which belongs to $\mathfrak{X}_{\mathfrak{g}}$ is an open dense subset of $\mathfrak{X}_{\mathfrak{g}}$. On the other hand, by Condition (2), the subset $\mathcal{Z}_{2}$ of elements $(x, y)$ in $\mathcal{Z}$ such that $y$ is a regular nilpotent element of $\mathfrak{g}$ is a nonempty open subset of $\mathcal{Z}$ since the subset of regular nilpotent elements is an open dense subset of $\mathfrak{N}_{\mathfrak{g}}$. Then the intersection of $\mathcal{Z}_{1}$ and $\mathcal{Z}_{2}$ is nonempty subset since $\mathcal{Z}$ is irreducible. Let $(x, y)$ be in the intersection $\mathcal{Z}_{1}$ and $\mathcal{Z}_{2}$. Suppose that $(x, y)$ doesn't belong to $\Omega_{\mathfrak{g}}$. We expect a contradiction. Then there exists $t \in \mathbb{C}$ such that $t x+y$ is not a regular element of $\mathfrak{g}$. Indeed $x$ is regular as non zero semisimple element of $\mathfrak{X}_{\mathfrak{g}}$. As $(x, y)$ belongs to the principal bicone, $t x+y$ is a non regular element of $\mathfrak{X}_{\mathfrak{g}}$, whence

$$
\langle t x+y, t x+y\rangle=0,
$$

by Lemma 15, (iii). But the left hand side of this equality is equal to $t^{2}\langle x, x\rangle$ since $(x, y)$ belongs to $\mathcal{Z}_{\mathfrak{g}}$. Hence $t=0$ since for any non zero semisimple element $z$ in $\mathfrak{X}_{\mathfrak{g}},\langle z, z\rangle \neq 0$. This contradicts the fact that $y$ is regular. So $\mathcal{Z}$ has a nonempty intersection with $\Omega_{\mathfrak{g}}$.

Corollary 23. - Let $\mathcal{X}$ be an irreducible component of $\mathcal{X}_{\mathfrak{g}}$. We denote by $\mathcal{X}^{\prime}$ the intersection of $\mathcal{X}$ and $\mathcal{Z}_{\mathfrak{g}}$. Let us suppose that $\mathcal{X}^{\prime}$ has an irreducible component $\mathcal{Z}$ satisfying the two following conditions:

1) $\varpi_{1}(\mathcal{Z})$ is equal to $\mathfrak{X}_{\mathfrak{g}}$,

2) $\varpi_{2}(\mathcal{Z})$ is equal to $\mathfrak{N}_{\mathfrak{g}}$.

Then $\mathcal{X}^{\prime}$ and $\mathcal{X}$ have a non empty intersection with $\Omega_{\mathfrak{g}}$. In particular, $\mathcal{X}$ has dimension $3\left(\mathrm{~b}_{\mathfrak{g}}-\mathrm{rkg}+1\right)$. 
Proof. - By Lemma 22, $\mathcal{Z}, \mathcal{X}^{\prime}$ and $\mathcal{X}$ have a nonempty intersection with $\Omega_{\mathfrak{g}}$. Then, by Lemma 20, (ii), $\mathcal{X}$ has dimension $3\left(\mathrm{~b}_{\mathfrak{g}}-\mathrm{rk} \mathfrak{g}+1\right)$.

\section{Jet schemes and motivic integration}

We plan to study in Section 4 the dimensions of the nilpotent bicone and the principal bicone via motivic integration arguments. In view of this work, we start this section by some basics on motivic integration.

1. Jet schemes. - Let us first review the definition and properties of jet schemes. Let $X$ be a complex algebraic variety. For $m \in \mathbb{N}$, the $m$-order jet scheme $J_{m}(X)$ of $X$ is the scheme whose closed points over $x \in X$ are morphisms

$$
\mathcal{O}_{X, x} \longrightarrow \mathbb{C}[t] / t^{m+1}
$$

Thus, the $\mathbb{C}$-valued points of $J_{m}(X)$ are in natural bijection with the $\mathbb{C}[t] / t^{m+1}$-valued points of $X$. In particular, there are canonical isomorphisms $J_{0}(X) \simeq X$ and $J_{1}(X) \simeq \mathrm{T} X$, where $\mathrm{T} X$ is the total tangent space of $X$. The canonical morphisms

$$
\pi_{l, m}: J_{l}(X) \longrightarrow J_{m}(X)
$$

with $l \geq m$, obtained by truncation, induce a projective system $\left(J_{m}(X), \pi_{l, m}\right)$. The space of the arcs

$$
J_{\infty}(X):=\operatorname{proj} \lim _{m} J_{m}(X)
$$

is the projective limit of the system $\left(J_{m}(X), \pi_{l, m}\right)$. Denote by $\pi_{\infty, m}$ the canonical morphism

$$
\pi_{\infty, m}: J_{\infty}(X) \longrightarrow J_{m}(X),
$$

for $m \in \mathbb{N}$, obtained by truncation, too. For $\nu$ in $J_{\infty}(X)$ and $\varphi$ a regular function on $X$, we denote by $\operatorname{ord}(\nu \circ \varphi)$ the order of the serie $\nu \circ \varphi$. If $\mathcal{I}$ is an ideal of $\mathcal{O}_{X}$, we denote by $\operatorname{ord}(\mathcal{I}, \nu)$ the smallest integer $\operatorname{ord}(\nu \circ \varphi)$, where $\varphi$ runs through the ideal $\mathcal{I}_{\pi_{\infty, 0}(\nu)}$ generated by $\mathcal{I}$ in the local ring $\mathcal{O}_{X, \pi_{\infty, 0}(\nu)}$. The function $F_{\mathcal{I}}: \nu \mapsto \operatorname{ord}(\mathcal{I}, \nu)$ is semialgebraic by [DL2](Theorem 2.1). As a matter of fact, for $m \in \mathbb{N}$, we can define an analogous function from $J_{m}(X)$ to $\{1, \ldots, m\}$ that we denote by the same symbol. When $\mathcal{I}$ is the ideal of definition of a closed subset $Z$ of $X$, we rather denote by $F_{Z}$ the function $F_{\mathcal{I}}$. In particular, if $\mathcal{I}$ is the ideal of definition of a divisor $D$ of $X$, we denote by $F_{D}$ the function $F_{\mathcal{I}}$.

In [Mu], M. Mustaţă proves the following result, first conjectured by David Eisenbud and Edward Frenkel: 
Theorem 24 (Mustaţă). - If $X$ is locally a complete intersection variety, then $J_{m}(X)$ is irreducible of dimension $\operatorname{dim} X(m+1)$ for all $m \geq 1$ if and only if $X$ has rational singularities.

Remark 7. - The nilpotent cone $\mathfrak{N}_{\mathfrak{g}}$ of the reductive Lie algebra $\mathfrak{g}$ is a complete intersection and by $[\mathbf{H e}$ (Theorem A), it has rational singularities. Likewise, by Corollary 16, the principal cone $\mathfrak{X}_{\mathfrak{g}}$ of $\mathfrak{g}$ is a complete intersection and has rational singularities. As a consequence, Theorem 24 can be applied to $\mathfrak{N}_{\mathfrak{g}}$ or $\mathfrak{X}_{\mathfrak{g}}$. In the appendix of $[\mathbf{M u}]$, D. Eisenbud and E. Frenkel apply Theorem 24 to $\mathfrak{N}_{\mathfrak{g}}$ to extend Kostant's results in the setting of jet schemes.

2. Motivic integration. - Recall now some facts about the theory of motivic integration. The construction of motivic integrals for smooth spaces is due to Kontsevich [Kon and was generalized by Denef and Loeser to singular spaces in DL2 and [DL1]. Further in the paper, we will only need of the Hodge realization of motivic integrals on the arcs of a smooth variety. We refer to $\mathbf{B a}, \overline{\mathbf{D L}}$ and $\mathbf{L}$ ] for more explanations, definitions and proofs (see also $\mathbf{C r}$ for an introduction).

Let $\mathcal{V}$ be the category of complex algebraic varieties. Denote by $\mathrm{K}_{0}(\mathcal{V})$ the Grothendieck ring of $\mathcal{V}$ and denote by $[X]$ the class in $\mathrm{K}_{0}(\mathcal{V})$ of an element $X$ in $\mathcal{V}$. The map $X \mapsto[X]$ naturally extends to the category of constructible subsets of algebraic varieties. Let $\mathbb{L}$ be the class of $\mathbb{A}^{1}$ in $K_{0}(\mathcal{V})$ and let $\mathbf{M}$ be the localization $\mathrm{K}_{0}(\mathcal{V})\left[\mathbb{L}^{-1}\right]$. For $m$ in $\mathbb{Z}$, we denote by $F^{m} \mathbf{M}$ the subgroup of $\mathbf{M}$ generated by the elements $[X] \mathbb{L}^{-r}$, where $r-\operatorname{dim} X \geq m$. Then we get a decreasing filtration of the ring $\mathbf{M}$ and we denote by $\widehat{\mathbf{M}}$ its separated completion.

Let $X$ be an algebraic variety of pure dimension $d$. A subset $A$ in $J_{\infty}(X)$ is called a cylinder if $A$ is a finite union of fibers of $\pi_{\infty, m}: J_{\infty}(X) \longrightarrow J_{m}(X)$, for $m \in \mathbb{N}$. If $A \in J_{\infty}(X)$ is a cylinder, we say that $A$ is stable at level $m \in \mathbb{N}$ if, for any $n \geq m$, the map $\pi_{\infty, n+1}\left(J_{\infty}(X)\right) \longrightarrow \pi_{\infty, n}\left(J_{\infty}(X)\right)$ is a piecewise trivial fibration over $\pi_{\infty, n}(A)$ with fiber $\mathbb{A}^{d}$. When $X$ is smooth, any cylinder is stable and the later additional condition is superfluous.

\section{Proposition 25 ([DL2] (Definition-Proposition 3.2))}

There is a well-defined subalgebra $\mathbf{B}_{X}$ of the Boolean algebra of subsets of $J_{\infty}(X)$ which contains the cylinders and a map $\mu_{X}$ from $\mathbf{B}_{X}$ to $\widehat{\mathbf{M}}$ satisfying the following properties:

1) if $A \in \mathbf{B}_{X}$ is stable at level $m$, then $\mu_{X}(A)=\left[\pi_{\infty, m}(A)\right] \mathbb{L}^{-(m+1) d}$,

2) if $Y$ is a closed subvariety in $X$ of dimension strictly smaller than $d$, then for any $A$ in $\mathbf{B}_{X}$, contained in $J_{\infty}(Y), \mu_{X}(A)=0$, 
3) let $A_{1}, A_{2}, \ldots$ be a sequence of elements in $\mathbf{B}_{X}$ whose union $A$ is in $\mathbf{B}_{X}$, then the sequence $\mu_{X}\left(A_{1}\right), \mu_{X}\left(A_{2}\right), \ldots$ converges to $\mu_{X}(A)$ in $\widehat{\mathbf{M}}$,

4) if $A$ and $B$ are in $\mathbf{B}_{X}, A$ is contained in $B$ and $\mu_{X}(B)$ belongs to the closure of $F^{m} \mathbf{M}$ in $\widehat{\mathbf{M}}$, then $\mu_{X}(A)$ belongs to the closure of $F^{m} \mathbf{M}$ in $\widehat{\mathbf{M}}$.

For $A$ in $\mathbf{B}_{X}$ and $\psi: A \longrightarrow \mathbb{Z} \cup\{\infty\}$ a function such that $\psi^{-1}(s) \in \mathbf{B}_{X}$ for any $s \in \mathbb{Z} \cup\{\infty\}$ and $\mu_{X}\left(\psi^{-1}(\infty)\right)=0$, we can set

$$
\int_{A} \mathbb{L}^{-\psi} \mathrm{d} \mu_{X}:=\sum_{s \in \mathbb{Z}} \mu_{X}\left(\psi^{-1}(s)\right) \mathbb{L}^{-s}
$$

in $\widehat{\mathbf{M}}$, whenever the right hand side converges in $\widehat{\mathbf{M}}$. In this case, we say that $\mathbb{L}^{-\psi}$ is integrable on $A$.

Recall that the dimension of a non irreducible variety is the maximal dimension of its irreducible components. Let $u$ and $v$ be two indeterminate variables. For any smooth projective variety $X$, the Hodge-Deligne polynomial of $X$ is the element of $\mathbb{Z}[u, v]$

$$
h(X):=\sum_{k \in \mathbb{N}} \sum_{\substack{(p, q) \in \mathbb{N}^{2} \\ p+q=k}}(-1)^{k} h_{p, q}\left(\mathrm{H}_{c}^{k}(X ; \mathbb{C})\right) u^{p} v^{q},
$$

where $h_{p, q}\left(\mathrm{H}_{c}^{k}(X ; \mathbb{C})\right)$ are the Hodge-Deligne numbers of $X$. The map $h$ factors through the ring $\mathrm{K}_{0}(\mathcal{V})$. So we have a morphism $h$ from $\mathrm{K}_{0}(\mathcal{V})$ to $\mathbb{Z}[u, v]$ such that $h(X)$ is equal to $h([X])$ for any smooth projective variety. In particular, $h(\mathbb{L})$ is equal to $u v$. What is important for us is that $h([X])$ is a polynomial whose highest degree term is $c(u v)^{\operatorname{dim} X}$, where $c$ is the number of irreducible components of $X$ of dimension $\operatorname{dim} X$. By continuity, the morphism $h$ uniquely extends to a morphism from $\widehat{\mathbf{M}}$ to the ring $\mathbb{Z}[u, v]\left[\left[u^{-1}, v^{-1}\right]\right]$. The compound map $h \circ \mu_{X}$, which is now well-defined, is the Hodge realization of the motivic measure.

3. Some technical results. - Let $V$ be a finite dimensional vector space. We study in this subsection various properties of specific subsets in $J_{\infty}(V)$.

For $(x, y)$ in $V \times V$, we denote by $\nu_{x, y}$ the arc $t \mapsto x+t y$ of $J_{\infty}(V)$. Let $m$ be in $\mathbb{N}^{*}$. We denote by $\nu_{x, y, m}$ the image of $\nu_{x, y}$ by the canonical projection $\pi_{\infty, m}^{V}$ from $J_{\infty}(V)$ to $J_{m}(V)$. Let $\mathbf{K}$ be a connected closed subgroup of GL $(V)$. The $\mathbf{K}$-action on $V$ extends to a $\mathbf{K}$-action on $J_{m}(V)$ which is compatible with the canonical projections $\pi_{\infty, m}^{V}$. Let $X$ be a $\mathbf{K}$-invariant irreducible closed cone in $V$. We suppose that $X$ is a complete intersection in $V$ with rational singularities and we suppose that $X$ is a finite union of $\mathbf{K}$-orbits. Let $N$ be the dimension of $X$ and let $r$ be the codimension of $X$ in $V$. 
We use now techniques developed in $\mathbf{M u}$ (Theorem 3.2) in order to prove Proposition 28. We denote by $Z$ the union of $\mathbf{K}$-orbits in $X$ which are not of maximal dimension. Let $B_{X}$ be the blowing up of $V$ whose center is $X$ and let

$$
\tau: B_{X} \longrightarrow V
$$

be the morphism of the blowing-up. As $X$ is a complete intersection in $V$, $\tau^{-1}(X)$ is an integral divisor on $B_{X}$ and is locally a complete intersection. Moreover, there exists a regular action of $\mathbf{K}$ on $B_{X}$ for which $\tau$ is a $\mathbf{K}$ equivariant morphism. By the theorem of embedded desingularization of $\mathrm{Hi}$ ronaka [Hi], there exists a desingularization $(Y, \tilde{\tau})$ of $B_{X}$ such that $(\tau \circ \tilde{\tau})^{-1}(X)$ is a divisor with normal crossings. Moreover, we can find $(Y, \tilde{\tau})$ such that there exists a regular action of $\mathbf{K}$ on $Y$ for which $\tilde{\tau}$ is an equivariant morphism, and $(\tau \circ \tilde{\tau})^{-1}(X)$ is a $\mathbf{K}$-invariant divisor. Set $\gamma:=\tau \circ \tilde{\tau}$. Denoting by $E_{1}, \ldots, E_{t}$ the irreducible components of $\gamma^{-1}(X)$, we can assume that the following conditions are fulfilled:

a) $E_{1}$ is the only prime divisor dominating $X$,

b) the divisor $\gamma^{-1}(X)$ is equal to $\sum_{i=1}^{t} a_{i} E_{i}$,

c) the discrepancy $W$ of $\gamma$ is equal to $\sum_{i=1}^{t} b_{i} E_{i}$,

d) $a_{1}$ is equal to 1 and $b_{1}+1$ is equal to $r$,

e) $\gamma^{-1}(Z)$ is contained in the union of $E_{2}, \ldots, E_{t}$,

since $\tau^{-1}(X)$ is an integral divisor on $B_{X}$ and $\mathbf{K}$ has finitely many orbits in $X$. By Condition (b), $E_{1}, \ldots, E_{t}$ are $\mathbf{K}$-invariant. Moreover, by Condition (e), $Z$ is the image by $\gamma$ of the union of $E_{2}, \ldots, E_{t}$. So there exist $c_{2}, \ldots, c_{t} \in \mathbb{N}$, such that $\gamma^{-1}(Z)=\sum_{i=2}^{t} c_{i} E_{i}$. For $m \in \mathbb{N} \cup\{\infty\}$, let

$$
\gamma_{m}: J_{m}(Y) \longrightarrow J_{m}(V)
$$

be the morphism induced by $\gamma$. In the remainder of this subsection, for $m \in \mathbb{N} \cup\{\infty\}$ and $n \in \mathbb{N}$ with $m \geq n, \pi_{m, n}$ refers to the canonical morphism $J_{m}(Y) \longrightarrow J_{n}(Y)$.

As $X$ has rational singularities, the canonical injection from its canonical module to its dualizing module is an isomorphism by [Fl (Satz 1.1). But its canonical module is locally free of rank 1 since $X$ is Gorenstein as a complete intersection in $V \underline{\mathbf{B r}}$ (Theorem 3.3.7 and Proposition 3.1.20). So $X$ has canonical singularities. Moreover, by $\mathbf{M u}$ (Theorem 2.1), $b_{i} \geq r a_{i}$ for $i=1, \ldots, t$. Then by the above Condition (d), we have $b_{i} \geq\left(b_{1}+1\right) a_{i}$, for $i=2, \ldots, t$. For $k \in \mathbb{N}$, for $J$ a nonempty subset of $\{1, \ldots, t\}$ and for $J^{\prime}$ a nonempty subset of $J$, we denote by $\psi_{k, J, J^{\prime}}$ the affine functional on $\mathbb{Q}^{|J|}$

$$
\left(\alpha_{i}, i \in J\right) \longmapsto\left(N-\left|J^{\prime}\right|\right)(m+1)+\sum_{i \in J}\left(b_{i}+1\right) \alpha_{i}-\sum_{i \in J \backslash J^{\prime}} \alpha_{i}+k \sum_{i \in J} c_{i} \alpha_{i} .
$$


For $p \in \mathbb{N}^{*}$, we set

$$
\begin{aligned}
A_{J, J^{\prime}, p}:=\left\{\left(\alpha_{i}, i \in J\right) \in \mathbb{N}^{|J|} \mid\right. & \sum_{i \in J} a_{i} \alpha_{i} \geq p \text { and } \alpha_{i} \geq m+1, i \in J^{\prime} \\
& \text { and } \left.1 \leq \alpha_{i} \leq m, i \in J \backslash J^{\prime}\right\}
\end{aligned}
$$

and we denote by $\nu_{k, J, J^{\prime}, p}$ the minimal value of $\psi_{k, J, J^{\prime}}$ on $A_{J, J^{\prime}, p}$. The two assertions of Lemma 26 are straightforward from the definitions and the preceding inequalities: $b_{i} \geq\left(b_{1}+1\right) a_{i}$, for $i=2, \ldots, t$. Their verifications are left to the reader.

Lemma 26. - For $k \in \mathbb{N}^{*}, p \geq m+1, J \subseteq\{1, \ldots, t\}$ and $J^{\prime}$ a nonempty subset of $J$, we have:

i) $\nu_{0, J, J^{\prime}, p}=p\left(b_{1}+1\right)+(N-1)(m+1)=\nu_{k, J, J^{\prime}, p}$.

ii) $\nu_{k,\{1\},\{1\}, p} \leq \nu_{k, J, J^{\prime}, p}$.

For $J \subseteq\{1, \ldots, t\}$, we set:

$$
E_{J}^{0}:=\cap_{i \in J} E_{i} \backslash \cup_{i \notin J} E_{i} .
$$

Let $m$ be in $\mathbb{N}^{*}$ and let $S$ be a nonempty constructible subset of $J_{m}(V)$. For $\alpha=\left(\alpha_{i}\right)_{i \in J} \in\left(\mathbb{N}^{*}\right)^{|J|}$, we denote by $J_{\alpha}$ the subset of elements $i \in J$ such that $\alpha_{i} \geq m+1$ and we set:

$$
T_{\alpha}:=\left\{\nu \in \pi_{m, 0}^{-1}\left(E_{J}^{0}\right) \cap \gamma_{m}^{-1}(S) \mid F_{E_{i}}(\nu)=\alpha_{i}, i \in J \backslash J_{\alpha}\right\} .
$$

For $q \in \mathbb{N} \cup\{\infty\}$, with $q \geq m+1$, we set:

$$
S_{\alpha}^{q}:=\left\{\nu \in \pi_{q, 0}^{-1}\left(E_{J}^{0}\right) \cap\left(\gamma_{m} \circ \pi_{q, m}\right)^{-1}(S) \mid F_{E_{i}}(\nu)=\alpha_{i}, i \in J\right\} .
$$

Lemma 27. - Fix $J \subseteq\{1, \ldots, t\}, q \geq m+1$ and $\alpha=\left(\alpha_{i}\right)_{i \in J} \in\left(\mathbb{N}^{*}\right)^{|J|}$. Suppose that $S_{\alpha}^{q}$ is a nonempty set. Then $S_{\alpha}^{q}$ is a locally trivial fibration over $T_{\alpha}$ whose fiber is isomorphic to

$$
\mathbb{C}^{(q-m)\left(N-\left|J_{\alpha}\right|\right)} \times\left(\mathbb{C}^{*}\right)^{\left|J_{\alpha}\right|} \times \mathbb{C}^{q\left|J_{\alpha}\right|-\sum_{i \in J_{\alpha}} \alpha_{i}} .
$$

Proof. - We follow the proof of $[\mathbf{C r}$ (Proposition 2.5). Since the divisor $\gamma^{-1}(X)=\sum_{i=1}^{t} a_{i} E_{i}$ on $Y$ has only simple normal crossing, for any $y$ in $Y$, there exists a neighborhood $U$ of $y$ in $Y$ with global coordinates $z_{1}, \ldots, z_{N}$ on $U$ for which a local defining equation for $\gamma^{-1}(X)$ is given by

$$
g=z_{1}^{a_{1}} \cdots z_{j_{y}}^{a_{j_{y}}}
$$

for some $j_{y} \leq N$. We cover $Y=\bigcup U$ by finitely many charts on which $\gamma^{-1}(X)$ has a local equation of the form (4), and we lift to cover $J_{q}(Y)=\bigcup \pi_{q, 0}^{-1}(U)$. 
Hence $S_{\alpha}^{q}$ is covered by the subsets

$$
U_{\alpha}^{q}:=\bigcap_{i \in J} F_{E_{i}}^{-1}\left(\alpha_{i}\right) \cap \pi_{q, 0}^{-1}(U) \cap \pi_{q, m}^{-1}\left(\gamma_{m}^{-1}(S)\right) .
$$

As $\alpha_{i} \geq 1$, the subset $U_{\alpha}^{q}$ is contained in $\pi_{q, 0}^{-1}\left(E_{J}^{0}\right)$. Thus, the subsets $U_{\alpha}^{q}$ are open subsets of $S_{\alpha}^{q}$. Let $U_{\alpha}^{q}$ be such an open subset of $S_{\alpha}^{q}$ which is nonempty. If $J$ is not contained in $\left\{1, \ldots, j_{y}\right\}$ then $E_{J}^{0} \cap U$ is empty, and so $U_{\alpha}^{q}$. So $J$ is contained in $\left\{1, \ldots, j_{y}\right\}$.

For $\nu \in \pi_{q, 0}^{-1}(U)$, we can view $\nu$ as an $N$-tuple $\left(f_{1}(z), \ldots, f_{N}(z)\right)$ of polynomials of degree at most $q$ with zero constant term. We continue to argue as in the proof of $\left[\mathbf{C r}\right.$ (Proposition 2.5). We see that $\nu \in F_{E_{i}}^{-1}\left(\alpha_{i}\right)$ if and only if the truncation of $f_{i}(z)$ to degree $\alpha_{i}$ is of the form $c_{\alpha_{i}} z^{\alpha_{i}}$ where $c_{\alpha_{i}}$ is different from 0 . Then we obtain $N-|J|$ polynomials of degree $q$ with zero constant term, and, for each $j \in J$, a polynomial of the form

$$
f_{j}(z)=0+\cdots+0+c_{\alpha_{j}} z^{\alpha_{j}}+c_{\alpha_{j+1}} z^{\alpha_{j+1}}+\cdots+c_{\alpha_{q}} z^{\alpha_{q}}
$$

with $c_{\alpha_{j}} \in \mathbb{C}^{*}$ and $c_{k} \in \mathbb{C}$, for $k>j$. So, when we cut the $m$ first terms of each polynomial $f_{j}(z)$, the space of all such $N$-tuples so obtained is isomorphic to

$$
\mathbb{C}^{(q-m)\left(N-\left|J_{\alpha}\right|\right)} \times\left(\mathbb{C}^{*}\right)^{\left|J_{\alpha}\right|} \times \mathbb{C}^{q\left|J_{\alpha}\right|-\sum_{i \in J_{\alpha}} \alpha_{i}} .
$$

As a consequence, $U_{\alpha}^{q}$ is isomorphic to

$$
\left(\pi_{m, 0}^{-1}(U) \cap T_{\alpha}\right) \times \mathbb{C}^{(q-m)\left(N-\left|J_{\alpha}\right|\right)} \times\left(\mathbb{C}^{*}\right)^{\left|J_{\alpha}\right|} \times \mathbb{C}^{q\left|J_{\alpha}\right|-\sum_{i \in J_{\alpha}} \alpha_{i}},
$$

whence the lemma.

Let $S$ be a constructible subset of $J_{m}(V)$. For $p \geq m+1$, we set

$$
S_{\infty, m, p}:=\left\{\nu \in\left(\pi_{\infty, m}^{V}\right)^{-1}(S) \mid F_{X}(\nu) \geq p\right\} .
$$

Then, for $k>0$, we set

$$
\mathbf{I}_{m, p, k}:=h\left(\int_{S_{\infty, m, p}} \mathbb{L}^{-k F_{Z}(\nu)} \mathrm{d} \mu_{V}(\nu)\right) .
$$

Proposition 28. - Let $m, k$ be in $\mathbb{N}^{*}$ and let $S$ be a constructible subset of $J_{m}(V)$. We suppose that the image by $\pi_{m, 0}^{V}$ of $S$ is dense in $X$ and we suppose that $S$ is $\mathbf{K}$-invariant.

i) We suppose that the image by $\pi_{m, 0}^{V}$ of any irreducible component of maximal dimension of $S$ is dense in $X$. Then the highest degree terms of $\mathbf{I}_{m, p, k}$ does not depend on $k$ for $p$ big enough.

ii) We suppose that the highest degree terms of $\mathbf{I}_{m, p, k}$ does not depend on $k$ for $p$ big enough. Then, for $p$ big enough, the highest degree terms of $h \circ \mu_{V}\left(S_{\infty, m, p}\right)$ only depend on the irreducible components of $S$ whose image by $\pi_{m, 0}^{V}$ is dense in $X$. 
Proof. - We apply the transformation rule for the motivic integrals to $\mathbf{I}_{m, p, k}$. Namely, by DL2](Lemma 3.3), we have

$$
\mathbf{I}_{m, p, k}=h\left(\int_{\gamma_{\infty}^{-1}\left(S_{\infty, m, p}\right)} \mathbb{L}^{-F_{W}(\nu)-k \sum_{i=2}^{t} c_{i} F_{E_{i}}(\nu)} \mathrm{d} \mu_{Y}(\nu)\right) .
$$

For $J \subseteq\{1, \ldots, t\}$ and $\alpha=\left(\alpha_{i}\right)_{i \in J} \in\left(\mathbb{N}^{*}\right)^{|J|}, S_{\alpha}^{\infty}$ is the inverse image by $\pi_{\infty, q}$ of $S_{\alpha}^{q}$, for any $q \geq m+1$. So, by Proposition 25, (1), $h \circ \mu_{Y}\left(S_{\alpha}^{\infty}\right)=h\left(\left[S_{\alpha}^{q}\right]\right)(u v)^{-N(q+1)}$. In addition, by Lemma 27, the subset $S_{\alpha}^{q}$ is a locally trivial fibration over $T_{\alpha}$ whose fiber is isomorphic to

$$
\mathbb{C}^{(q-m)\left(N-\left|J_{\alpha}\right|\right)} \times\left(\mathbb{C}^{*}\right)^{\left|J_{\alpha}\right|} \times \mathbb{C}^{q\left|J_{\alpha}\right|-\sum_{i \in J_{\alpha}} \alpha_{i}} .
$$

Then we deduce from $\mathbf{C r}$ (Theorem 3.2, (iii)) the relation:

$$
\begin{aligned}
h \circ \mu_{Y}\left(S_{\alpha}^{\infty}\right)= & h\left(\left[T_{\alpha}\right]\right)(u v-1)^{\left|J_{\alpha}\right|} \\
& (u v)^{-\left|J_{\alpha}\right|-\left(N-\left|J_{\alpha}\right|\right)(m+1)-\sum_{i \in J_{\alpha}} \alpha_{i} .}
\end{aligned}
$$

As the subset $\gamma^{-1}(X)$ is equal to the union of $E_{1}, \ldots, E_{t}$, the subset $\gamma_{\infty}^{-1}\left(S_{\infty, m, p}\right)$ is given by the equality:

$$
\gamma_{\infty}^{-1}\left(S_{\infty, m, p}\right)=\bigcup_{\substack{J \subset\{1, \ldots, t\} \\ J \neq \emptyset}} \bigcup_{\substack{\left(\alpha_{i}, i \in J\right) \in(\mathbb{N} \backslash\{0\} \mid)|J| \\ \sum_{i \in J} a_{i} \alpha_{i} \geq p}} S_{\alpha}^{\infty},
$$

for any $p$. We set:

$$
M:=1+m \sum_{i=1}^{t} a_{i} .
$$

In particular, when $p \geq M, J_{\alpha}$ is not empty as soon as $\sum_{i \in J} a_{i} \alpha_{i} \geq p$. For any $p \geq M$ and any $k \in \mathbb{N}$, we have the equality:

$$
\mathbf{I}_{m, p, k}=\sum_{\substack{J \subset\{1, \ldots, t\} \\ J \neq \emptyset}} \sum_{\substack{\alpha_{i}, i \in J \\ \sum_{i \in J} a_{i} \alpha_{i} \geq p, \alpha_{i} \geq 1}} \mathbf{S}_{J, \alpha, k}
$$

where

$$
\begin{aligned}
& \mathbf{S}_{J, \alpha, k}:=h\left(\left[T_{\alpha}\right]\right)(u v)^{-\left(N-\left|J_{\alpha}\right|\right)(m+1)+\sum_{i \in J \backslash J \alpha} \alpha_{i}} \\
& \quad(u v-1)^{\left|J_{\alpha}\right|}(u v)^{-\left|J_{\alpha}\right|}(u v)^{-\sum_{i \in J} \alpha_{i}\left(b_{i}+1\right)-k \sum_{i \in J \cap\{2, \ldots, t\}} c_{i} \alpha_{i}} .
\end{aligned}
$$

For $J$ nonempty subset in $\{1, \ldots, t\}$, we set:

$$
\mathbf{S}_{J, p, k}^{\prime}=\sum_{\substack{\alpha_{i}, i \in J \\ \sum_{i \in J} a_{i} \alpha_{i} \geq p, \alpha_{i} \geq 1}} \mathbf{S}_{J, \alpha, k}
$$


Thus $\mathbf{I}_{m, p, k}$ is the sum of the $\mathbf{S}_{J, p, k}^{\prime}$. For any $J$, the highest degree terms in $\mathbf{S}_{J, p, k}^{\prime}$ are the terms $\mathbf{S}_{J, \alpha, p, k}$ for which the number

$$
\operatorname{dim} T_{\alpha}-\left(N-\left|J_{\alpha}\right|\right)(m+1)+\sum_{i \in J \backslash J_{\alpha}} \alpha_{i}-\sum_{i \in J} \alpha_{i}\left(b_{i}+1\right)-k \sum_{i \in J \cap\{2, \ldots, t\}} c_{i} \alpha_{i}
$$

is maximal. In particular, for $J=\{1\}$, the highest degree term of $\mathbf{S}_{J, p, k}^{\prime}$ does not depend on $k$.

i) By hypothesis, the image by $\pi_{m, 0}$ of any irreducible component of maximal dimension of $\gamma_{m}^{-1}(S)$ is dense in $E_{1}$. Therefore, for $J \neq\{1\}$, the degree of

$$
h\left(\left[\pi_{m, 0}^{-1}\left(E_{J}^{0}\right) \cap \gamma_{m}^{-1}(S)\right]\right)
$$

is strictly smaller than the degree of the same expression with $J=\{1\}$. As a consequence, when $J \neq\{1\}$, for $\alpha=\left(\alpha_{i}\right)_{i \in J} \in\left(\mathbb{N}^{*}\right)^{|J|}$, the degree of $h\left(\left[T_{\alpha}\right]\right)$ is strictly smaller than the degree of

$$
h\left(\left[\pi_{m, 0}^{-1}\left(E_{\{1\}}^{0}\right) \cap \gamma_{m}^{-1}(S)\right]\right) .
$$

Hence by Lemma 26 and relation (7), when $p$ is big enough, the highest degree term of $\mathbf{I}_{m, p, k}$ is the highest degree term of $\mathbf{S}_{\{1\}, p, k}^{\prime}$. In particular, it does not depend on $k$.

ii) Denote by $\widetilde{S}$ the union of irreducible components of $S$ whose image by $\pi_{m, 0}^{V}$ is dense in $X$. By hypothesis, $\widetilde{S}$ is a nonempty set. We set

$$
\widetilde{S}_{\infty, m, p}:=\left\{\nu \in\left(\pi_{\infty, m}^{V}\right)^{-1}(\widetilde{S}) \mid F_{X}(\nu) \geq p\right\}
$$

and

$$
\widetilde{\mathbf{I}}_{m, p, k}:=h\left(\int_{\widetilde{S}_{\infty, m, p}} \mathbb{L}^{-k F_{Z}(\nu)} \mathrm{d} \mu_{V}(\nu)\right),
$$

for $k>0$. It suffices to prove that the highest degree terms of $\mathbf{I}_{m, p, k}$ and $\widetilde{\mathbf{I}}_{m, p, k}$ are the same, for $p$ big enough. Indeed, if so, we obtain the expected result with $k=0$ in $\mathbf{I}_{m, p, k}$ and $\widetilde{\mathbf{I}}_{m, p, k}$. By (i), the highest degree term of $\widetilde{\mathbf{I}}_{m, p, k}$ does not depend on $k$ for $p$ big enough. In addition, by hypothesis, the highest degree term of $\mathbf{I}_{m, p, k}$ does not depend on $k$ for $p$ big enough. Fix $p$ big enough such that the highest degree terms of both $\widetilde{\mathbf{I}}_{m, p, k}$ and $\mathbf{I}_{m, p, k}$ do not depend on $k$. As $S$ and $\widetilde{S}$ are $\mathbf{K}$-invariant, the function $F_{Z}(\nu)$ is positive for any $\nu$ in $S_{\infty, m, p} \backslash \widetilde{S}_{\infty, m, p}$ since $\mathbf{K}$ has finitely many orbits in $X$. So we get

$$
\lim _{k \rightarrow+\infty} h\left(\int_{S_{\infty, m, p} \backslash \widetilde{S}_{\infty, m, p}} \mathbb{L}^{-k F_{Z}(\nu)} \mathrm{d} \mu_{V}(\nu)\right)=0 .
$$


Hence for $k$ big enough, the highest degree terms of $\mathbf{I}_{m, p, k}$ and $\widetilde{\mathbf{I}}_{m, p, k}$ are the same.

Let $m, p$ be in $\mathbb{N}$ with $p \geq m+1$, and let $S$ be a constructible subset of $J_{m}(V)$. In view of Proposition 28, we wish to investigate the highest degree term of $h \circ \mu_{V}\left(S_{\infty, m, p}\right)$. For $q$ in $\mathbb{N} \cup\{\infty\}$ and $q \geq n$, we denote by $\pi_{q, n}^{X}$ the canonical morphism from $J_{q}(X)$ to $J_{n}(X)$. The following lemma is easy and helpful for Lemma 30.

Lemma 29. - i) If $\nu \in J_{q}(X)$ for $q \in \mathbb{N} \cup\{\infty\}$, then $\pi_{q, n}^{V}(\nu)=\pi_{q, n}^{X}(\nu)$ for any $n \leq q$.

ii) For $\nu \in J_{\infty}(V)$ and $q \in \mathbb{N}^{*}, \pi_{\infty, q-1}^{V}(\nu) \in J_{q-1}(X)$ if and only if $F_{X}(\nu) \geq q$.

We suppose that $S$ satisfies the following conditions:

1) $S$ is contained in $J_{m}(X)$,

2) the image by $\pi_{m, 0}^{V}$ of $S$ is dense in $X$,

3) for any $\nu \in S$, the fiber $\left(\pi_{p-1, m}^{X}\right)^{-1}(\nu)$ is a nonempty set.

Denote by $\widetilde{S}$ the union of irreducible components of $S$ whose image by $\pi_{m, 0}^{V}$ is dense in $X$. By Conditions (1) and (2), $\widetilde{S}$ is a nonempty constructible subset of $J_{m}(X)$. Let $d$ and $\tilde{d}$ be the dimension of $S$ and $\widetilde{S}$ respectively, and let $c$ and $\tilde{c}$ be the number of irreducible components of maximal dimension of $S$ and $\widetilde{S}$ respectively.

Denote by $X_{\text {reg }}$ the smooth part of $X$. Notice that when $S$ is contained in $X_{\text {reg }}$, Condition (3) is automatically satisfied.

Lemma 30. - i) Let $T$ be an irreducible component of $S$ whose image by $\pi_{m, 0}^{V}$ is dense in $X$. Then $\left(\pi_{p-1, m}^{X}\right)^{-1}(T)$ has dimension $\operatorname{dim} T+(N-r)(p-1-m)$.

ii) The highest degree term of $h \circ \mu_{V}\left(\widetilde{S}_{\infty, m, p}\right)$ is $\tilde{c}(u v)^{\tilde{d}-(N-r)(m+1)-p r}$.

iii) The degree of $h \circ \mu_{V}\left(S_{\infty, m, p}\right)$ is at least $d-(N-r)(m+1)-p r$.

iv) Suppose that the highest degree term of $h \circ \mu_{V}\left(S_{\infty, m, p}\right)$ is $\tilde{c}(u v)^{\tilde{d}-(N-r)(m+1)-p r}$. Then $d=\tilde{d}$ and $c=\tilde{c}$.

Proof. - i) By hypothesis, the preimage of $T \cap\left(\pi_{m, 0}^{X}\right)^{-1}\left(X_{\text {reg }}\right)$ by $\pi_{p-1, m}^{X}$ is a locally trivial fibration over $T \cap\left(\pi_{m, 0}^{X}\right)^{-1}\left(X_{\text {reg }}\right)$ with fiber $\mathbb{A}^{(N-r)(p-1-m)}$, whence (i).

ii) Denote by $S^{(p-1)}$ and $\widetilde{S}^{(p-1)}$ the preimage by $\pi_{p-1, m}^{X}$ of $S$ and $\widetilde{S}$ respectively. Using Lemma 29, we can readily check that the relation $\widetilde{S}_{\infty, m, p}=\left(\pi_{\infty, p-1}^{V}\right)^{-1}\left(\widetilde{S}^{(p-1)}\right)$ holds, since $S$ is contained in $J_{m}(X)$. Hence, 
by Proposition 25, (1), we have:

$$
h \circ \mu_{V}\left(\widetilde{S}_{\infty, m, p}\right)=h\left(\left[\widetilde{S}^{(p-1)}\right]\right)(u v)^{-N p} .
$$

By definition of $\widetilde{S}$, the subsets

$$
\left(\pi_{\infty, p-1}^{X}\right)^{-1}\left(\widetilde{S}^{(p-1)}\right) \text { and }\left(\pi_{\infty, m}^{X}\right)^{-1}(\widetilde{S})
$$

are stable sets with respect to $\mu_{X}$ at level $p-1$ and $m$ respectively. Then, by Proposition 25, (1), applied to $\mu_{X}$, we get:

$$
\begin{aligned}
& h \circ \mu_{X}\left(\left(\pi_{\infty, p-1}^{X}\right)^{-1}\left(\widetilde{S}^{(p-1)}\right)\right)=h\left(\left[\widetilde{S}^{(p-1)}\right]\right)(u v)^{-(N-r) p}, \\
& h \circ \mu_{X}\left(\left(\pi_{\infty, m}^{X}\right)^{-1}(\widetilde{S})\right)=h([\widetilde{S}])(u v)^{-(N-r)(m+1)} .
\end{aligned}
$$

As $\left(\pi_{\infty, p-1}^{X}\right)^{-1}\left(\widetilde{S}^{(p-1)}\right)$ and $\left(\pi_{\infty, m}^{X}\right)^{-1}(\widetilde{S})$ coincides, we deduce:

$$
h\left(\left[\widetilde{S}^{(p-1)}\right]\right)=h([\widetilde{S}])(u v)^{(N-r)(p-1-m)} .
$$

But the highest degree term of $h([\widetilde{S}])$ is $\tilde{c}(u v)^{\tilde{d}}$, whence (ii) by Relation (8).

iii) Analogously to (ii), we can write:

$$
h \circ \mu_{V}\left(S_{\infty, m, p}\right)=h\left(\left[S^{(p-1)}\right]\right)(u v)^{-N p} .
$$

Let $T$ be an irreducible component of $S$ of dimension $d$. As $S$ satisfies Condition (3), we have $\pi_{p-1, m}^{X}\left(\left(\pi_{p-1, m}^{X}\right)^{-1}(T)\right)=T$. So there is an irreducible component $T^{\prime}$ of $\left(\pi_{p-1, m}^{X}\right)^{-1}(T)$ whose image by $\pi_{p-1, m}^{X}$ is dense in $T$. As the generic fiber of $\pi_{p-1, m}^{X}: J_{p-1}(X) \longrightarrow J_{m}(X)$ has dimension $(N-r)(p-1-m)$, the dimension of $T^{\prime}$ is at least $d+(N-r)(p-1-m)$. Hence, by Relation (9), the degree of $h \circ \mu_{V}\left(S_{\infty, m, p}\right)$ is at least $d-(N-r)(m+1)-p r$.

iv) By Relation (9), the hypothesis of (iv) means that the highest degree term of $h\left(\left[S^{(p-1)}\right]\right)$ is $\tilde{c}(u v)^{\tilde{d}+(N-r)(p-1-m)}$. In particular, $S^{(p-1)}$ has dimension $\tilde{d}+(N-r)(p-1-m)$ and the number of its irreducible components of maximal dimension is $\tilde{c}$. If $d>\tilde{d}$, then (iii) gives a contradiction. Hence $d=\tilde{d}$. So any irreducible component of maximal dimension of $\widetilde{S}$ is an irreducible component of maximal dimension of $S$. Hence, $c \geq \tilde{c}$. It remains to prove: $c \leq \tilde{c}$. Let $T_{1}, \ldots, T_{q}$ be the irreducible components of $S$. Then

$$
S^{(p-1)}=\left(\pi_{p-1, m}^{X}\right)^{-1}\left(T_{1}\right) \cup \ldots \cup\left(\pi_{p-1, m}^{X}\right)^{-1}\left(T_{q}\right) .
$$

By Condition $(3), \pi_{p-1, m}^{X}\left(\left(\pi_{p-1, m}^{X}\right)^{-1}\left(T_{i}\right)\right)=T_{i}$, for any $i=1, \ldots, q$. Consequently,

$$
\pi_{p-1, m}^{X}\left(T_{i}\right) \varsubsetneqq \pi_{p-1, m}^{X}\left(T_{j}\right)
$$

for any $1 \leq i, j \leq q$, with $i \neq j$. Therefore there is an injection from the set of irreducible components of $S$ into the set of irreducible components of $S^{(p-1)}$. Moreover, by (i), any irreducible component of $S$ whose image by $\pi_{m, 0}^{V}$ is dense in $X$ provides an irreducible component of $S^{(p-1)}$ of maximal 
dimension, since $d=\tilde{d}$. So $\tilde{c} \geq c$, since $\tilde{c}$ is the number of the irreducible components of maximal dimension of $S^{(p-1)}$.

4. Key proposition. - We keep the notations of the previous subsection. We explicitly construct in this subsection a sequence of subsets in $J_{m}(X)$, for $m \geq 1$, to which we apply the results of Subsection 3 . Here, for $q$ in $\mathbb{N} \cup\{\infty\}$ and $q \geq m, \pi_{q, m}$ refers to the canonical projection from $J_{q}(V)$ to $J_{m}(V)$. As $V$ is a vector space, there is a canonical injection from $J_{m}(V)$ into $J_{m+1}(V)$. Furthermore, this injection is a closed immersion. The first projection from $V \times V$ to $V$ is denoted by $\varpi_{1}$. Let $T$ be a closed bicone of $X \times V$ satisfying the two following conditions:

1) $T$ is $\mathbf{K}$-invariant under the diagonal action of $\mathbf{K}$ in $V \times V$,

2) for any $(x, y)$ in $T, y$ is a tangent vector of $X$ at $x$.

We define by induction on $m$ a subset $C_{m}$ of $J_{m}(V)$.

For $m=1$, we denote by $C_{1}$ the image of $T$ by the map $(x, y) \mapsto \nu_{x, y, 1}$.

Let us suppose that the subset $C_{m}$ of $J_{m}(V)$ is defined for some $m \geq 1$. Let $C_{m+1}^{\prime}$ be the image of $C_{m}$ by the canonical injection from $J_{m}(V)$ into $J_{m+1}(V)$. For any $p \geq m+1$, we set

$$
C_{\infty, m+1, p}^{\prime}:=\left\{\nu \in \pi_{\infty, m+1}^{-1}\left(C_{m+1}^{\prime}\right) \mid F_{X}(\nu) \geq p\right\} .
$$

Then we set

$$
C_{m+1}:=\bigcap_{p \geq m+1} \overline{\pi_{\infty, m+1}\left(C_{\infty, m+1, p}^{\prime}\right)},
$$

where $\bar{C}$ denotes the closure of the subset $C$ in $J_{m+1}(V)$. Thus, $C_{m+1}$ is a closed subset of $J_{m+1}(V)$.

For $m \geq 1$, we set

$$
D_{m}:=\left\{(x, y) \in X \times V \mid \nu_{x, y, m} \in C_{m}\right\} .
$$

Lemma 31. - Let $m$ be in $\mathbb{N}^{*}$.

i) If $m \geq 2$, then $C_{m}$ is the closure of $\pi_{\infty, m}\left(C_{\infty, m, p}^{\prime}\right)$ in $J_{m}(V)$, for $p$ big enough.

ii) The subset $C_{m}$ is the image of $D_{m}$ by the map $(x, y) \mapsto \nu_{x, y, m}$. Moreover $C_{m}$ is contained in $J_{m}(X)$.

iii) If $m \geq 2$, then for $p \geq m+1$ and for $\nu \in \pi_{\infty, m}\left(C_{\infty, m, p}^{\prime}\right)$, the fiber at $\nu$ of the canonical morphism from $J_{p-1}(X)$ to $J_{m}(X)$ is a nonempty set.

iv) The set $C_{m}$ is invariant under the action of $\mathbf{K}$ in $J_{m}(V)$.

v) If $\varpi_{1}(T)$ is equal to $X$, then the image by $\pi_{m, 0}$ of $C_{m}$ is equal to $X$. 
Proof. - i) By definition, the sequence

$$
\pi_{\infty, m}\left(C_{\infty, m, m}^{\prime}\right), \pi_{\infty, m}\left(C_{\infty, m, m+1}^{\prime}\right), \ldots
$$

is weakly decreasing. Therefore, by noetherianity, the weakly decreasing sequence

$$
\overline{\pi_{\infty, m}\left(C_{\infty, m, m}^{\prime}\right)}, \overline{\pi_{\infty, m}\left(C_{\infty, m, m+1}^{\prime}\right)}, \ldots
$$

of closed subsets in $J_{m}(V)$ is stationary. Hence for $p$ big enough, $C_{m}$ is the closure of $\pi_{\infty, m}\left(C_{\infty, m, p}^{\prime}\right)$ in $J_{m}(V)$.

ii) It suffices to prove that $C_{m}$ is contained in the image of $D_{m}$ by the map $(x, y) \mapsto \nu_{x, y, m}$ and that $C_{m}$ belongs to $J_{m}(X)$. We prove the statements by induction on $m$. By definition, $C_{1}$ is the image of $T$ by the map $(x, y) \mapsto \nu_{x, y, 1}$ so $C_{1}$ is contained in $J_{1}(X)$ by Condition (2). So $D_{1}$ is equal to $T$, whence the two statements for $m=1$. Let us suppose that $C_{m}$ is the image of $D_{m}$ by the map $(x, y) \mapsto \nu_{x, y, m}$ and let us suppose that $C_{m}$ is contained in $J_{m}(X)$, for some $m \geq 1$. Then $C_{m+1}^{\prime}$ is the image of $D_{m}$ by the map $(x, y) \mapsto \nu_{x, y, m+1}$. By definition, $(x, y)$ belongs to $D_{m+1}$ if and only if $\nu_{x, y, m+1}$ belongs to $C_{m+1}$. As $C_{m+1}$ is contained in $C_{m+1}^{\prime}$, we deduce that $C_{m+1}$ is contained in the image of $D_{m+1}$ by the map $(x, y) \mapsto \nu_{x, y, m+1}$. In addition, for $p \geq m+2$, $\pi_{\infty, m+1}\left(C_{\infty, m+1, p}^{\prime}\right)$ is contained in $J_{m+1}(X)$, by definition of $C_{\infty, m+1, p}^{\prime}$. Hence $C_{m+1}$ is contained in $J_{m+1}(X)$ since $J_{m+1}(X)$ is closed in $J_{m+1}(V)$.

iii) Let $\nu$ be in $\pi_{\infty, m}\left(C_{\infty, m, p}^{\prime}\right)$. Then, there is $\nu^{\prime}$ in $C_{\infty, m, p}^{\prime}$ such that $\pi_{\infty, m}\left(\nu^{\prime}\right)=\nu$. As $\nu^{\prime}$ belongs to $C_{\infty, m, p}^{\prime}$, we have $F_{X}\left(\nu^{\prime}\right) \geq p$. Hence, by Lemma 29], (ii), $\pi_{\infty, p-1}\left(\nu^{\prime}\right)$ belongs to $J_{p-1}(X)$. In addition, since $m \geq 2$, $\pi_{\infty, m}\left(C_{\infty, m, p}^{\prime}\right)$ is contained in $C_{m}$. So by (ii), $\nu$ belongs to $J_{m}(X)$. Therefore, by Lemma 29, (i), $\pi_{\infty, p-1}\left(\nu^{\prime}\right)$ is in the fiber at $\nu$ of the canonical morphism from $J_{p-1}(X)$ to $J_{m}(X)$.

iv) We prove the statement by induction on $m$. As $T$ is $\mathbf{K}$-invariant by Condition (1), $C_{1}$ is $\mathbf{K}$-invariant. We suppose $m \geq 2$ and we suppose that $C_{m}$ is $\mathbf{K}$-invariant. Then $C_{m+1}^{\prime}$ is $\mathbf{K}$-invariant. So $\pi_{\infty, m+1}^{-1}\left(C_{m+1}^{\prime}\right)$ is $\mathbf{K}$-invariant. As $X$ is $\mathbf{K}$-invariant, the function $F_{X}$ is $\mathbf{K}$-invariant. So for any $p \geq m+1$, $C_{\infty, m+1, p}^{\prime}$ is $\mathbf{K}$-invariant. Hence $\pi_{\infty, m+1}\left(C_{\infty, m+1, p}^{\prime}\right)$ and $C_{m+1}$ are $\mathbf{K}$-invariant.

v) We suppose that $\varpi_{1}(T)$ is equal to $X$. Then for any $x$ in $X,(x, 0)$ belongs to $T$ since $T$ is a closed bicone. As a consequence, for any $m \in \mathbb{N}^{*}$, $C_{m}$ contains $\nu_{x, 0, m}$. Hence $\pi_{m, 0}\left(C_{m}\right)$ is equal to $X$.

Proposition 32. - i) For $m$ big enough, we have

$$
D_{m}=\{(x, y) \in T \mid x+t y \in X, \forall t \in \mathbb{C}\} .
$$

ii) We suppose that the image by $\varpi_{1}$ of any irreducible component of maximal dimension of $T$ is equal to $X$. Then, for $m \geq 1$, the image by $\varpi_{1}$ of any irreducible component of maximal dimension of $D_{m}$ is dense in $X$. 
Proof. - i) Let $m$ be in $\mathbb{N}^{*}$ such that $m-1$ is strictly bigger than the degree of any element of a generating family of the ideal of definition of $X$ in the algebra of polynomial functions on $V$. Let $(x, y)$ be in $T$. If $x+t y$ belongs to $X$ for any $t$ in $\mathbb{C}$, then $F_{X}\left(\nu_{x, y}\right)$ is equal to $\infty$. In particular, $C_{m}$ contains $\nu_{x, y, m}$ and $D_{m}$ contains $(x, y)$. Conversely, let us suppose that $D_{m}$ contains $(x, y)$. Then $T$ contains $(x, y)$ since $T$ contains $D_{i}$ for any $i \in \mathbb{N}^{*}$. Moreover, for any $\varphi$ in the ideal of definition of $X$, the function $t \mapsto \varphi(x+t y)$ is divisible by $t^{m}$. So by the choice of $m, \varphi(x+t y)$ is equal to 0 , for any $t \in \mathbb{C}$. In other words, $x+t y$ belongs to $X$ for any $t \in \mathbb{C}$.

ii) By Lemma 31, (ii), the statement is equivalent to the following statement:

for any $m \in \mathbb{N}^{*}$, the image by $\pi_{m, 0}$ of any irreducible component of maximal dimension of $C_{m}$ is dense in $X$.

We prove this statement by induction on $m$. It is true for $m=1$ by hypothesis and by definition of $C_{1}$. Suppose $m \geq 2$ and suppose the statement true for $m-1$. Let $\widetilde{C}_{m}$ be the union of irreducible components of $C_{m}$ whose image by $\pi_{m, 0}$ is dense in $X$. By Lemma 31, (v), $\widetilde{C}_{m}$ is not empty. Let $d$ and $\tilde{d}$ be the dimension of $C_{m}$ and $\widetilde{C}_{m}$ respectively and let $c$ and $\tilde{c}$ be the number of their irreducible components of maximal dimension. It is enough to prove the equalities $d=\tilde{d}$ and $c=\tilde{c}$. In fact, in this case, any irreducible component of maximal dimension of $C_{m}$ is an irreducible component of $\widetilde{C}_{m}$. By Lemma 31. (ii), (iii) and (iv), the conditions of Lemma 30 are satisfied.

For $p \geq m+1$, we set:

$$
\widetilde{C}_{\infty, m, p}:=\left\{\nu \in \pi_{\infty, m}^{-1}\left(\widetilde{C}_{m}\right) \mid F_{X}(\nu) \geq p\right\} .
$$

The image by $\pi_{m, 0}$ of any irreducible component of $\widetilde{C}_{m}$ is dense in $X$. Hence, by Proposition 28, (i), the highest degree term of the element

$$
\widetilde{\mathbf{I}}_{m, p, k}:=h\left(\int_{\widetilde{C}_{\infty, m, p}} \mathbb{L}^{-k F_{Z}(\nu)} \mathrm{d} \mu_{V}(\nu)\right)
$$

of $\mathbb{Z}[u, v]\left[\left[u^{-1}, v^{-1}\right]\right]$ does not depend on $k$, for $p$ big enough. On the other hand, by induction hypothesis, the image by $\pi_{\infty, m-1}$ of any irreducible component of maximal dimension of $C_{m-1}$ is dense in $X$, so the image by $\pi_{\infty, m}$ of any irreducible component of maximal dimension of $C_{m}^{\prime}$ is dense in $X$. Hence by Proposition 28, (i) the highest degree term of the element

$$
\mathbf{I}_{m, p, k}:=h\left(\int_{C_{\infty, m, p}^{\prime}} \mathbb{L}^{-k F_{Z}(\nu)} \mathrm{d} \mu_{V}(\nu)\right)
$$


of $\mathbb{Z}[u, v]\left[\left[u^{-1}, v^{-1}\right]\right]$ does not depend on $k$, for $p$ big enough. At last, applying besides Lemma 31, (i), we may choose $p$ big enough such that the following conditions hold:

1) $C_{m}$ is the closure of $\pi_{\infty, m}\left(C_{\infty, m, p}^{\prime}\right)$,

2) the highest degree terms of $\mathbf{I}_{m, p, k}$ and $\widetilde{\mathbf{I}}_{m, p, k}$ do not depend on $k$.

Using Condition (1), we can easily check that the relation

$$
C_{\infty, m, p}^{\prime}=\left\{\nu \in \pi_{\infty, m}^{-1}\left(\pi_{\infty, m}\left(C_{\infty, m, p}^{\prime}\right)\right) \mid F_{X}(\nu) \geq p\right\}
$$

holds. By Lemma 31, (v), $\pi_{m, 0}\left(C_{m}\right)$ is equal to $X$. So, by Condition (1), the image of $\pi_{\infty, m}\left(C_{\infty, m, p}^{\prime}\right)$ by $\pi_{m, 0}$ is dense in $X$. Then, since Condition (2) holds, it results from Proposition 28, (ii), that the highest degree term of $\mathbf{I}_{m, p, k}$ only depends on the irreducible components of $\pi_{\infty, m}\left(C_{\infty, m, p}^{\prime}\right)$ whose image by $\pi_{m, 0}$ is dense in $X$. In other words, the highest degree terms of $\mathbf{I}_{m, p, k}$ and $\widetilde{\mathbf{I}}_{m, p, k}$ are the same.

By Condition (2) with $k=0$ in $\widetilde{\mathbf{I}}_{m, p, k}$ and Lemma 30, (ii), we deduce that the highest degree term of $\widetilde{\mathbf{I}}_{m, p, k}$ is $\tilde{c}(u v)^{\tilde{d}-(N-r)(m+1)-p r}$. So the highest degree term of $\mathbf{I}_{m, p, k}$ is $\tilde{c}(u v)^{\tilde{d}-(N-r)(m+1)-p r}$, too. Hence, by Condition (2) with $k=0$ in $\mathbf{I}_{m, p, k}$, we deduce that the highest degree term of $h \circ \mu_{V}\left(C_{\infty, m, p}^{\prime}\right)$ is $\tilde{c}(u v)^{\tilde{d}-(N-r)(m+1)-p r}$. Then, by Lemma 30, (iv), the two equalities $d=\tilde{d}$ and $c=\tilde{c}$ hold.

\section{Dimension of the nilpotent bicone via motivic integration}

We prove in this section the main result of this note. As in Section 2, we suppose that $\mathfrak{g}$ is simple and we adopt the notations of previous sections. The aim of this section is the following theorem:

Theorem 33. - i) The principal bicone of $\mathfrak{g}$ is a reduced complete intersection of dimension $3\left(\mathrm{~b}_{\mathfrak{g}}-\mathrm{rkg}+1\right)$.

ii) The subscheme $\mathcal{Y}_{\mathfrak{g}}$ is a reduced complete intersection of dimension $3\left(\mathrm{~b}_{\mathfrak{g}}-\mathrm{rk} \mathfrak{g}\right)+2$. Moreover, any irreducible component of $\mathcal{Y}_{\mathfrak{g}}$ is the intersection of $\mathcal{Y}_{\mathfrak{g}}$ with an irreducible component of $\mathcal{X}_{\mathfrak{g}}$.

iii) The subscheme $\mathcal{Z}_{\mathfrak{g}}$ is a reduced complete intersection of dimension $3\left(b_{\mathfrak{g}}-\mathrm{rk} \mathfrak{g}\right)+1$.

iv) The nilpotent bicone $\mathcal{N}_{\mathfrak{g}}$ is a complete intersection of dimension $3\left(\mathrm{~b}_{\mathfrak{g}}-\mathrm{rkg}\right)$.

Let us give a brief description of our approach to prove Theorem 33 . We plan to apply Corollary 23. Namely, we intend to prove that any irreducible component of maximal dimension of $\mathcal{X}_{\mathfrak{g}}$ satisfies Conditions (1) and (2) of Corollary 23, Then we will deduce Theorem 33, (i), from Lemma 18, (i). Next, 
the statements (ii), (iii) and (iv) of Theorem 33 will be mostly consequences of statement (i). The main point is therefore to study the images by $\varpi_{1}$ and $\varpi_{2}$ of the irreducible components of maximal dimension of $\mathcal{X}_{\mathfrak{g}}, \mathcal{Y}_{\mathfrak{g}}, \mathcal{Z}_{\mathfrak{g}}$ and $\mathcal{N}_{\mathfrak{g}}$. To process, we consider jet schemes of $\mathfrak{X}_{\mathfrak{g}}$ and $\mathfrak{N}_{\mathfrak{g}}$ and we use the results of Section 3 about motivic integration. In fact, we remark that an element $(x, y) \in \mathfrak{g} \times \mathfrak{g}$ belongs to $\mathcal{X}_{\mathfrak{g}}$ or $\mathcal{N}_{\mathfrak{g}}$ if and only if the arc $t \mapsto x+t y$ is an element of $J_{\infty}\left(\mathfrak{X}_{\mathfrak{g}}\right)$ or $J_{\infty}\left(\mathfrak{N}_{\mathfrak{g}}\right)$ respectively. Thus $\mathcal{X}_{\mathfrak{g}}$ and $\mathcal{N}_{\mathfrak{g}}$ can be identified to subsets of $J_{\infty}\left(\mathfrak{X}_{\mathfrak{g}}\right)$ and $J_{\infty}\left(\mathfrak{N}_{\mathfrak{g}}\right)$ respectively. As $\mathfrak{X}_{\mathfrak{g}}$ and $\mathfrak{N}_{\mathfrak{g}}$ are strictly contained in $\mathfrak{g}$, the motivic measure with respect to $\mathfrak{g}$ of the so obtained subsets is zero by Proposition 25, (2). So we cannot expect to obtain any information from these measures. That is why we are going instead to make use of the subtler construction as described in Section 3, 44. We will obtain in this way subsets whose Hodge realization of the motivic measure with respect to $\mathfrak{g}$ provide all the information we need about the varieties $\mathcal{X}_{\mathfrak{g}}$ and $\mathcal{N}_{\mathfrak{g}}$.

1. Two lemmas. - We give in this subsection two lemmas useful for Subsection 2 . We denote by $\theta$ the map

$$
\mathbb{C} \times \mathfrak{g} \times \mathfrak{g} \longrightarrow \mathfrak{g} \times \mathfrak{g},(t, x, y) \mapsto(x, y+t x) .
$$

Lemma 34. - Let $\mathcal{X}$ be a G-invariant irreducible closed bicone of $\mathfrak{g} \times \mathfrak{g}$. Suppose that $\varpi_{1}(\mathcal{X})=\mathfrak{X}_{\mathfrak{g}}$ and that $\theta(\mathbb{C} \times \mathcal{X})$ is contained in $\mathcal{X}$. If $\mathcal{X}_{1}$ and $\mathcal{X}_{2}$ are two irreducible components of the nullvariety of $p_{1,1,1}$ in $\mathcal{X}$ such that $\varpi_{1}\left(\mathcal{X}_{1}\right)=\varpi_{1}\left(\mathcal{X}_{2}\right)=\mathfrak{X}_{\mathfrak{g}}$, then $\mathcal{X}_{1}=\mathcal{X}_{2}$.

Proof. - By hypothesis, we can suppose that $\mathcal{X}_{1}$ and $\mathcal{X}_{2}$ have codimension 1 in $\mathcal{X}$ since $\mathcal{X}$ is irreducible. If $(x, y)$ belongs to the nullvariety of $p_{1,1,1}$ in $\mathcal{X}$, with $x$ semisimple, then $p_{1,1,1}(x, x+y) \neq 0$, since $\langle x, x\rangle \neq 0$ by Lemma 15. (i). By hypothesis, for $i=1,2, \theta\left(\mathbb{C} \times \mathcal{X}_{i}\right)$ is an irreducible constructible subset of $\mathcal{X}$ which strictly contains $\mathcal{X}_{i}$. Hence for $i=1,2, \theta\left(\mathbb{C} \times \mathcal{X}_{i}\right)$ contains a dense open subset of $\mathcal{X}$. As a result, for any $(t, x, y)$ in a certain dense open subset of $\mathbb{C} \times \mathcal{X}_{1}, x$ is semisimple and there exists $\left(t^{\prime}, x^{\prime}, y^{\prime}\right)$ in $\mathbb{C} \times \mathcal{X}_{2}$ such that $\theta\left(t^{\prime}, x^{\prime}, y^{\prime}\right)=\theta(t, x, y)$. From this equality we deduce the equalities:

$$
x^{\prime}=x, y^{\prime}-y=\left(t-t^{\prime}\right) x .
$$

But $\langle x, x\rangle \neq 0$ since $x$ is a semisimple element of $\mathfrak{X}_{\mathfrak{g}}$. On the other hand, $\langle x, y\rangle=\left\langle x, y^{\prime}\right\rangle=0$. So $t^{\prime}=t$ and $(x, y)=\left(x^{\prime}, y^{\prime}\right)$. As a consequence $\mathcal{X}_{1}=\mathcal{X}_{2}$.

Let $\mathrm{T} \mathfrak{X}_{\mathfrak{g}}$ be the total tangent space of $\mathfrak{X}_{\mathfrak{g}}$ and let $\mathrm{T} \mathfrak{N}_{\mathfrak{g}}$ be the total tangent space of $\mathfrak{N}_{\mathfrak{g}}$. They are closed subsets of $\mathfrak{g} \times \mathfrak{g}$. Let $T^{\prime} \mathfrak{X}_{\mathfrak{g}}$ be the nullvariety of $p_{1,1,1}$ in $\mathrm{T} \mathfrak{X}_{\mathfrak{g}}$ and let $\mathrm{T}^{\prime \prime} \mathfrak{X}_{\mathfrak{g}}$ be the nullvariety of $p_{1,0,2}$ in $\mathrm{T}^{\prime} \mathfrak{X}_{\mathfrak{g}}$. 
Lemma 35. - i) The subsets $\mathrm{TX}_{\mathfrak{g}}$ and $\mathrm{T} \mathfrak{N}_{\mathfrak{g}}$ are $G$-invariant closed irreducible bicones, their images by $\varpi_{1}$ are equal to $\mathfrak{X}_{\mathfrak{g}}$ and $\mathfrak{N}_{\mathfrak{g}}$ respectively, and they have dimension $4\left(\mathrm{~b}_{\mathfrak{g}}-\mathrm{rkg}\right)+2$ and $4\left(\mathrm{~b}_{\mathfrak{g}}-\mathrm{rk} \mathfrak{g}\right)$ respectively.

ii) The intersection of $\varpi_{1}^{-1}\left(\mathfrak{N}_{\mathfrak{g}}\right)$ and $\mathrm{T}^{\prime} \mathfrak{X}_{\mathfrak{g}}$ is equal to $\mathrm{T} \mathfrak{N}_{\mathfrak{g}}$.

iii) The subset $\mathrm{T}^{\prime} \mathfrak{X}_{\mathfrak{g}}$ is irreducible of dimension $4\left(\mathrm{~b}_{\mathfrak{g}}-\mathrm{rkg} \mathfrak{g}\right)+1$. Moreover, $\varpi_{1}\left(\mathrm{~T}^{\prime} \mathfrak{X}_{\mathfrak{g}}\right)$ is equal to $\mathfrak{X}_{\mathfrak{g}}$.

iv) The image of any irreducible component of $\mathrm{T}^{\prime \prime} \mathfrak{X}_{\mathfrak{g}}$ by $\varpi_{1}$ is equal to $\mathfrak{X}_{\mathfrak{g}}$.

Proof. - i) As $\mathfrak{X}_{\mathfrak{g}}$ and $\mathfrak{N}_{\mathfrak{g}}$ are $G$-invariant closed cones, $\mathrm{T} \mathfrak{X}_{\mathfrak{g}}$ and $\mathrm{T} \mathfrak{N}_{\mathfrak{g}}$ are $G$-invariant closed bicones. By Theorem 24 and Remark 7 , T $\mathfrak{X}_{\mathfrak{g}}$ is irreducible and has dimension $4\left(\mathrm{~b}_{\mathfrak{g}}-\mathrm{rkg}\right)+2$. Likewise, by Theorem 24 and Remark 7 , $\mathrm{T} \mathfrak{N}_{\mathfrak{g}}$ is irreducible and has dimension $4\left(\mathrm{~b}_{\mathfrak{g}}-\mathrm{rk} \mathfrak{g}\right)$.

ii) For $x$ in G.e and $y$ in $\mathfrak{g},(x, y)$ belongs to $\mathrm{TN}_{\mathfrak{g}}$ if and only if $y$ belongs to $[x, \mathfrak{g}]$. In particular, for any $x$ in G.e and any $y$ in $\mathfrak{g}$ such that $(x, y)$ belongs to $\mathrm{T}_{\mathfrak{g}},(x, y)$ belongs to $\mathrm{T}^{\prime} \mathfrak{X}_{\mathfrak{g}}$ since $x$ is orthogonal to $[x, \mathfrak{g}]$. By (i), the intersection of $\mathrm{T} \mathfrak{N}_{\mathfrak{g}}$ and $\varpi_{1}^{-1}(G . e)$ is dense in $\mathrm{T} \mathfrak{N}_{\mathfrak{g}}$. So $\mathrm{T} \mathfrak{N}_{\mathfrak{g}} \subset \varpi_{1}^{-1}\left(\mathfrak{N}_{\mathfrak{g}}\right) \cap \mathrm{T}^{\prime} \mathfrak{X}_{\mathfrak{g}}$. Let us prove the other inclusion. As $\mathfrak{X}_{\mathfrak{g}}$ is the nullvariety in $\mathfrak{g}$ of $q_{2}, \ldots, q_{\mathrm{rkg}}$, for any $x$ in $\mathfrak{X}_{\mathfrak{g}}$, the subspace of elements $y$ of $\mathfrak{g}$ such that $(x, y)$ belongs to $\mathrm{TX}_{\mathfrak{g}}$ is the intersection of the kernels of the differentials at $x$ of $q_{2}, \ldots, q_{\mathrm{rkg}}$. By definition of the $q_{i}$ (see Subsection 1), for any $x$ in $\mathfrak{N}_{\mathfrak{g}}$, the subset of elements $y$ of $\mathfrak{g}$ such that $(x, y)$ belongs to $\mathrm{TX}_{\mathfrak{g}}$ is the intersection of the kernels of the differentials at $x$ of $p_{2}, \ldots, p_{\mathrm{rkg}}$. Therefore, the intersection of $\mathrm{T}^{\prime} \mathfrak{X}_{\mathfrak{g}}$ and $\varpi_{1}^{-1}\left(\mathfrak{N}_{\mathfrak{g}}\right)$ is contained in $T \mathfrak{N}_{\mathfrak{g}}$, since $\mathfrak{N}_{\mathfrak{g}}$ is the nullvariety of $p_{1}, \ldots, p_{\mathrm{rkg}}$ in $\mathfrak{g}$.

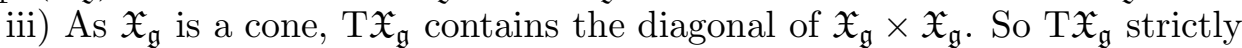
contains $T^{\prime} \mathfrak{X}_{\mathfrak{g}}$. Then by $(\mathrm{i}), \mathrm{T}^{\prime} \mathfrak{X}_{\mathfrak{g}}$ has dimension $4\left(\mathrm{~b}_{\mathfrak{g}}-\mathrm{rk} \mathfrak{g}\right)+1$. Moreover, $\mathrm{T}^{\prime} \mathfrak{X}_{\mathfrak{g}}$ is an equidimensional $G$-invariant closed bicone, since $p_{1,1,1}$ is bihomogeneous and $G$-invariant. Hence by (ii) and Lemma 3 , the image by $\varpi_{1}$ of any irreducible component of $T^{\prime} \mathfrak{X}_{\mathfrak{g}}$ is equal to $\mathfrak{X}_{\mathfrak{g}}$. As $\mathfrak{X}_{\mathfrak{g}}$ is a cone, for any $t$ in $\mathbb{C}$ and any $(x, y)$ in $\mathrm{TX}_{\mathfrak{g}},(x, y+t x)$ belongs to $\mathrm{T} \mathfrak{X}_{\mathfrak{g}}$. Therefore, by Lemma 34 , $\mathrm{T}^{\prime} \mathfrak{X}_{\mathfrak{g}}$ is irreducible.

iv) By (iii), $T^{\prime \prime} \mathfrak{X}_{\mathfrak{g}}$ is equidimensional of dimension at least $4\left(b_{\mathfrak{g}}-r k \mathfrak{g}\right)$. Moreover, since $p_{1,0,2}$ is bihomogeneous and $G$-invariant, $\mathrm{T}^{\prime \prime} \mathfrak{X}_{\mathfrak{g}}$ is a $G$-invariant closed bicone. As $(e, h)$ belongs to $T^{\prime} \mathfrak{X}_{\mathfrak{g}}, T^{\prime \prime} \mathfrak{X}_{\mathfrak{g}}$ is strictly contained in $T^{\prime} \mathfrak{X}_{\mathfrak{g}}$ and $\mathrm{T}^{\prime \prime} \mathfrak{X}_{\mathfrak{g}}$ is equidimensional of dimension $4\left(\mathrm{~b}_{\mathfrak{g}}-\mathrm{rk} \mathfrak{g}\right)$. In addition, as $(e, h)$ belongs to $T \mathfrak{N}_{\mathfrak{g}} \backslash \mathrm{T}^{\prime \prime} \mathfrak{X}_{\mathfrak{g}}$, we deduce from (ii) and Lemma 15, (i), that the intersection of $\mathrm{T}^{\prime \prime} \mathfrak{X}_{\mathfrak{g}}$ and $\varpi_{1}^{-1}\left(\mathfrak{N}_{\mathfrak{g}}\right)$ is equidimensional of dimension $4\left(\mathrm{~b}_{\mathfrak{g}}-\mathrm{rkg} \mathfrak{g}\right)-1$. Hence, by Lemma 3, the image of any irreducible component of $T^{\prime \prime} \mathfrak{X}_{\mathfrak{g}}$ is equal to $\mathfrak{X}_{\mathfrak{g}}$.

2. Proof of Theorem 33. - In this subsection, we apply Proposition 32 to suitable $V, X, T$ and $\mathbf{K}$ in order to prove Theorem 33

Proposition 36. - i) Let $\mathcal{X}$ be an irreducible component of maximal dimension of $\mathcal{X}_{\mathfrak{g}}\left(\right.$ resp. $\left.\mathcal{Y}_{\mathfrak{g}}, \mathcal{Z}_{\mathfrak{g}}\right)$. Then $\varpi_{1}(\mathcal{X})$ is equal to $\mathfrak{X}_{\mathfrak{g}}$. 
ii) Let $\mathcal{X}$ be an irreducible component of maximal dimension of $\mathcal{N}_{\mathfrak{g}}$, then $\varpi_{1}(\mathcal{X})$ is equal to $\mathfrak{N}_{\mathfrak{g}}$.

Proof. - i) Let $\mathbf{K}$ be the subgroup of $\mathrm{GL}(\mathfrak{g})$ generated by $G$ and its homotheties. By Corollary 16, $\mathfrak{X}_{\mathfrak{g}}$ is a $\mathbf{K}$-invariant irreducible closed normal cone and it is a complete intersection in $\mathfrak{g}$ with rational singularities. Moreover, $\mathbf{K}$ has finitely many orbits in $\mathfrak{X}_{\mathfrak{g}}$. Let $T$ be the bicone $T \mathfrak{X}_{\mathfrak{g}}$ (resp. $T^{\prime} \mathfrak{X}_{\mathfrak{g}}, T^{\prime \prime} \mathfrak{X}_{\mathfrak{g}}$ ) of $\mathfrak{X}_{\mathfrak{g}} \times \mathfrak{g}$. By Lemma 35, (i), (resp. (iii), (iv)), the image by $\varpi_{1}$ of any irreducible component of $T$ is equal to $\mathfrak{X}_{\mathfrak{g}}$. Then, by Proposition 32, (i), applied to $V=\mathfrak{g}, X=\mathfrak{X}_{\mathfrak{g}}, T$ and $\mathbf{K}, D_{m}$ is equal to $\mathcal{X}_{\mathfrak{g}}$ (resp. $\mathcal{Y}_{\mathfrak{g}}, \mathcal{Z}_{\mathfrak{g}}$ ), for $m$ big enough. Hence, by Proposition 32, (ii), $\varpi_{1}(\mathcal{X})=\mathfrak{X}_{\mathfrak{g}}$ since $\varpi_{1}(\mathcal{X})$ is closed by Lemma 3 .

ii) Let $\mathbf{K}$ be the subgroup $G$ of $\operatorname{GL}(\mathfrak{g})$. The cone $\mathfrak{N}_{\mathfrak{g}}$ is an irreducible closed normal cone and $\mathbf{K}$ has finitely many orbits in $\mathfrak{N}_{\mathfrak{g}}$. Moreover, it is a complete intersection in $\mathfrak{g}$ and by $\left[\mathbf{H e}\right.$ (Theorem A), $\mathfrak{N}_{\mathfrak{g}}$ has rational singularities. Let $T$ be the bicone $T \mathfrak{N}_{\mathfrak{g}}$ of $\mathfrak{N}_{\mathfrak{g}} \times \mathfrak{g}$. By Lemma 35, (i), $T$ is irreducible and its image by $\varpi_{1}$ is equal to $\mathfrak{N}_{\mathfrak{g}}$. Then, by Proposition 32, (i), applied to $V=\mathfrak{g}, X=\mathfrak{N}_{\mathfrak{g}}$, $T$ and $\mathbf{K}, D_{m}$ is equal to $\mathcal{N}_{\mathfrak{g}}$, for $m$ big enough. Hence, by Proposition 32 , (ii), $\varpi_{1}(\mathcal{X})=\mathfrak{N}_{\mathfrak{g}}$ since $\varpi_{1}(\mathcal{X})$ is closed by Lemma 3 .

Let $d$ be the dimension of $\mathcal{X}_{\mathfrak{g}}$.

Proposition 37. - Let $\mathcal{X}$ be an irreducible component of dimension d of $\mathcal{X}_{\mathfrak{g}}$.

i) The dimension of $\mathcal{Y}_{\mathfrak{g}}$ is $d-1$.

ii) The intersection of $\mathcal{X}$ and $\mathcal{Y}_{\mathfrak{g}}$ is irreducible and has dimension $d-1$. Moreover, this intersection is stable under the involution $(x, y) \mapsto(y, x)$.

iii) The intersection of $\mathcal{X}$ and $\mathcal{Z}_{\mathfrak{g}}$ is an union of irreducible components of $\mathcal{Z}_{\mathfrak{g}}$ of maximal dimension. Moreover, $\mathcal{Z}_{\mathfrak{g}}$ has dimension $d-2$.

iv) The intersection of $\mathcal{N}_{\mathfrak{g}}$ and $\mathcal{X}$ is equidimensional of dimension $d-3$.

v) The dimension of $\mathcal{N}_{\mathfrak{g}}$ is equal to $d-3$.

Proof. - i) Let $\mathcal{X}^{\prime}$ be an irreducible component of $\mathcal{Y}_{\mathfrak{g}}$ of maximal dimension. As $\mathcal{Y}_{\mathfrak{g}}$ is contained in $\mathcal{X}_{\mathfrak{g}}, \mathcal{X}^{\prime}$ is contained in an irreducible component $\mathcal{X}^{\prime \prime}$ of $\mathcal{X}_{\mathfrak{g}}$. Moreover, $\mathcal{X}^{\prime}$ is an irreducible component of the nullvariety of $p_{1,1,1}$ in $\mathcal{X}^{\prime \prime}$. So $\mathcal{X}^{\prime}$ has codimension at most 1 in $\mathcal{X}^{\prime \prime}$. By Proposition 36, (i), $\varpi_{1}\left(\mathcal{X}^{\prime}\right)$ is equal to $\mathfrak{X}_{\mathfrak{g}}$. Hence $\mathcal{X}^{\prime}$ contains $(h, 0)$ since it is a closed bicone. As $\mathcal{X}^{\prime \prime}$ is an irreducible component of $\mathcal{X}_{\mathfrak{g}}$, it is invariant under the action of $\mathrm{GL}_{2}(\mathbb{C})$. Hence $\mathcal{X}^{\prime \prime}$ contains $(h, h)$ and so strictly contains $\mathcal{X}^{\prime}$. So $\mathcal{X}^{\prime}$ has dimension $\operatorname{dim} \mathcal{X}^{\prime \prime}-1$. In particular, $\operatorname{dim} \mathcal{X}^{\prime}$ is at most $d-1$. As any irreducible component of the nullvariety of $p_{1,1,1}$ in $\mathcal{X}$ is contained in $\mathcal{Y}_{\mathfrak{g}}, \mathcal{Y}_{\mathfrak{g}}$ has dimension $d-1$.

ii) As $\mathcal{Y}_{\mathfrak{g}}$ is stable under the involution $(x, y) \mapsto(y, x)$ and $\mathcal{X}$ is stable under the action of $\mathrm{GL}_{2}(\mathbb{C})$, their intersection is stable under the involution $(x, y) \mapsto(y, x)$. By (i), any irreducible component of the intersection of $\mathcal{X}$ and 
$\mathcal{Y}_{\mathfrak{g}}$ has dimension $d-1$. Hence by (i), Proposition 36, (i) and Lemma 34, the intersection of $\mathcal{Y}_{\mathfrak{g}}$ and $\mathcal{X}$ is irreducible since $\theta(\mathbb{C} \times \mathcal{X})$ is contained in $\mathcal{X}$.

iii) Let $\mathcal{Y}$ be the intersection of $\mathcal{Y}_{\mathfrak{g}}$ and $\mathcal{X}$ and let $\mathcal{Z}$ be the intersection of $\mathcal{X}$ and $\mathcal{Z}_{\mathfrak{g}}$. By definition, $\mathcal{Z}$ is the nullvariety of $p_{1,0,2}$ in $\mathcal{Y}$. Moreover, by (ii) and Proposition 36, (i), $\varpi_{2}(\mathcal{Y})$ is equal to $\mathfrak{X}_{\mathfrak{g}}$. Hence by (i) and (ii), any irreducible component of $\mathcal{Z}$ has dimension $d-2$. Let $\mathcal{Z}^{\prime}$ be an irreducible component of $\mathcal{Z}_{\mathfrak{g}}$ of maximal dimension. Then $\operatorname{dim} \mathcal{Z}^{\prime} \geq d-2$. Suppose $\operatorname{dim} \mathcal{Z}^{\prime}>d-2$. As $\mathcal{Z}_{\mathfrak{g}}$ is contained in $\mathcal{Y}_{\mathfrak{g}}, \mathcal{Z}^{\prime}$ is an irreducible component of maximal dimension of $\mathcal{Y}_{\mathfrak{g}}$ by (i). So by Proposition 36 , (i), $\varpi_{2}\left(\mathcal{Z}^{\prime}\right)$ is equal to $\mathfrak{X}_{\mathfrak{g}}$. This is impossible since $\mathcal{Z}^{\prime} \subset \mathcal{Z}_{\mathfrak{g}}$. So $\operatorname{dim} \mathcal{Z}_{\mathfrak{g}}=d-2$ and $\mathcal{Z}$ is an union of irreducible components of dimension $d-2$ of $\mathcal{Z}_{\mathfrak{g}}$.

iv) Let $\mathcal{Y}$ and $\mathcal{Z}$ be as in (iii). As $\mathcal{N}_{\mathfrak{g}}$ is contained in $\mathcal{Z}_{\mathfrak{g}}$, the intersection of $\mathcal{X}$ and $\mathcal{N}_{\mathfrak{g}}$ is contained in $\mathcal{Z}$. By (iii) and Proposition 36, (i), any irreducible component of $\mathcal{Z}$ is not contained in $\mathcal{N}_{\mathfrak{g}}$ and has dimension $d-2$. The intersection of $\mathcal{X}$ and $\mathcal{N}_{\mathfrak{g}}$ is the nullvariety of $p_{1,2,0}$ in $\mathcal{Z}$. Hence this intersection is equidimensional of dimension $d-3$.

v) As $\mathcal{N}_{\mathfrak{g}}$ is contained in $\mathcal{Z}_{\mathfrak{g}}$, any irreducible component of $\mathcal{N}_{\mathfrak{g}}$ is contained in an irreducible component of $\mathcal{Z}_{\mathfrak{g}}$. Moreover, by (iii) and Proposition 36. (i), any irreducible component of dimension $d-2$ of $\mathcal{Z}_{\mathfrak{g}}$ is not contained in $\mathcal{N}_{\mathfrak{g}}$. Hence the dimension of $\mathcal{N}_{\mathfrak{g}}$ is at most $d-3$. So, by (iv), $\mathcal{N}_{\mathfrak{g}}$ has dimension $d-3$.

Corollary 38. - i) The subscheme $\mathcal{X}_{\mathfrak{g}}$ is equidimensional of dimension $3\left(\mathrm{~b}_{\mathfrak{g}}-\mathrm{rkg}+1\right)$. Moreover, any irreducible component of $\mathcal{X}_{\mathfrak{g}}$ has a nonempty intersection with the intersection of $\Omega_{\mathfrak{g}}$ and $\mathcal{Z}_{\mathfrak{g}}$.

ii) The subscheme $\mathcal{Y}_{\mathfrak{g}}$ is equidimensional of dimension $3\left(\mathrm{~b}_{\mathfrak{g}}-\mathrm{rkg}\right)+2$. Moreover, any irreducible component of $\mathcal{Y}_{\mathfrak{g}}$ has a nonempty intersection with $\Omega_{\mathfrak{g}}$.

iii) The subscheme $\mathcal{Z}_{\mathfrak{g}}$ is equidimensional of dimension $3\left(\mathrm{~b}_{\mathfrak{g}}-\mathrm{rkg}\right)+1$. Moreover, any irreducible component of $\mathcal{Z}_{\mathfrak{g}}$ has a nonempty intersection with $\Omega_{\mathfrak{g}}$.

Proof. - i) Let $\mathcal{X}$ be an irreducible component of dimension $d$ of $\mathcal{X}_{\mathfrak{g}}$. Let $\mathcal{Z}$ and $\mathcal{N}$ be the intersections of $\mathcal{X}$ with $\mathcal{Z}_{\mathfrak{g}}$ and $\mathcal{N}_{\mathfrak{g}}$ respectively. By Proposition 37. (iii) and (iv), $\mathcal{Z}$ and $\mathcal{N}$ are equidimensional of dimension $d-2$ and $d-3$ respectively. Hence by Proposition [37, (v), $\mathcal{N}$ is an union of irreducible components of maximal dimension of $\mathcal{N}_{\mathfrak{g}}$. As any irreducible component of $\mathcal{N}_{\mathfrak{g}}$ is stable under the action of $\mathrm{GL}_{2}(\mathbb{C})$, the image by $\varpi_{2}$ of any irreducible component of $\mathcal{N}$ is equal to $\mathfrak{N}_{\mathfrak{g}}$ by Proposition [36, (ii). Moreover, each irreducible component of $\mathcal{Z}$ contains an irreducible component of $\mathcal{N}$ since $\mathcal{N}$ is the nullvariety of $p_{1,2,0}$ in $\mathcal{Z}$. Hence the image by $\varpi_{2}$ of any irreducible component of $\mathcal{Z}$ is equal to $\mathfrak{N}_{\mathfrak{g}}$. By Proposition 37, (iii), each irreducible component of $\mathcal{Z}$ is an irreducible component of $\mathcal{Z}_{\mathfrak{g}}$ of maximal dimension. Hence by Proposition 
36. (i), the image by $\varpi_{1}$ of any irreducible component of $\mathcal{Z}$ is equal to $\mathfrak{X}_{\mathfrak{g}}$. So $\mathcal{Z}$ satisfies Conditions (1) and (2) of Corollary 23, Hence by Corollary 23, $\mathcal{Z}$ has a nonempty intersection with $\Omega_{\mathfrak{g}}$ and $\mathcal{X}$ has dimension $3\left(\mathrm{~b}_{\mathfrak{g}}-\mathrm{rk} \mathfrak{g}+1\right)$. But by Lemma 18, (i), any irreducible component of $\mathcal{X}_{\mathfrak{g}}$ has dimension at least $3\left(b_{\mathfrak{g}}-\mathrm{rkg}+1\right)$. Hence $\mathcal{X}_{\mathfrak{g}}$ is equidimensional of dimension $3\left(\mathrm{~b}_{\mathfrak{g}}-\mathrm{rk} \mathfrak{g}+1\right)$.

ii) By Proposition 37, (ii) and Lemma 19, (ii), $\mathcal{Y}_{\mathfrak{g}}$ is equidimensional of dimension $3\left(b_{\mathfrak{g}}-\mathrm{rkg}\right)+2$. Moreover, any irreducible component of $\mathcal{Y}_{\mathfrak{g}}$ is the intersection of $\mathcal{Y}_{\mathfrak{g}}$ and an irreducible component of $\mathcal{X}_{\mathfrak{g}}$. Hence by (i) and Corollary 23, any irreducible component of $\mathcal{Y}_{\mathfrak{g}}$ has a nonempty intersection with $\Omega_{\mathfrak{g}}$.

iii) By Proposition 37, (ii) and Lemma 19, $\mathcal{Z}_{\mathfrak{g}}$ is equidimensional of dimension $3\left(\mathrm{~b}_{\mathfrak{g}}-\mathrm{rkg}\right)+1$. Let $\mathcal{Z}$ be an irreducible component of $\mathcal{Z}_{\mathfrak{g}}$. By Proposition 36. (i), $\varpi_{1}(\mathcal{Z})$ is equal to $\mathfrak{X}_{\mathfrak{g}}$. Hence by (i) and Proposition [37, (v), the nullvariety of $p_{1,2,0}$ in $\mathcal{Z}$ is an union of irreducible components of $\mathcal{N}_{\mathfrak{g}}$ of maximal dimension. Then by Proposition $\left[36\right.$, (ii), $\varpi_{2}(\mathcal{Z})$ is equal to $\mathfrak{N}_{\mathfrak{g}}$ since any irreducible component of $\mathcal{N}_{\mathfrak{g}}$ is stable under the involution $(x, y) \mapsto(y, x)$. So by Lemma 22, $\mathcal{Z}$ has a nonempty intersection with $\Omega_{\mathfrak{g}}$.

We can now give the proof of Theorem 33;

Proof. - i) By Corollary [38, (i), Lemma 18, (i) and Lemma 20, (ii), $\mathcal{X}_{\mathfrak{g}}$ is a complete intersection of dimension $3\left(\mathrm{~b}_{\mathfrak{g}}-\mathrm{rkg}+1\right)$ and any irreducible component of $\mathcal{X}_{\mathfrak{g}}$ contains a smooth point. So $\mathcal{X}_{\mathfrak{g}}$ is generically reduced and by Ma $($ Ch. $8, \S 23)$, the scheme $\mathcal{X}_{\mathfrak{g}}$ is reduced.

ii) By Corollary 38, (ii), Lemma 19, (ii) and Lemma 20, (ii), $\mathcal{Y}_{\mathfrak{g}}$ is a complete intersection of dimension $3\left(b_{\mathfrak{g}}-r k \mathfrak{g}\right)+2$ and any irreducible component of $\mathcal{Y}_{\mathfrak{g}}$ contains a smooth point. So $\mathcal{Y}_{\mathfrak{g}}$ is generically reduced and by $[\mathbf{M a}](\mathrm{Ch} .8, \S 23)$, the scheme $\mathcal{Y}_{\mathfrak{g}}$ is reduced. Moreover, by Proposition 37, (ii), any irreducible component of $\mathcal{Y}_{\mathfrak{g}}$ is the intersection of $\mathcal{Y}_{\mathfrak{g}}$ and an irreducible component of $\mathcal{X}_{\mathfrak{g}}$.

iii) By Corollary 38, (iii), Lemma 18, (i) and Lemma 20, (ii), $\mathcal{Z}_{\mathfrak{g}}$ is a complete intersection of dimension $3\left(\mathrm{~b}_{\mathfrak{g}}-\mathrm{rk} \mathfrak{g}\right)+1$ and any irreducible component of $\mathcal{Z}_{\mathfrak{g}}$ contains a smooth point. So $\mathcal{Z}_{\mathfrak{g}}$ is generically reduced and by $\mathbf{M a}(\mathrm{Ch}$. 8, $\S 23)$, the scheme $\mathcal{Z}_{\mathfrak{g}}$ is reduced.

iv) As $\mathcal{N}_{\mathfrak{g}}$ is the nullvariety of $b_{\mathfrak{g}}+$ rk $\mathfrak{g}$ polynomial functions, any irreducible component of $\mathcal{N}_{\mathfrak{g}}$ has dimension at least $3\left(\mathrm{~b}_{\mathfrak{g}}-\mathrm{rk} \mathfrak{g}\right)$. Hence by Corollary 38. (i) and Proposition 37, (v), $\mathcal{N}_{\mathfrak{g}}$ is a complete intersection of dimension $3\left(\mathrm{~b}_{\mathfrak{g}}-\mathrm{rk} \mathfrak{g}\right)$.

We deduce Theorem 2 from Theorem 33, (i) and Proposition 36, (i) and we deduce the main part of Theorem 1 from Theorem 33, (iv) and Proposition 36. (ii). To complete the proof of Theorem 1, it only remains to show that $\mathcal{N}_{\mathfrak{g}}$ is a nonreduced scheme, what we will do in Section 6 . 


\section{Applications to invariant theory}

In this section, we present various applications of previous results to invariant theory. In this section, $\mathfrak{g}$ is supposed to be simple again.

1. Properties of the algebra of $\operatorname{pol}_{2} \mathrm{~S}(\mathfrak{g})^{\mathfrak{g}}$. - We denote by $\operatorname{pol}_{2} \mathrm{~S}(\mathfrak{g})^{\mathfrak{g}}$ the subalgebra of $S(\mathfrak{g}) \otimes_{\mathbb{C}} \mathrm{S}(\mathfrak{g})$ generated by the 2-order polarizations of elements of $\mathbf{S}(\mathfrak{g})^{\mathfrak{g}}$. Since the polynomials $p_{1}, \ldots, p_{\text {rkg }}$ generate $\mathrm{S}(\mathfrak{g})^{\mathfrak{g}}$, the polynomials $p_{i, m, n}$, where $i=1, \ldots, \mathrm{rkg}, m+n=d_{i}$ generate $\operatorname{pol}_{2} \mathrm{~S}(\mathfrak{g})^{\mathfrak{g}}$ as graded algebra. Besides, the morphism $\sigma$ introduced in Section 1, \&4, is the morphism of affine varieties whose comorphism is the canonical injection from $\operatorname{pol}_{2} \mathrm{~S}(\mathfrak{g})^{\mathfrak{g}}$ into $S(\mathfrak{g}) \otimes_{\mathbb{C}} \mathrm{S}(\mathfrak{g})$. Then, by Proposition 13, we can state:

Proposition 39. - The subalgebra $\operatorname{pol}_{2} \mathrm{~S}(\mathfrak{g})^{\mathfrak{g}}$ is a polynomial algebra in $\mathrm{b}_{\mathfrak{g}}+\mathrm{rkg}$ variables.

We deduce from Proposition 39:

Theorem 40. - The morphism $\sigma$ is faithfully flat. Equivalently, the extension $\mathrm{S}(\mathfrak{g}) \otimes_{\mathbb{C}} \mathrm{S}(\mathfrak{g})$ of $\operatorname{pol}_{2} \mathrm{~S}(\mathfrak{g})^{\mathfrak{g}}$ is faithfully flat.

Proof. - As $\operatorname{pol}_{2} \mathrm{~S}(\mathfrak{g})^{\mathfrak{g}}$ is generated by homogeneous functions, the fiber at 0 of the morphism $\sigma$ has maximal dimension. On the other hand, by Proposition 39, $\operatorname{pol}_{2} \mathrm{~S}(\mathfrak{g})^{\mathfrak{g}}$ is a polynomial algebra in $\mathrm{b}_{\mathfrak{g}}+\mathrm{rk} \mathfrak{g}$ variables. So $\sigma$ is an equidimensional morphism and by $\mathbf{M a}$ (Ch. 8, Theorem 21.3), $\sigma$ is a flat morphism. In particular by $\mathbf{H a}$ (Ch. III, Exercise 9.4), it is an open morphism whose image contains 0 . So $\sigma$ is surjective. Hence $\sigma$ is faithfully flat, according to Ma $($ Ch. 3, Theorem 7.2).

2. The nullcone. - The nullcone $\mathcal{V}_{\mathfrak{g}}$ of the $G$-module $\mathfrak{g} \times \mathfrak{g}$ is the nullvariety in $\mathfrak{g} \times \mathfrak{g}$ of the augmentation ideal of the ideal of $S(\mathfrak{g}) \otimes_{\mathbb{C}} \mathrm{S}(\mathfrak{g})$ generated by $\left(S(\mathfrak{g}) \otimes_{\mathbb{C}} S(\mathfrak{g})\right)^{\mathfrak{g}}$. The nullcone plays a leading part in invariant theory. It is studied in [Ri5], Po, [LMP, and recently in [KrW1 and [KrW2 . By KrW1, $\mathcal{V}_{\mathfrak{g}}$ is irreducible and has dimension $3\left(\mathrm{~b}_{\mathfrak{g}}-\mathrm{rkg}\right)$. Furthermore, N. Wallach and $\mathrm{H}$. Kraft conjecture in $\mathbf{K r W 2}$ that $\mathcal{V}_{\mathfrak{g}}$ is an irreducible component of $\mathcal{N}_{\mathfrak{g}}$. Clearly, Theorem 1 confirms that conjecture. Thereby, we claim:

Theorem 41. - The nullcone $\mathcal{V}_{\mathfrak{g}}$ is an irreducible component of the nilpotent bicone $\mathcal{N}_{\mathfrak{g}}$.

Let $k$ be in $\mathbb{N}^{*}$ and let us denote by $\mathfrak{g}^{k}$ the $k$-th cartesian power of $\mathfrak{g}$. We extend the previous notions to $\mathfrak{g}^{k}$. Let $\mathrm{S}\left(\mathfrak{g}^{k}\right)$ be the symmetric algebra of $\mathfrak{g}^{k}$ and let $\mathrm{S}\left(\mathfrak{g}^{k}\right)^{\mathfrak{g}}$ be the subalgebra of its invariant elements under the diagonal action of $G$ in $\mathfrak{g}^{k}$. Then we denote by $\mathcal{V}_{\mathfrak{g}}^{(k)}$ the nullvariety in $\mathfrak{g}^{k}$ of the 
augmentation ideal $\mathrm{S}\left(\mathfrak{g}^{k}\right)_{+}^{\mathfrak{g}}$ of $\mathrm{S}\left(\mathfrak{g}^{k}\right)^{\mathfrak{g}}$. For $\varphi$ in $\mathrm{S}(\mathfrak{g})$, the $k$-order polarizations $\varphi_{i_{1}, \ldots, i_{k}}$ of $\varphi$ are defined by the relation:

$$
\varphi\left(t_{1} x_{1}+\cdots+t_{k} x_{k}\right)=\sum_{\left(i_{1}, \ldots, i_{k}\right) \in \mathbb{N}^{k}} t_{1}^{i_{1}} \ldots t_{k}^{i_{k}} \varphi_{i_{1}, \ldots, i_{k}}\left(x_{1}, \ldots, x_{k}\right),
$$

for $\left(x_{1}, \ldots, x_{k}\right)$ in $\mathfrak{g}^{k}$ and $\left(t_{1}, \ldots, t_{k}\right)$ in $\mathbb{C}^{k}$. Then, we denote by $\operatorname{pol}_{k} \mathrm{~S}(\mathfrak{g})^{\mathfrak{g}}$ the subalgebra of $\mathrm{S}\left(\mathfrak{g}^{k}\right)$ generated by the $k$-order polarizations of elements in $\mathrm{S}(\mathfrak{g})^{\mathfrak{g}}$. Then $\operatorname{pol}_{k} \mathrm{~S}(\mathfrak{g})^{\mathfrak{g}}$ is an homogeneous subalgebra of $\mathrm{S}\left(\mathfrak{g}^{k}\right)$ and its augmentation ideal $\operatorname{pol}_{k} \mathrm{~S}(\mathfrak{g})_{+}^{\mathfrak{g}}$ is generated by the $k$-order polarizations of the elements of $\mathrm{S}(\mathfrak{g})_{+}^{\mathfrak{g}}$. We denote by $\mathcal{N}_{\mathfrak{g}}^{(k)}$ the nullvariety in $\mathfrak{g}^{k}$ of the ideal $\operatorname{pol}_{k} \mathrm{~S}(\mathfrak{g})_{+}^{\mathfrak{g}}$. Obviously, $\mathcal{V}_{\mathfrak{g}}^{(1)}=\mathcal{N}_{\mathfrak{g}}^{(1)}=\mathfrak{N}_{\mathfrak{g}}, \mathcal{V}_{\mathfrak{g}}^{(2)}=\mathcal{V}_{\mathfrak{g}}$ and $\mathcal{N}_{\mathfrak{g}}^{(2)}=\mathcal{N}_{\mathfrak{g}}$.

The index of polarization of $\mathfrak{g}$, denoted by polind $(\mathfrak{g})$, is defined in LMP as being the upper bound of $k \in \mathbb{N}^{*}$ such that $\mathcal{V}_{\mathfrak{g}}^{(k)}=\mathcal{N}_{\mathfrak{g}}^{(k)}$. The inequality $\operatorname{polind}(\mathfrak{g}) \geq 1$ obviously always holds. We wish next to establish an equality.

Recall that $(e, h, f)$ is a principal $\mathfrak{s l}_{2}$-triple of $\mathfrak{g}$.

Lemma 42. - Let $x$ be in $\mathfrak{g}$ satisfying $(\operatorname{ad} x)^{2}(e)=0$. Then $(e,[x, e])$ belongs to $\mathcal{N}_{\mathfrak{g}}$.

Proof. - Since $(\operatorname{ad} x)^{2}(e)=0$, we have $\exp (\operatorname{tad} x)(e)=e+t[x, e]$, for any $t$ in $\mathbb{C}$. As $\mathfrak{N}_{\mathfrak{g}}$ is a closed cone in $\mathfrak{g}$, the element $s e+t[x, e]$ belongs to $\mathfrak{N}_{\mathfrak{g}}$, for any $(s, t)$ in $\mathbb{C}^{2}$. So $(e,[x, e])$ belongs to $\mathcal{N}_{\mathfrak{g}}$.

By the Hilbert-Mumford criterion $[\mathbf{K r}$ (Kap. II), an element $(x, y)$ of $\mathfrak{g} \times \mathfrak{g}$ belongs to the nullcone $\mathcal{V}_{\mathfrak{g}}$ if and only if there is a one-parameter subgroup $\lambda: \mathbb{C}^{*} \rightarrow G$ such that $\lim _{t \rightarrow 0} \lambda(t)(x, y)=0$. In particular, if $(x, y)$ belongs to the nullcone, then the subalgebra $L$ generated by $x$ and $y$ is contained in the nilpotent cone. Hence $L$ is nilpotent and so contained in a Borel subalgebra of $\mathfrak{g}$. Conversely, if $x$ and $y$ belong to the nilpotent radical of a common Borel subalgebra, then the previous criterion applies to $(x, y)$. To summarize, we have actually shown:

Lemma 43. - The element $(x, y)$ of $\mathfrak{g} \times \mathfrak{g}$ belongs to the nullcone $\mathcal{V}_{\mathfrak{g}}$ if and only if $x$ and $y$ belong to the nilpotent radical of a common Borel subalgebra.

The following proposition has already been noticed in [LMP](Theorem 3.16). We provide here a shorter proof.

Proposition 44. - We have:

$$
\operatorname{polind}(\mathfrak{g})= \begin{cases}\infty, & \text { if } \mathfrak{g} \text { is isomorphic to } \mathfrak{s l}_{2}(\mathbb{C}) \\ 1, & \text { otherwise. }\end{cases}
$$


Proof. - Suppose first $\operatorname{dim} \mathfrak{g}=3$. The elements $x$ in $\mathfrak{g}$ such that the subspace generated by $x$ and $e$ is contained in the nilpotent cone of $\mathfrak{g}$ are colinear to $e$. Hence $\mathcal{V}_{\mathfrak{g}}^{(2)}=\mathcal{N}_{\mathfrak{g}}$, and $\mathcal{N}_{\mathfrak{g}}$ is irreducible. Then for $k \geq 2$ and $x_{1}, \ldots, x_{k}$ in $\mathfrak{g}$, the subspace generated by $x_{1}, \ldots, x_{k}$ is contained in $\mathfrak{N}_{\mathfrak{g}}$ if and only if its dimension is smaller than 1 . So $\mathcal{V}_{\mathfrak{g}}^{(k)}$ is equal to $\mathcal{N}_{\mathfrak{g}}^{(k)}$ for any $k$.

Suppose now $\operatorname{dim} \mathfrak{g}>3$. As $d_{1}, \ldots, d_{\mathrm{rkg}}$ is a weakly increasing sequence, $-2\left(d_{\mathrm{rkg}}-1\right)$ is the smallest eigenvalue of ad $h$. Let $v$ be a non zero eigenvector of $\operatorname{ad} h$ of eigenvalue $-2\left(d_{\mathrm{rkg}}-1\right)$ and set $v_{0}=[v, e]$. Then $(\operatorname{ad} v)^{2}(e)$ is an eigenvector of ad $h$ of eigenvalue $-4\left(d_{\mathrm{rkg}}-1\right)+2$. As $\mathfrak{g}$ is simple and $\operatorname{dim} \mathfrak{g}>3$, we have $d_{\mathrm{rkg}}>2$ and $-4\left(d_{\mathrm{rkg}}-1\right)+2<-2\left(d_{\mathrm{rkg}}-1\right)$. So $(\operatorname{ad} v)^{2}(e)$ is equal to 0 . Hence, by Lemma 42, $\left(e, v_{0}\right)$ belongs to $\mathcal{N}_{\mathfrak{g}}$. As $v_{0}$ is an eigenvector of ad $h$ of negative eigenvalue, $\mathfrak{b}$ does not contain $v_{0}$. Since $\mathfrak{b}$ is the unique Borel subalgebra which contains $e$, we deduce from Lemma 43 that $\left(e, v_{0}\right)$ does not belong to $\mathcal{V}_{\mathfrak{g}}$. So $\mathcal{V}_{\mathfrak{g}}$ is strictly contained in $\mathcal{N}_{\mathfrak{g}}$. As a result, for $k \geq 2$, $\mathcal{V}_{\mathfrak{g}}^{(k)}$ is strictly contained in $\mathcal{N}_{\mathfrak{g}}^{(k)}$ since $\mathcal{V}_{\mathfrak{g}} \times\left\{0_{\mathfrak{g}^{k-2}}\right\}$ and $\mathcal{N}_{\mathfrak{g}} \times\left\{0_{\mathfrak{g}^{k-2}}\right\}$ are the intersections of $\mathcal{V}_{\mathfrak{g}}^{(k)}$ and $\mathcal{N}_{\mathfrak{g}}^{(k)}$ with $\mathfrak{g} \times \mathfrak{g} \times\left\{0_{\mathfrak{g}^{k-2}}\right\}$ respectively.

Remark 8. - The nullcone has a natural structure of scheme. This scheme is irreducible. Furthermore, for $\operatorname{dim} \mathfrak{g}>3$, it is not reduced. If this scheme were reduced, the extension $S(\mathfrak{g}) \otimes_{\mathbb{C}} \mathrm{S}(\mathfrak{g})$ of $\left(\mathrm{S}(\mathfrak{g}) \otimes_{\mathbb{C}} \mathrm{S}(\mathfrak{g})\right)^{\mathfrak{g}}$ would be flat by arguments used in Kos2. But this extension is not equidimensional since the $G$-orbit in $\mathfrak{g} \times \mathfrak{g}$ in general position has dimension $2 \mathrm{~b}_{\mathfrak{g}}-\mathrm{rkg}$ and $2 \mathrm{~b}_{\mathfrak{g}}-\mathrm{rk} \mathfrak{g}>$ $3\left(\mathrm{~b}_{\mathfrak{g}}-\mathrm{rkg}\right)$.

\section{Additional properties of the nilpotent bicone}

In this section, we give additional results about the nilpotent bicone $\mathcal{N}_{\mathfrak{g}}$. We assert in the first subsection that $\mathcal{N}_{\mathfrak{g}}$ is not reduced. Next, we discuss in the second subsection the number of its irreducible components. As before, we fix a principal $\mathfrak{s l}_{2}$-triple $(e, h, f)$ in $\mathfrak{g}$ and we use the notations of the Introduction, (33.

1. Nonreducibility of $\mathcal{N}_{\mathfrak{g}}$ - - According to $\mathbf{M a}(\S 23)$, a Noetherian ring $A$ is reduced if and only if $A$ satisfies Conditions $\left(R_{0}\right)$ and $\left(S_{1}\right)$. By Theorem 33. the subscheme $\mathcal{N}_{\mathfrak{g}}$ of $\mathfrak{g} \times \mathfrak{g}$ defined by the ideal $\operatorname{pol}_{2} \mathrm{~S}(\mathfrak{g})_{+}^{\mathfrak{g}}$ is a complete intersection in $\mathfrak{g} \times \mathfrak{g}$. Therefore the quotient ring $\left(\mathrm{S}(\mathfrak{g}) \otimes_{\mathbb{C}} \mathrm{S}(\mathfrak{g})\right) / \operatorname{pol}_{2} \mathrm{~S}(\mathfrak{g})_{+}^{\mathfrak{g}}$ is Cohen-Macaulay, according to $\mathbf{M a}$ (Ch. 8, Theorem 21.3). It is natural to ask if $\left(\mathrm{S}(\mathfrak{g}) \otimes_{\mathbb{C}} \mathrm{S}(\mathfrak{g})\right) / \mathrm{pol}_{2} \mathrm{~S}(\mathfrak{g})_{+}^{\mathfrak{g}}$ is reduced. The following theorem answers this question negatively.

Theorem 45. - The scheme $\mathcal{N}_{\mathfrak{g}}$ is not reduced. 
Proof. - In view of the preceding remarks, it suffices to find an irreducible component of $\mathcal{N}_{\mathfrak{g}}$ which does not contain any smooth point of $\mathcal{N}_{\mathfrak{g}}$. Hence, according to Proposition [13, Lemma 20, (ii) and Theorem 41, it is enough to prove that the nullcone $\mathcal{V}_{\mathfrak{g}}$ has an empty intersection with the open subset $\Omega_{\mathfrak{g}}$. We suppose that this intersection is not empty and we expect a contradiction. Let $(x, y)$ be in the intersection of $\mathcal{V}_{\mathfrak{g}}$ and $\Omega_{\mathfrak{g}}$. By Lemma 43, we can suppose that $x$ and $y$ belongs to $\mathfrak{u}$. By Lemma 7, (ii), for any $(a, b)$ in $\mathbb{C}^{2} \backslash\{(0,0)\}$, the centralizer of $a x+b y$ is contained in $\mathfrak{u}$. Therefore, it results from Lemma 4 that the subspace $\mathfrak{V}^{\prime}(x, y)$ is contained in $\mathfrak{u}$. Moreover, by Lemma 8 , (iii), $\mathfrak{V}^{\prime}(x, y)$ is equal to $\mathfrak{V}(x, y)$. But by Lemma 8 , (i), $\mathfrak{V}(x, y)$ has dimension $\mathrm{b}_{\mathfrak{g}}>\operatorname{dim} \mathfrak{u}$, since $(x, y)$ belongs to $\Omega_{\mathfrak{g}}$, whence the expected contradiction.

The proof of Theorem 1 is now completed by Theorem 45 .

\section{Irreducible components of $\mathcal{N}_{\mathfrak{g}}$. - Denote by $\Phi$ the map}

$$
G \times \mathfrak{g} \times \mathfrak{g} \longrightarrow \mathfrak{g} \times \mathfrak{g},(g, x, y) \mapsto(g(x), g(y)) .
$$

For a subset $X$ of $\mathfrak{g} \times \mathfrak{g}$, the closure in $\mathfrak{g} \times \mathfrak{g}$ of the image of $G \times X$ by $\Phi$ is denoted by $X^{\Phi}$. For $Y$ subset of $\mathfrak{g} \times \mathfrak{g}$ and $x$ in $\mathfrak{g}$, we denote by $Y_{x}$ the subset of elements $y$ in $\mathfrak{g}$ such that $(x, y)$ belongs to $Y$.

Lemma 46. - Let $Y$ be a $G$-invariant closed bicone of $\mathfrak{g} \times \mathfrak{g}$ and let $x_{0}$ be a nilpotent element of $\mathfrak{g}$. Suppose that the image of any irreducible component of $Y$ by $\varpi_{1}$ is equal to the closure in $\mathfrak{g}$ of G.x. $x_{0}$. Suppose besides that there exists a subvariety $T$ of $G$ such that the map $g \mapsto g\left(x_{0}\right)$ is an isomorphism from $T$ to an open subset of $G . x_{0}$ containing $x_{0}$.

i) The subset $Y$ is equal to $\left(\left\{x_{0}\right\} \times Y_{x_{0}}\right)^{\Phi}$.

ii) If $Z$ is an irreducible component of $Y$, then $Z_{x_{0}}$ is irreducible.

iii) The map $X \mapsto\left(\left\{x_{0}\right\} \times X\right)^{\Phi}$ is a bijection from the set of irreducible components of $Y_{x_{0}}$ to the set of irreducible components of $Y$.

Proof. - i) By our hypothesis, for any irreducible component $Z$ of $Y, \varpi_{1}(Z)$ is the closure of G.x. So G. $x_{0}$ is a dense open subset in $\varpi_{1}(Z)$. Then the subset $Y^{\prime}$ of elements of $Y$ whose first component is in $G . x_{0}$ is a dense open subset in $Y$. As $Y$ is a $G$-invariant bicone, $Y_{x_{0}}$ is a cone and $Y^{\prime}$ is the image by $\Phi$ of $G \times\left\{x_{0}\right\} \times Y_{x_{0}}$. So $Y$ is equal to $\left(\left\{x_{0}\right\} \times Y_{x_{0}}\right)^{\Phi}$.

ii) Let $Z$ be an irreducible component of $Y$. Then $Z$ is a $G$-invariant closed bicone. We denote by $\Omega$ the image of $T$ by the map $g \mapsto g\left(x_{0}\right)$ and we denote by $\widetilde{Z}$ the subset of elements $(x, y)$ in $Z$ such that $x$ belongs to $\Omega$. By our hypothesis, $\widetilde{Z}$ is a nonempty open subset of $Z$. In particular, it is irreducible. Let $\tau$ be the inverse map of the map $g \mapsto g\left(x_{0}\right)$ from $T$ to $\Omega$. Then the map

$$
\widetilde{Z} \longrightarrow T \times Z_{x_{0}},(x, y) \mapsto\left(\tau(x), \tau(x)^{-1}(y)\right),
$$

is an isomorphism. So $Z_{x_{0}}$ is irreducible. 
iii) Let $X$ be an irreducible component of $Y_{x_{0}}$. Then $\left(\left\{x_{0}\right\} \times X\right)^{\Phi}$ is irreducible as the closure of the image of an irreducible variety by a regular map. If $Z$ is an irreducible component of $Y$ which contains $\left(\left\{x_{0}\right\} \times X\right)^{\Phi}$ then $Z_{x_{0}}$ contains $X$. So by (ii), $X$ is equal to $Z_{x_{0}}$ and $Z$ is equal to $\left(\left\{x_{0}\right\} \times X\right)^{\Phi}$ since $\Phi\left(G \times\left\{x_{0}\right\} \times X\right)$ is open in $Z$. We then deduce that the map $Z \mapsto Z_{x_{0}}$ is the inverse of the map $X \mapsto\left(\left\{x_{0}\right\} \times X\right)^{\Phi}$.

Let us recall that $\mathbf{B}_{-}$is the normalizer in $G$ of the Borel subalgebra $\mathfrak{b}_{-}=\mathfrak{h} \oplus \mathfrak{u}_{-}$.

Lemma 47. - Let $\mathbf{U}$ be the unipotent radical of the normalizer of $\mathfrak{b}$ in $G$. Then there exists a closed subset $Z$ of $\mathbf{U}$ such that the map

$$
\mathbf{B}_{-} \times Z \rightarrow \text { G.e },(g, k) \mapsto g k(e),
$$

is an isomorphism onto an open subset $V$ of G.e containing e.

Proof. - As $\mathbf{B}_{-} \mathbf{U}$ is an open subset of $G$, its image by the map $g \mapsto g(e)$ from $G$ to $G$.e is an open subset $V$ containing $e$. Moreover, the map

$$
\mathbf{B}_{-} \times \mathbf{U} . e \rightarrow V,(g, x) \mapsto g(x)
$$

is an isomorphism since $\mathbf{U}$ contains the stabilizer of $e$ in $G$ and $\mathbf{B}_{-} \cap \mathbf{U}=\left\{\mathbf{1}_{\mathfrak{g}}\right\}$. By $[\mathbf{P u}$ (Ch. I, Part. II, §3), there exists a complement $\mathfrak{m}$ of $\mathfrak{g}(e)$ in $\mathfrak{u}$ and a basis $v_{1}, \ldots, v_{m}$ of $\mathfrak{m}$ such that the map

$$
\mathbb{C}^{m} \rightarrow \text { U.e },\left(t_{1}, \ldots, t_{m}\right) \mapsto \exp \left(t_{1} \operatorname{ad} v_{1}\right) \cdots \exp \left(t_{m} \operatorname{ad} v_{m}\right)(e)
$$

is an isomorphism. Then the map

$$
\mathbb{C}^{m} \rightarrow \mathbf{U},\left(t_{1}, \ldots, t_{m}\right) \mapsto \exp \left(t_{1} \operatorname{ad} v_{1}\right) \cdots \exp \left(t_{m} \operatorname{ad} v_{m}\right)
$$

is proper and its image $Z$ is closed in $\mathbf{U}$. Moreover, the map

$$
\mathbf{B}_{-} \times Z \rightarrow V,(g, k) \mapsto g k(e)
$$

is an isomorphism.

Let $\mathcal{N}_{\mathfrak{g}, e}$ be the subset of elements $y$ of $\mathfrak{g}$ such that $(e, y)$ is in $\mathcal{N}_{\mathfrak{g}}$. From Theorem 1, Lemma 46, (iii) and Lemma 47, we deduce the following result:

Corollary 48. - The map $X \mapsto(\{e\} \times X)^{\Phi}$ is a bijection from the set of irreducible components of $\mathcal{N}_{\mathfrak{g}, e}$ onto the set of irreducible components of $\mathcal{N}_{\mathfrak{g}}$.

According to Section $2,8, \mathcal{N}_{\mathfrak{g}, e}$ has a natural structure of scheme. The following result is also a corollary of Theorem 1 .

Corollary 49. - The subset $\mathcal{N}_{\mathfrak{g}, e}$ is a complete intersection of dimension $\mathrm{b}_{\mathfrak{g}}-\mathrm{rkg}$. 
Proof. - As $\mathcal{N}_{\mathfrak{g}, e}$ is the nullvariety in $\mathfrak{g}$ of $b_{\mathfrak{g}}$ polynomial functions, the dimension of any irreducible component of $\mathcal{N}_{\mathfrak{g}, e}$ is at least $\mathrm{b}_{\mathfrak{g}}-$ rk $\mathfrak{g}$. Let $Y$ be an irreducible component of $\mathcal{N}_{\mathfrak{g}}$. By Theorem 1, since G.e is an open dense subset of $\mathfrak{N}_{\mathfrak{g}}, Y_{e}$ has dimension $\mathrm{b}_{\mathfrak{g}}-\mathrm{rk} \mathfrak{g}$. On the other hand, by Corollary 48 , $Y_{e}$ is an irreducible component of $\mathcal{N}_{\mathfrak{g}, e}$ and any irreducible component of $\mathcal{N}_{\mathfrak{g}, e}$ is obtained in this way, whence the corollary.

Let $\mathfrak{p}=\mathfrak{l}_{\mathfrak{p}} \oplus \mathfrak{u}_{\mathfrak{p}}$ be a parabolic subalgebra of $\mathfrak{g}$ containing $\mathfrak{b}$, where $\mathfrak{l}_{\mathfrak{p}}$ is the $\mathfrak{h}$-stable Levi subalgebra of $\mathfrak{p}$ and where $\mathfrak{u}_{\mathfrak{p}}$ is the nilpotent radical of $\mathfrak{p}$. We denote by $\varpi_{\mathfrak{l}_{\mathfrak{p}}}$ and $\varpi_{\mathfrak{u}_{\mathfrak{p}}}$ the projections from $\mathfrak{p}$ to $\mathfrak{l}_{\mathfrak{p}}$ and $\mathfrak{u}_{\mathfrak{p}}$ respectively.

Lemma 50. - The map $Y \mapsto Y+\mathfrak{u}_{\mathfrak{p}}$ is a one-to-one correspondence between the set of irreducible components of $\mathcal{N}_{\mathfrak{l}_{\mathfrak{p}}, \varpi_{\mathfrak{p}}(e)}$ and the set of irreducible components of $\mathcal{N}_{\mathfrak{g}, e}$ contained in $\mathfrak{p}$.

Proof. - Let $Y$ be an irreducible component of $\mathcal{N}_{\mathfrak{l}_{\mathfrak{p}}, \varpi_{\mathfrak{l}}(e)}$. Then the set $Y+\mathfrak{u}_{\mathfrak{p}}$ is contained in the intersection of $\mathcal{N}_{\mathfrak{g}, e}$ and $\mathfrak{p}$. As $e$ is a regular nilpotent element of $\mathfrak{g}, \varpi_{\mathfrak{l}_{\mathfrak{p}}}(e)$ is a regular nilpotent element of $\mathfrak{l}_{\mathfrak{p}}$. So by Corollary 49, $Y$ has dimension $\mathrm{b}_{\mathfrak{l}_{\mathfrak{p}}}-$ rkg. Then $Y+\mathfrak{u}_{\mathfrak{p}}$ has dimension

$$
b_{\mathfrak{l}_{\mathfrak{p}}}-\operatorname{rkg}+\operatorname{dim} \mathfrak{u}_{\mathfrak{p}}=b_{\mathfrak{g}}-\operatorname{rkg} .
$$

According to Corollary 49, we deduce that $Y+\mathfrak{u}_{\mathfrak{p}}$ is an irreducible component of $\mathcal{N}_{\mathfrak{g}, e}$. Thus, the above map is well-defined. It is clearly injective. Prove now that it is surjective.

Let $Z$ be an irreducible component of $\mathcal{N}_{\mathfrak{g}, e}$ contained in $\mathfrak{p}$. Then $\varpi_{\mathfrak{l}_{\mathfrak{p}}}(Z)$ is contained in an irreducible component of $\mathcal{N}_{\mathfrak{l}_{\mathfrak{g}}, \varpi_{\mathfrak{p}}(e)}$. Let $Y$ be such an irreducible component. Then $Y+\mathfrak{u}_{\mathfrak{p}}$ is an irreducible subset of $\mathcal{N}_{\mathfrak{g}, e}$ which contains $Z$. So, $Z$ is equal to $Y+\mathfrak{u}_{\mathfrak{p}}$.

Let $N_{\mathfrak{g}}$ be the set of irreducible components of $\mathcal{N}_{\mathfrak{g}, e}$. We denote by $\Upsilon_{\mathfrak{g}}$ the set of proper parabolic subalgebras strictly containing $\mathfrak{b}$. Let $N_{\mathfrak{g}}^{\prime}$ be the subset of irreducible components of $\mathcal{N}_{\mathfrak{g}, e}$ which are not contained in any element of $\Upsilon_{\mathfrak{g}}$.

Lemma 51. - If $\operatorname{dim} \mathfrak{g}=3$, then $\left|N_{\mathfrak{g}}^{\prime}\right|=0$. Otherwise, $\left|N_{\mathfrak{g}}^{\prime}\right| \geq 1$.

Proof. - The set $\Upsilon_{\mathfrak{g}}$ is nonempty if and only if $\operatorname{dim} \mathfrak{g}>3$. Suppose now $\operatorname{dim} \mathfrak{g}>3$. Let $\alpha^{\#}$ be the highest root of $\mathcal{R}_{+}$. By Lemma 42, $\mathcal{N}_{\mathfrak{g}, e}$ contains $\left[e, \mathfrak{g}^{-\alpha^{\#}}\right]$. So it suffices to prove that $\left[e, \mathfrak{g}^{-\alpha^{\#}}\right]$ is not contained in $\mathfrak{p}$ for any $\mathfrak{p}$ in $\Upsilon_{\mathfrak{g}}$. For $\alpha$ in $\mathcal{R}$, we fix a nonzero element $x_{\alpha}$ in $\mathfrak{g}^{\alpha}$. The elements $x_{\alpha}$ may be chosen so that $e=\sum_{\alpha \in \Pi} x_{\alpha}$. Suppose that there is $\mathfrak{p}$ in $\Upsilon_{\mathfrak{g}}$ such that $\left[x_{-\alpha^{\#}}, e\right]$ belongs to $\mathfrak{p}$. We can suppose that $\mathfrak{p}$ is a maximal element of $\Upsilon_{\mathfrak{g}}$ corresponding to a simple root $\beta$. A short discussion shows that $\beta$ satisfies the two following conditions: 
1) for all $\alpha$ in $\Pi \backslash\{\beta\}, \alpha^{\#}-\alpha$ is not a root,

2) $\alpha^{\#}-\beta$ is a root and $n_{\beta}=1$, where $n_{\beta}$ is the coordinate at $\beta$ of $\alpha^{\#}$ in the basis $\Pi$.

A quick look on the classification of root systems shows up that there is no simple root satisfying both Conditions (1) and (2). As we obtain a contradiction, the result follows.

Proposition 52. - The number of irreducible components of $\mathcal{N}_{\mathfrak{g}}$ is equal to $\left|N_{\mathfrak{g}}\right|$ and $\left|N_{\mathfrak{g}}\right|$ satisfies the following recursive relation:

$$
\left|N_{\mathfrak{g}}\right|=\left|N_{\mathfrak{g}}^{\prime}\right|+\left(\sum_{\mathfrak{p} \in \Upsilon_{\mathfrak{g}}} \prod_{\substack{\mathfrak{l} \text { simple factor } \\ \text { of }\left[\mathfrak{l}, \mathfrak{l}_{\mathfrak{p}}\right]}}\left|N_{\mathfrak{l}}^{\prime}\right|\right)+1 .
$$

Proof. - By Corollary 48, the number of irreducible components of $\mathcal{N}_{\mathfrak{g}}$ is $\left|N_{\mathfrak{g}}\right|$. If $\operatorname{dim} \mathfrak{g}=3$, then $\mathcal{N}_{\mathfrak{g}, e}$ is the line generated by $e, \Upsilon_{\mathfrak{g}}=\emptyset,\left|N_{\mathfrak{g}}^{\prime}\right|=0$ and $\left|N_{\mathfrak{g}}\right|=1$, whence the expected relation. Suppose now $\operatorname{dim} \mathfrak{g}>3$. We can write

$$
N_{\mathfrak{g}}=N_{\mathfrak{g}}^{\prime} \cup\left(\bigcup_{\mathfrak{p} \in \Upsilon_{\mathfrak{g}}} N_{\mathfrak{g}}^{(\mathfrak{p})}\right) \cup\{\mathfrak{u}\}
$$

where, for $\mathfrak{p}$ in $\Upsilon_{\mathfrak{g}}, N_{\mathfrak{g}}^{(\mathfrak{p})}$ is the set of irreducible components of $\mathcal{N}_{\mathfrak{g}, e}$ contained in $\mathfrak{p}$ and not contained in any element of $\Upsilon_{\mathfrak{g}}$ strictly contained in $\mathfrak{p}$. Thus the terms of the above union are pairwise disjoint and we have

$$
\left|N_{\mathfrak{g}}\right|=\left|N_{\mathfrak{g}}^{\prime}\right|+\sum_{\mathfrak{p} \in \Upsilon_{\mathfrak{g}}}\left|N_{\mathfrak{g}}^{(\mathfrak{p})}\right|+1 .
$$

It remains to prove the equality

$$
\left|N_{\mathfrak{g}}^{(\mathfrak{p})}\right|=\prod_{\substack{\mathfrak{l} \text { simple factor } \\ \text { of }\left[\mathfrak{l}_{\mathfrak{p}}, \mathfrak{l}\right]}}\left|N_{\mathfrak{l}}^{\prime}\right|,
$$

for any $\mathfrak{p}$ in $\Upsilon_{\mathfrak{g}}$. Let $\mathfrak{p}$ be in $\Upsilon_{\mathfrak{g}}$. By Lemma 50, the map $Y \mapsto Y+\mathfrak{u}_{\mathfrak{p}}$ is a bijection from $N_{\mathfrak{l}_{\mathfrak{p}}}$ to the subset of irreducible components of $\mathcal{N}_{\mathfrak{g}, e}$ contained in $\mathfrak{p}$. Moreover, $Y+\mathfrak{u}_{\mathfrak{p}}$ belongs to $N_{\mathfrak{g}}^{(\mathfrak{p})}$ if and only if $Y$ belongs to $N_{\mathfrak{l}_{\mathfrak{p}}}^{\prime}$. So $\left|N_{\mathfrak{l}_{\mathfrak{p}}}^{\prime}\right|=\left|N_{\mathfrak{g}}^{(\mathfrak{p})}\right|$. Let $\mathfrak{l}_{1}, \ldots, \mathfrak{l}_{m}$ be the simple factors of $\mathfrak{l}_{\mathfrak{p}}$ and let $Y$ be in $N_{\mathfrak{l}_{\mathfrak{p}}}$. As remarked in Subsection [3, $Y=Y_{1} \times \cdots \times Y_{m}$ where $Y_{i}$ is an irreducible component of $\mathcal{N}_{\mathfrak{l}_{i}, e_{i}}$, if $e_{i}$ is the component of $\varpi_{\mathfrak{l}_{\mathfrak{p}}}(e)$ on $\mathfrak{l}_{i}$, for $i=1, \ldots, m$. Then, $Y \in N_{\mathfrak{l}_{\mathrm{p}}}^{\prime}$ if and only if, for any $i \in\{1, \ldots, m\}, Y_{i}$ is not contained in 
any element of $\Upsilon_{\mathfrak{l}_{i}}$, that is $Y_{i} \in N_{\mathfrak{l}_{\mathfrak{p}}}^{\prime}$. Hence

$$
\left|N_{\mathfrak{l}_{\mathfrak{p}}}^{\prime}\right|=\prod_{i=1}^{m}\left|N_{\mathfrak{l}_{i}}^{\prime}\right|,
$$

and the proposition follows, by Relation (12).

Remark 9. - Considering the formula established in Proposition 52, we see that the number $\left|N_{\mathfrak{g}}\right|$ becomes considerably big while the dimension of $\mathfrak{g}$ grows up. For example we have:

$$
\left|N_{\mathfrak{S l}_{2}}\right|=1,\left|N_{\mathfrak{S l}_{3}}\right| \geq 2,\left|N_{\mathfrak{s l}_{4}}\right| \geq 4,\left|N_{\mathfrak{s l}_{5}}\right| \geq 7,\left|N_{\mathfrak{s l}_{6}}\right| \geq 12, \ldots .
$$

\section{References}

[Ba] V. V. Batyrev, Stringy Hodge numbers of varieties with Gorenstein canonical singularities, Integrable systems and algebraic geometry (Kobe/Kyoto), World Scientific Publishing Company, River Edge, New-Jersey (1998), p. 1-32.

[Bol] A. V. Bolsinov, Commutative Families of Functions Related to Consistent Poisson brackets, Acta Applicandae Mathematicae, vol. 24 (1991), nº 1, p. 253-274.

[Bou] N. Bourbaki, Lie groups and Lie algebras. Chapters 4-6. Translated from the 1968 French original by Andrew Pressley, Springer-Verlag, Berlin (2002).

[Br] W. Bruns and J. Herzog, Cohen-Macaulay rings, Cambridge studies in advanced mathematics $\mathbf{n}^{\circ} 39$ (1996), Cambridge University Press, Cambridge.

[Ch] J-Y. Charbonnel, Complexe canonique de deuxième espèce, variété commutante et bicône nilpotent d'une algèbre de Lie réductive, arXiv:math.RT/0509272.

[Cr] A. Craw, An introduction to motivic integration, American Mathematical Society, vol. 3 (2004), p. 203-225.

[DL1] J. Denef and F. Loeser, Motivic Igusa zeta function, Journal of Algebraic Geometry, vol. 7, $\mathbf{n}^{\circ} \mathbf{3}$ (1998), p. 505-537.

[DL2] J. Denef and F. Loeser, Germs of arcs on singular algebraic varieties and motivic integration, Inventiones Mathematicae, vol. 135 (1999), p. 201-232.

[DGT] D. Deturck, H. Goldschmidt, J. Talvacchia, Connections with prescribed curvature and Yang-Mills Currents: the semi-simple case, Annales Scientifiques de l'École Normale Supérieure t. 24 (1991), p. 57-112.

[Di] J. Dixmier, Champs de vecteurs adjoints sur les groupes et algèbres de Lie semisimples, Journal für die reine und angewandte Mathematik, Band. 309 (1979), p. 183-190.

[El1] R. Elkik, Singularités rationnelles et déformations, Inventiones Mathematicae $\mathbf{n}^{\circ} \mathbf{4 7}$ (1978), p. 139-147.

[El2] R. Elkik, Rationalité des singularités canoniques, Inventiones Mathematicae $\mathbf{n}^{\circ} 64$ (1981), p. 1-6.

[Fl] H. Flenner, Rationale quasihomogene Singularitäten, Archiv der Mathematik $\mathbf{n}^{\circ} 36$ (1981), p. 35-44. 
[GG] W. L. Gan and V. Ginzburg, Almost-commuting variety, D-modules, and Cherednik algebras, with an appendix by V. Ginzburg, International Mathematics Research Papers (2006), 26439, p. 1-54.

[Ha] R. Hartshorne, Algebraic Geometry, Graduate Texts in Mathematics $\mathbf{n}^{\circ} \mathbf{5 2}$ (1977), Springer-Verlag, Berlin Heidelberg New York.

[He] W. H. Hesselink, Cohomology and the Resolution of Nilpotent Variety, Mathematische Annalen $\mathbf{n}^{\circ} \mathbf{2 2 3}$ (1976), p. 249-252.

[Hi] H. Hironaka, Resolution of singularities of an algebraic variety over a field of characteristic zero: I, II, Annals of Mathematics, vol. 79 (1964), p. 109-326.

[Kon] M. Kontsevich, Motivic integration, Lecture at Orsay (1995).

[Kos1] B. Kostant, The principal three-dimensional subgroup and the Betti numbers of a complex simple Lie Group, American Journal of Mathematics $\mathbf{n}^{\circ} 81$ (1959), p. $973-1032$.

[Kos2] B. Kostant, Lie group representations on polynomial rings, American Journal of Mathematics $\mathbf{n}^{\circ} \mathbf{8 5}$ (1963), p. 327-404.

[KrW1] H. Kraft and N. Wallach, On the Nullcone of Representation of Reductive Groups, Pacific Journal of Mathematics, vol. 224 (2006), p. 119-140.

[KrW2] H. Kraft and N. Wallach, Polarizations and nullcone of representations of reductive groups, http://math.ucsd.edu/ nwallach/KWpolarnullcone.pdf (2007).

[Kr] H. Kraft, Geometrische Methoden in der Invariantentheorie, Aspekte der Mathematik, vol. D1, Vieweg Verlag, Braunschweig/Wiesbaden (1985), (2., durchgesehene Auflage).

[LS] T. Levasseur and J. T. Stafford, The Kernel of an Homomorphism of HarishChandra, Annales Scientifiques de l'École Normale Supérieure t. 29 (1996), p. 385397.

[Lo] E. Looijenga, Motivic measures, Séminaire Bourbaki, Astérisque $\mathbf{n}^{\circ} \mathbf{2 7 6}$ (1999/2000), p.267-297.

[Ma] H. Matsumura, Commutative ring theory, Cambridge studies in advanced mathematics $\mathbf{n}^{\circ} \mathbf{8}$ (1986), Cambridge University Press, Cambridge, London, New York, New Rochelle, Melbourne, Sydney.

[Mu] M. Mustaţă, Jet schemes of locally complete intersection canonical singularities, with an appendix by David Eisenbud and Edward Frenkel, Inventiones Mathematicae, vol. 145 (2001), p. 397-424.

[PYa] D. I. Panyushev and O. Yakimova, The Argument Shift Method and Maximal Commutative Subalgebras of Poisson Algebras, Mathematical Research Letters, vol. 15, $\mathbf{n}^{\circ} \mathbf{2}$ (2008), p. 239-249.

[LMP] M. Losik, P. W. Michor and V. Popov, Polarizations in Invariant Theory, Journal of Algebra, vol. 301 (2006), p. 406-424.

[Po] V. Popov, The Cone of Hilbert Nullforms, Steklov Institute of Mathematics 241 (2003), p. 177-194.

[Pu] L. Pukanszky, Leçons sur les représentations des groupes, Monograhie de la Société Mathématique de France $\mathbf{n}^{\circ} \mathbf{2}$ (1967), Dunod, Paris.

[Ri1] R. W. Richardson, Commuting Varieties of Semisimple Lie Algebras and Algebraic Groups, Compositio Mathematica, vol. 38 (1979), p. 311-322. 
[Ri2] R. W. Richardson, Normality of G-stable subvariaties of a semisimple Lie algebra, Lecture Notes in Mathematics, vol. 1271, (1987), p. 243-264.

[Ri3] R. W. Richardson, Derivatives of invariant polynomials on a semisimple Lie algebra in "Microconference on harmonic analysis and operator algebras", Proceedings of the Centre of Mathematical Analysis, Australian National University 15, Australian National University, Camberra (1987), p. 228-241.

[Ri4] R. W. Richardson, Conjugacy classes of $n$-tuples in Lie algebras and algebraic groups, Duke Mathematical Journal, vol. 57, $\mathbf{n}^{\circ} \mathbf{1}$ (1988), p. 1-35.

[Ri5] R. W. Richardson, Irreducible Components of the Nullcone, in "Invariant Theory" (Denton, TX, 1986), Contemporary Mathematics (88) (1989), American Mathematical Society, Providence, Rhode Island.

[TYu] P. Tauvel and R. W. T. Yu, Lie Algebras and Algebraic groups, Monographs in Mathematics (2005), Springer, Berlin Heidelberg New York.

[Ve] F. D. Veldkamp, The center of the universal enveloping algebra of a Lie algebra in characteristic $p$, Annales Scientifiques de L'École Normale Supérieure $\mathbf{n}^{\circ} \mathbf{5}(1972)$ , p. $217-240$.

J-Y Charbonnel, Université Paris 7 - CNRS, Institut de Mathématiques de Jussieu, Théorie des groupes, Case 7012, 2 Place Jussieu, 75251 Paris Cedex 05, France E-mail : jyc@math.jussieu.fr

A. Moreau, ETH Zürich, Departement Mathematik, HG G66.4, Rämistrasse 101, 8092 Zürich, Switzerland • E-mail : anne.moreau@math.ethz.ch 